\title{
25. Kongress des Medizinisch-Wissenschaftlichen Beirates der Deutschen Gesellschaft für Muskelkranke (DGM) e. V.
}

Datum/Ort:

24.-27. März 2021, Digital
Tagungspräsident:

PD Dr. med. Julian Grosskreutz (Jena)

\section{Vorträge}

Gesellschaftlicher Impact - Eintritt in das Zeitalter der

Gentherapie

\section{2 Hereditäre ALS zwischen Ethik und Genetik}

Autoren Knoepffler $\mathrm{N}^{1}$

Institute 1 Institut für Geschichte, Theorie und Ethik der Medizin, Bereich Ethik in den Wissenschaften, Jena, Deutschland

DOI 10.1055/s-0041-1723646

Im Unterschied zu sporadischen Formen der ALS kann bei bestimmten, genetisch bedingten Erkrankungen die Kausalität sehr gut beschrieben werden. Im Vortrag stelle ich die Familiengeschichte einer autosomal dominanten Form der ALS kurz vor und erläutere die ethische Relevanz diagnostischer und therapeutischer Herausforderungen. Dabei wird auf die Bedeutung der Möglichkeit der Präimplantationsdiagnostik für Nachkommen von Trägern der Erbveränderung eingegangen, die nach dem Urteil von entsprechenden Ethikkommissionen rechtlich zulässig wäre, aber gesellschaftlich umstritten ist. In dem Beitrag wird aus ethischer Sicht dafür argumentiert, dass auch eine sich später im Leben manifestierende, schwerwiegende Erkrankung ein hinreichender Grund für den Einsatz dieser Diagnostik ist. In einem weiteren Schritt wird auf die Frage eingegangen, wie mit dem Wunsch von ALS-Patienten nach Sterbehilfe umzugehen ist, zumal bei hereditären Formen die prognostische Sicherheit sehr groß ist, also aufgrund familiärer Erfahrung die vermutlichen nächsten Einschränkungen antizipiert werden können. Hier wird vor dem Hintergrund des Urteils des Bundesverfassungsgerichts vom 26. Februar 2020, welches das Recht auf einen selbstbestimmten Tod verteidigte, dafür argumentiert, dass eine Hilfe zur Selbsttötung gerade keine Aufgabe von Angehörigen sein darf, sondern die Aufgabe einer Ärztin oder eines Arztes des Vertrauens ist, sofern er bzw. sie eine solche Handlung vor ihrem Gewissen vertreten können.

\section{Molekulare Mechanismen der Myositis und Myasthenie}

\section{7 Immunmodulatorische Funktion von GM-CSF in idiopathisch inflammatorischen Myopathien}

Autoren Cengiz D ${ }^{1}$, Schubert J ${ }^{1}$, Nelke $C^{1}$, SG Meuth ${ }^{2}$, Müntefering $\mathrm{T}^{2,1}$, Ruck $\mathrm{T}^{2,1}$

Institutes 1 Klinik für Neurologie mit Institut für Translationale Neurologie, Universitätsklinikum, Münster, Deutschland; 2 Klinik für Neurologie, Universitätsklinikum, Düsseldorf, Deutschland DOI 10.1055/s-0041-1723647

Einleitung Idiopathische inflammatorische Myopathien (IIM) sind entzündliche Erkrankungen der Skelettmuskulatur. Die Pathogenese wird durch das Zusammenspiel von Skelettmuskel-, Muskelendothel- und Immunzellen getragen. Zytokine spielen hierbei in der Steuerung dieser Interaktionen eine zentrale Rolle. Das Zytokin GM-CSF ist maßgeblich an der Entstehung diverser
Autoimmunerkrankungen beteiligt und wird zu deren Therapie aktuell in klinischen Studien erforscht. Die Rolle von GM-CSF für die inflammatorischen Prozesse der IIMs ist allerdings weitestgehend unbekannt und deren Untersuchung ist daher Ziel dieser Arbeit.

Methoden Humane und murine Skelettmuskel- und Endothelzellen wurden mit GM-CSF und anderen pro- und anti-inflammatorischen Zytokinen stimuliert und Genexpressions- und Durchflusszytometrie-Analysen durchgeführt. Weiterhin wurde die immunologische Relevanz von GM-CSF auf die Immunzellmigration untersucht.

Ergebnisse Die Stimulation mit pro- und anti-inflammatorischen Zytokinen reguliert die Expression von GM-CSF und des GM-CSF Rezeptors von Muskelund Endothelzellen. Funktionelle Untersuchungen zeigten zudem, dass GM-CSF zu einer erhöhten Expression von Adhäsions- und kostimulatorischen Molekülen, sowie zur erhöhten Immunzellmigration über Muskelendothelbarrieren führt. Diskussion Der GM-CSF Signalweg beeinflusst die zelluläre Interaktion von Muskel-, Endothel- und Immunzellen im Skelettmuskel und könnte damit als therapeutisches Ziel in den IIMs genutzt werden.

Molekulare Schlüsselwege und Motoneuronerkrankungen

\section{9 Das Zytoskelett von Axonen - ein funktionales Multitalent}

Autoren Brill MS ${ }^{1}$

Institute 1 Technische Universität München, Institut für Zellbiologie des Nervensystems, München, Deutschland

DOI 10.1055/s-0041-1723648

Neurone sind Zellen, die über Fortsätze (Axone) verfügen, die elektrische Signale leiten. Axone enden in Synapsen, an denen die Erregung mittels Neurotransmitter den Kontakt zu anderen Zellen oder Geweben ermöglichen. Motoneurone ermöglichen so die willentliche Kontraktion von Muskeln. Axone können erstaunlich lang werden, im Menschen bis zu einem Meter, während die Zellkörper um ein vielfaches kleiner sind (ca. 50 - $100 \mu \mathrm{m}$ ).

Das Zytoskelett dient dem Neuron als strukturerhaltendes System und ist essentiell für die Homöostase. Das axonale Caliber wird von Neurofilamenten reguliert, Mikrotubuli, röhrenartige dynamische Strukturen ermöglichen den Transport von Organellen, die hauptsächlich im Soma produziert werden. Besonders Neurone mit langen Fortsätzen - z. B. Motoneurone und sensorische Neurone - stellt das vor logistische Herausforderungen. Wie stellt ein Axon z. B. sicher, dass alle Organellen gleich verteilt werden? Mikrotubuli sind außerdem dynamische Strukturen, die am plus-Ende wachsen oder schrumpfen können, von Mikrotubuli-schneidenden Enzymen geschnitten werden und mit post-translationalen Modifikationen dekoriert sind. Wie diese Modifikationen die Funktion von Mikrotubuli beeinflussen, bzw. was Strukturveränderungen an Mikrotubuli auf axonaler, zellbiologischer Ebene bewirken, ist noch unverstanden. Diesen Fragen gehen wir in transgenen und knock-out Mausmodellen nach, in denen wir Motoreneurone mit Hilfe von fluoreszenten Sensoren "live" mikroskopieren. 


\section{10 Amyotrophic lateral sclerosis patients show increased peripheral and intrathecal $\mathrm{T}$ cell activation}

Authors Pawlitzki $\mathrm{M}^{1}$, Schulte-Mecklenbeck $A^{1}$, Schreiber $S^{2}$, Vielhaber $S^{2}$, Herty $\mathrm{M}^{3}$, Marten $\mathrm{A}^{1}$, Pfeuffer $\mathrm{S}^{1}$, Wiendl $\mathrm{H}^{1}$, CC Gross ${ }^{1}$, SG Meuth ${ }^{1,4}$, Boentert $M^{1}$, Rolfes $L^{1}$

Institute 1 Klinik für Neurologie mit Institut für translationale Neurologie Münster, Klinik für Neurologie, Münster, Deutschland; 2 Otto-von-Guericke Universität, Klinik für Neurologie, Magdeburg, Deutschland; 3 RWTH Aachen, Mathematik, Aachen, Deutschland; 4 Heinrich Heine Universität, Klinik für Neurologie, Düsseldorf, Deutschland

DOI 10.1055/s-0041-1723649

Objective We investigated intrathecal and peripheral immune profiles in amyotrophic lateral sclerosis (ALS) patients compared to controls free of neurological disorders (CTRL) and patients with dementia (DEM) or primary progressive multiple sclerosis (PPMS) to elucidate whether compartment-specific inflammation contributes to ALS pathophysiology.

Methods Conventional cerebrospinal fluid (CSF) parameters were examined in 308 patients including 132 ALS patients. In a subgroup of 41 ALS patients, extensive flow-cytometric immune cell profiling in peripheral blood (PB) and CSF was performed and compared with data from 26 CTRL, 57 DEM and 27 PPMS patients.

Results ALS patients presented with significantly increased frequencies of CD4 + and CD8+ T cells expressing the activation marker HLA-DR in PB and CSF compared to CTRL. The increased intrathecal levels of T-cell activation were comparable to DEM and PPMS. Moreover, peripheral CD4+ and CD8+ T cell HLA-DR expression significantly exceeded DEM and PPMS. While T-cell activation did not correlate with clinical measures, patients with high disease disability and rapid progression showed reduced intrathecal levels of immuneregulatory CD56bright natural killer (NK) cells.

Conclusion Increased peripheral and intrathecal activation of CD4+ and CD8+ $\mathrm{T}$ cells concomitant with diminished immune regulation by CD56bright NK cells suggest an involvement of these immune cells in ALS pathophysiology.

\section{Bildgebung und Krankheitsprogression}

\section{19 The value of qualitative muscle MRI in the diagnostic procedures of myopathies: a biopsy- controlled study in 191 patients}

Authors Urban $D^{1}$, Mohamed $M^{2}$, AC Ludolph ${ }^{1}$, Kassubek J ${ }^{1}$, Rosenbohm $A^{1}$ Institute 1 Universität Ulm, Neurologie, Ulm, Deutschland; 2 UNiversitäts- und Rehabilitationskliniken Ulm, Neuroradiologie, Ulm, Deutschland DOI 10.1055/s-0041-1723650

Background The role of muscle MRI in the diagnostic procedures of myopathies is still controversially discussed. The current study was designed to analyze the status of muscle MRI, electromyography (EMG), and muscle biopsy in different cases of clinically suspected myopathy.

Methods A total of 191 patients (male: $n=12$, female: $n=79$ ) with suspected myopathy received muscle MRI, electromyography (EMG), and muscle biopsy for diagnostic reasons. Muscle MRIs were analysed using standard rating protocols by two different raters independently.

Results Diagnostic findings according to biopsy results and genetic testing were as follow: noninflammatory myopathy: $\mathrm{n}=65$, inflammatory myopathy (myositis): $n=51$, neurogenic: $n=18$, unspecific: $n=23$, and normal: $n=34$. The majority of patients showed myopathic changes in the EMG. Edema, atrophy, muscle fatty replacement, and Gadolinium enhancement in MRI were observed across all final diagnostic groups. Only $30 \%$ of the myositis group showed CM uptake.

Discussion MRI findings could not distinguish different types of neuromuscular diagnostic groups in comparison to histopathologic/genetic diagnosis. Neither muscle edema nor gadolinium enhancement are able to secure a diagnosis of myositis. Although the current results do not support qualitative MRI results as the key to final diagnosis in myopathies, quantitative muscle MRI is a valuable tool with regard to pattern recognition and longterm follow up in selective genetic myopathies.

\section{22 Diagnostic and predictive urinary fingerprints in $5 q$ spinal muscular atrophy}

Authors Saffari $A^{1}$, Ziegler A ${ }^{1}$

Institute 1 Zentrum für Kinder- und Jugendmedizin, Universitätsklinikum

Heidelberg, Sektion für Neuropädiatrie und Stoffwechselmedizin, Heidelberg,

Deutschland

DOI 10.1055/s-0041-1723651

Introduction Spinal muscular atrophy (SMA) is a life-limiting neuromuscular disease. Novel treatments have improved clinical outcomes. Yet, the absence of reliable diagnostic and prognostic biomarkers renders therapeutic decisions challenging. The aim of this proof-of-concept and feasibility study was to identify noninvasive diagnostic and prognostic urinary fingerprints in children and adolescents with $5 q$ spinal muscular atrophy (SMA).

Methods Urine samples were collected from 29 treatment-naïve SMA patients, 18 patients with Duchenne muscular dystrophy (DMD) and 444 age-matched healthy controls. Urinary metabolic profiles were established using $1 \mathrm{H}-\mathrm{NMR}$ based metabolomics.

Results Urinary spectra showed disease-specific metabolic fingerprints for SMA allowing discrimination from healthy controls, and another childhoodonset neuromuscular disease, DMD. Further analysis showed reliable separation between SMA types, allowing predictions about phenotypic severity. Interestingly, spectral fingerprints of pre-symptomatic SMA children, identified by genetic newborn screening, showed heterogenous profiles, partly converging with the SMA 1 group, suggesting the potential to detect pathophysiologic changes preceding the onset of clinical signs.

Interpretation This study provides preliminary evidence for the use of $1 \mathrm{H}$ NMR-based urinary metabolic profiling in identifying non-invasive diagnostic and prognostic fingerprints in SMA.

\section{Rehabilitation und Krankheitsverlauf}

\section{25 Urotherapie Nicht medikamentöse Therapiemöglichkeiten bei neurogenen Blasen-/ Darmfunktionsstörungen}

Autoren Beyer I

Institute $\mathbf{1}$ kbo Kinderzentrum München, Urologie/neurologische

Funktionsdiagnostik, Unterschleißheim, Deutschland

DOI 10.1055/s-0041-1723652

Einleitung Bei neurogenen Erkrankungen sind Veränderungen der Funktion von Harnblase und Darm sowie der Sexualität häufige Begleiterscheinungen. Für viele Betroffene ein schambehaftetes Tabuthema und steht meist nicht vorrangig auf dem Behandlungsplan.

Dabei ist es sehr wichtig, offen mit dem Thema umzugehen, denn es gibt vielfältige Behandlungsangebote.

Material/Methoden Erstellen von individuellen Behandlungsplänen

Harnblasen-und Darmmanagement

Ergebnisse Bei neurogenen Erkrankungen stehen meist Harnblasen-und Darmfunktionstörungen nicht vorrangig auf dem Behandlungsplan, im Kontrast dazu steht jedoch die persönliche Wahrnehmung der Inkontinenz als eine erhebliche Beeinträchtigung im Alltag. Aufgrund der engen Zusammenarbeit als Urotherapeut mit dem Patienten können wertvolle Informationen entdeckt und kommuniziert werden.

Die Urotherapie bietet eine Möglichkeit, beratend und schulend zu begleiten, frühzeitig Sekundärschäden zu vermeiden und in der Kommunikation mit dem Patienten therapeutisch einen individuellen Behandlungsplan aufzustellen. Diskussion Wie sind individuelle Behandlungsansätze im darstellbar? 
Literaturverzeichnis

Hayder-Beichel D.(Hrsg.), 2013: Interdisziplinäre Kontinenzberatung. Stuttgart: Kohlhammer

Werner S. 2012: Kontinenzförderung, 1. Auflage 2012. Stuttgart: Kohlhammer

Beatmung, Ernährung und symptomatische Medikation

\section{28 Atemmuskeltraining bei ventilatorischer Insuffizienz der Myotonen Dystrophie Typ 1}

\author{
Autoren EM Heidsieck ${ }^{1}$, Wirner $C^{1}$, Gutschmidt $K^{1}$, Schoser $B^{1}$, \\ Wenninger $\mathrm{S}^{1}$ \\ Institute 1 Friedrich-Baur-Institut, LMU Klinikum München, Neurologie, \\ München, Deutschland \\ DOI 10.1055/s-0041-1723653
}

Einleitung Patienten mit Myotoner Dystrophie Typ 1 (DM1) leiden im Krankheitsverlauf an einer progredienten Atemmuskelschwäche in individueller Ausprägung, welche nicht selten zu einer Beatmungspflichtigkeit führt. In dieser Studie wurde erstmalig die Sicherheit und Effektivität eines regelmäßigen Atemmuskeltrainings mit einem mobilen Trainingsgerät untersucht.

Material/Methode In diese kontrollierte, randomisierte, dreiarmige Interventionsstudie wurden Patienten mit gesicherter DM1 eingeschlossen. Die Randomisierung erfolgte in eine der drei Gruppen inspiratorisches Krafttraining, Ausdauertraining oder kein Training der Atemmuskulatur. Regelmäßige Verlaufsuntersuchungen erfolgten zur Analyse der Veränderungen von Lungenfunktionsparametern, Blutgasanalyse, muskulären Funktionstests sowie Auswirkungen auf Lebensqualität und Muskelfunktion im Alltag. Eine Longitudinalanalyse zeigt den Verlauf über einen Studienzeitraum von 9 Monaten.

Ergebnisse Die bisher erhobenen Daten von 26 eingeschlossenen Patienten werden derzeit ausgewertet und erste Zwischenergebnisse werden präsentiert.

Diskussion Da es bisher keine kausale Therapie für Patienten mit DM1 gibt, sind neue Ansätze zur Symptomlinderung und Verbesserung der Lebensqualität und -partizipation unerlässlich. Diese Studie liefert relevante Erkenntnisse zur Sicherheit und Effektivität eines regelmäßigen Atemmuskeltrainings und wird in zukünftige Versorgungs- und Therapieempfehlungen für Patienten mit DM1 integriert werden.

Molekulare Signaturen genetischer muskulärer Erkrankungen

\section{30 Duchenne Muskeldystrophie - molekulare Signaturen und neue Therapien}

Autoren Walter $\mathrm{MC}^{1}$

Institute 1 Friedrich-Baur-Institut, Neurologische Klinik und Poliklinik, Klinikum der Ludwig-Maximilians-Universität München, München, Deutschland DOI 10.1055/s-0041-1723654

Durch enorme Fortschritte in der Grundlagenforschung und ein verbessertes Verständnis der Pathophysiologie haben sich in letzter Zeit vielfältige molekulare Therapieansätze für die Duchenne Muskeldystrophie ergeben; zahlreiche molekulare Therapien befinden sich in der Entwicklung. Neue Entwicklungen im Bereich der personalisierten Gentherapie zielen strategisch auf bestimmte, genetisch determinierte Subtypen der Erkrankung, basierend auf dem jeweiligen Krankheitsmechanismus und dem resultierenden Phänotyp, und setzen damit ein Beispiel für andere hereditäre Erkrankungen. Der Erkenntnisgewinn in diesem Bereich nimmt stetig zu; dennoch ist es noch ein weiter Weg, bevor diese Therapieformen Phänotyp und Pathologie und der betroffenen Patienten tatsächlich werden korrigieren können.

\section{31 Drosophila as a myotonic dystrophy model: insights into CELF family function during myofibril formation}

Authors Spletter $\mathrm{M}^{1}$, Nikonova $\mathrm{E}^{1}$

Institute $\mathbf{1}$ Ludwig-Maximilians-Universität, Physiologische Chemie, Martinsried-Planegg, Deutschland

DOI 10.1055/s-0041-1723655

Introduction To understand how CELF1 misregulation contributes to myotonic dystrophy (DM1), it is important to examine how RNA processing functions in healthy muscle. As sarcomere structure, muscle protein composition and many molecular aspects of CELF1 function are highly conserved, we use Drosophila melanogaster flight muscle (IFM) as a tractable genetic model. We have previously shown that the CELF1 homologue Bruno-1 (Bru1) controls flight muscle specific alternative splicing, regulating both sarcomere growth and myosin contractility.

Methods We generated new CRISPR-mediated deletions in Bru1, knocking-out all Bru1 isoforms, and performed mRNA-Seq, mass spectrometry and phenotypic analysis.

Results Stronger phenotypes in the Bru1 mutant flies revealed defects at the earliest stages of myofibril formation, notably disorganization of the actin cytoskeleton in differentiating IFM. After sarcomere formation, while thin-filament growth in length is arrested, thin-filament lateral growth is dramatically misregulated leading to formation of hollow myofibrils. In addition to sarcomeric proteins, we also identified misregulated expression of other RNA-proces sing factors.

Conclusion During muscle differentiation, Bru1 regulates cytoskeletal rearrangement necessary for myofibril formation as well as the balance in length versus lateral growth of the thin-filament. Defective RNA processing thus causes sarcomeric structural defects and malfunction of dystrophic muscle.

\section{32 Präzise Geneditierung der häufigsten LGMD2A Mutation}

Autoren Müthel $S^{1}$, Spuler $S^{1,2}$

Institutes 1 Experimental and Clinical Research Center, Berlin,

Deutschland; 2 Charite Universitätsmedizin Berlin, Berlin, Deutschland DOI 10.1055/s-0041-1723656

Die Geneditierung ist ein leistungsstarkes Werkzeug, um krankheits-auslösende Mutationen in Primärzellen von Patienten zu reparieren. In unserer Studie möchten wir Mutationen im CAPN3 Gen reparieren. Ein Verlust des davon kodierten Calpain 3 Proteins führt zu Gliedergürteldystrophie 2A (LGMD2A). Es handelt sich bei dieser progressiven Muskelerkrankung um die häufigste Gliedergürteldystrophie und bis heute gibt es keine Therapie. In unserer Kohorte befinden sich 47 LGMD2A-Patienten, die insgesamt 44 verschiedene Mutationen in CAPN3 tragen. 20 \% der Patienten haben die Mutation 550delA, was zu einer Verschiebung des offenen Leserahmens führt, wodurch es zu einer verfrühten Termination während der Bildung des Proteins kommt. Wir haben primäre Muskelstammzellen von einem homozygoten und einem heterozygoten Patienten isoliert, expandiert und die Mutation mittels CRISPR/Cas9 so repariert, dass es zu einer Wiederherstellung des offenen Leserahmens im Protein kommt. Die Reparatur erfolgt über Non-homologous end-joining, einem Mechanismus, der sowohl in sich teilenden als auch nicht-teilenden Zellen aktiv ist. Momentan analysieren wir, ob die Reparatur auch zu einer Re-Expression des Proteins führt und ob reparierte Muskelstammzellen in den Muskel eines Mausmodells integrieren können. Der hier genutzte Reparaturmechanismus bietet die Möglichkeit verschiedene Deletionen von einem Basenpaar präzise zu reparieren und hat daher hohes Potential für die Gentherapie von Muskelerkrankungen 


\section{33 Das DMDpig als translationales Großtiermodell für die Duchenne Muskeldystrophie in der präklinischen Forschung}

\author{
Autoren Donandt $\mathrm{T}^{1}$, Krause $\mathrm{S}^{1}$, Kalbe $\mathrm{C}^{2}$, LM Fonteyne ${ }^{3}$, Kessler $\mathrm{B}^{3}$, \\ Wolf $\mathrm{E}^{3}$, Walter $\mathrm{MC}^{1}$ \\ Institutes 1 Friedrich-Baur-Institut, Neurologische Klinik und Poliklinik, \\ Klinikum der Ludwig-Maximilians-Universität, München, \\ Deutschland; 2 Leibniz-Institut für Nutztierbiologie (FBN), Institut für \\ Muskelbiologie und Wachstum, Dummerstorf, Deutschland; 3 Tierärztliche \\ Fakultät, Molekulare Tierzucht und Biotechnologie, Ludwig-Maximilians- \\ Universität, München, Deutschland \\ DOI 10.1055/s-0041-1723657
}

Einleitung Das Großtiermodell für die Muskeldystrophie Duchenne (DMD) “DMDpig” ermöglicht vielversprechende Strategien in der Therapieentwicklung für DMD-Patienten. Allerdings war die erfolgreiche Differenzierung von porcinen Myoblasten in vitro lange ein Hindernis beim Nachweis von therapeutisch wiederhergestelltem Dystrophin-Protein.

Material/Methode Inzwischen wurde ein Zellkulturmodell zur Anzucht von myogenen Primärkulturen aus verschiedenen porcinen Muskeln und für die Fusion von myogenen Zellen in vitro etabliert.

Ergebnisse Es wurde ein effizientes Protokoll entwickelt, mit welchem aus Satellitenzellen eines DMDpigs sowie von DMD-Carriern und Wildtyp-Tieren unterschiedlichen Alters Myoblasten gewonnen werden können. Die Myogenität dieser Zellkulturen konnte anhand verschiedener Marker in der Immunfluoreszenz nachgewiesen werden. Ebenso gelang der Nachweis von in vitro synthetisiertem Dystrophin und weiteren myogenen Markern im Western Blot.

Diskussion Das DMDpig hat im Gegensatz zu anderen bereits etablierten Tiermodellen für die Duchenne Muskeldystrophie den Vorteil, dass der Phänotyp der erkrankten Tiere dem der menschlichen Patienten sehr ähnlich ist. Ebenso sind die DMDpigs hinsichtlich Körpergewichts und Pathophysiologie näher am Menschen, sodass eine mögliche Toxizität, andere unerwünschte Wirkungen oder die ideale Dosierung einer neuen krankheitsmodifizierenden Therapie besser antizipiert werden können.

Fallkonferenz des Referenzzentrums für Neuromuskuläre Erkrankungen

\section{34 Erstmanifestation einer sporadischen Einschlusskörperchenmyositis (sIBM) nach jahrelanger immunsuppressiver Therapie}

Autoren Winkler $\mathrm{M}^{1}$, von Landenberg $\mathrm{C}^{1}$, Kornblum $\mathrm{C}^{1}$, Reimann $\mathrm{J}^{1}$ Institute 1 Universitätsklinikum Bonn, Klinik für Neurologie, Sektion Neuromuskuläre Erkrankungen, Bonn, Deutschland

DOI 10.1055/s-0041-1723658

Zwei konkurrierende Hypothesen zur Entstehung der sIBM werden diskutiert. Eine entzündliche Initiierung, aufgrund des Angriffs zytotoxischer T-Zellen auf Muskelfasern, sowie Nachweises von Autoantikörpern, oder eine degenerative Ursache, basierend auf gestörter Autophagie, pathologischen Proteinablagerungen im Muskel und der fehlenden suffizienten Ansprache auf immunsuppressive Therapien. Eine Theorie, die beide Prozesse vereint, diskutiert eine sekundäre Degeneration ausgelöst durch einen primär entzündlichen Pathomechanismus.

Wir berichten über zwei Patienten, die unter langjährigen immunsuppressiven Therapien (17 bzw. 12 Jahre vor Diagnosestellung) eine sIBM entwickelten (klinisch und pathologisch definiert nach ENMC-Kriterien 2011). Erste Symptome traten 15 bzw. 6 Jahre nach Nieren- bzw. Lebertransplantation auf. Vor den Transplantationen lagen keine Muskelbeschwerden vor. CK-Werte waren in beiden Fällen auf $<15 x$ der oberen Norm erhöht. Beide Muskelbiopsien zeigten rimmed vacuoles, anti-p62 positive Protein-Aggregate und endomysiale entzündliche Infiltrate.

Ziel der immunsuppressiven Therapien ist die Transplantat-Toleranz, sie können so selbst als Dysregulationen des Immunsystems betrachtet werden. Dass allerdings eine Jahre vor der Entwicklung von Muskelsymptomen begonnene Immunsuppression den zur sIBM führenden Prozess nicht unterdrücken kann, scheint im Widerspruch zur Auslösung eines sekundären degenerativen Prozesses durch primär entzündliche Aktivität zu stehen.

\section{35 Indikation zur Muskelbiopsie bei isolierten Myalgien ohne Paresen, normaler CK und unauffälligem EMG}

Autoren Kendzierski $\mathrm{T}^{1}$, Nägel $\mathrm{S}^{1}$, Stoltenburg-Didinger $\mathrm{G}^{1,2}$, Zierz $\mathrm{S}^{1}$ Institutes 1 Universitätsklinik der Martin-Luther-Universität Halle/Saale, Klinik f. Neurologie, Halle, Deutschland; 2 Charité Berlin, Institut für Zell und Neurobiologie, Berlin, Deutschland

DOI 10.1055/s-0041-1723659

Bei Patienten mit ausschließlichen Myalgien ohne Paresen und normaler CK und Elektromyographie kann die Ätiologie häufig nicht sicher geklärt werden. Wir präsentieren den Fall eines 48-jährigen Patienten, der sich uns mit progredienten Myalgien und einer Belastungsintoleranz in allen Extremitäten seit dem 46. Lebensjahr vorstellte. Die Familienanamnese war nicht wegweisend. Der klinische Befund war unauffällig. Laborchemisch und elektrophysiologisch waren keine pathologischen Veränderungen zu verzeichnen. Die metabolischen Belastungstests waren unauffällig. Das MRT der Skelettmuskulatur war normal. In der Muskelbiopsie fanden sich histologisch fokal im interstitiellen Bindegewebe lokalisierte Rundzellinfiltrate und eine fokale Hochregulation von HLA-ABC auf den umgebenden Muskelfasern. Die Muskelfasern waren intakt. Die Symptome waren durch eine Glukokortikoidtherapie nicht positiv beeinflussbar. Dieser Fall belegt, dass bei Patienten mit Myalgien ohne Paresen, normaler CK und unauffälligem EMG eine Muskelbiopsie zur Diagnose einer interstitiellen Myositis gerechtfertigt ist.

\section{RZ-Fallkonferenz}

\section{36 Three cases of neurogenic muscular atrophy: combined with sporadic late onset nemaline myopathy (SLONM)?}

Authors Weis $J^{1}$, Schröder $R^{2}$, Eigler $V^{3}$, Rebel $M^{4}$, Nikolin $S^{1}$, Katona $I^{1}$, Dafotakis $\mathrm{M}^{5}$, Dohrn $\mathrm{MF}^{5,6}$, Grond $\mathrm{M}^{7}$

Institute 1 RWTH Aachen University Hospital, Institute of Neuropathology, Aachen, Deutschland; 2 Erlangen University Hospital, Institute of Neuropathology, Erlangen, Deutschland; 3 Klinikum Ludwigshafen, Department of Neurology, Ludwigshafen, Deutschland; 4 Klinikum Ludwigshafen, Department of Pathology, Ludwigshafen, Deutschland; 5 RWTH Aachen University Hospital, Department of Neurology, Aachen,

Deutschland; 6 University of Miami, Miller School of Medicine, Dr. John T.

Macdonald Foundation, Department of Human Genetics and John P. Hussman Institute for Human Genomics, Miami, FL, Vereinigte Staaten; 7 Kreisklinikum Siegen, Department of Neurology, Siegen, Deutschland DOI 10.1055/s-0041-1723660

Introduction Sporadic late-onset nemaline myopathy (SLONM) is a rare, acquired, progressive, late-onset muscle disorder. Nemaline rods found in muscle fibers are a defining feature. Clinically, SLONM is characterized by proximal muscle weakness and atrophy [1].

Cases We report three cases encountered by us during a period of eight months. A 61 year old patient presented with progressive proximal and paraspinal muscle weakness and atrophy that had started at age 58. Electrophysiology showed a mixed myopathic and neurogenic pattern. Muscle biopsy revealed prominent structural myopathy; electron microscopy (EM) showed 
numerous cytoplasmic and nuclear nemaline bodies as well as cores and other signs of myofibrillar disintegration. By serum analysis, monoclonal gammopathy type IgG Kappa was detected. Immunoglobulin treatment stopped progression and led to an improvement of symptoms. The second patient presented with progressive axial weakness, camptocormia and monoclonal gammopathy type IgG Lambda at age 56. Quadriceps femoris muscle biopsy revealed neurogenic muscular atrophy combined with electron microscopic evidence of nemaline body myopathy. Patient three presented with proximal muscle weakness. Similar to the findings in patient 2, quadriceps muscle biopsy revealed chronic neurogenic changes combined with nemaline body myopathy detected by electron microscopy. There was no evidence of a genetic disorder in any of the three patients.

Conclusions Neurogenic alterations may coincide with SLONM. As reported previously [1], intranuclear rods may be encountered in SLONM.

[1] Schnitzler LJ, Schreckenbach T, Nadaj-Pakleza A, Stenzel W, Rushing EJ, Van Damme P, Ferbert A, Petri S, Hartmann C, Bornemann A, Meisel A, Petersen JA, Tousseyn T, Thal DR, Reimann J, De Jonghe P, Martin JJ, Van den Bergh PY, Schulz JB, Weis J, Claeys KG. Sporadic late-onset nemaline myopathy: clinicopathological characteristics and review of 76 cases. Orphanet J Rare Dis. 2017;12(1):86. doi: 10.1186/s13023-017-0640-2.

\section{37 The first German patient with oculopharyngodistal myopathy?}

\author{
Authors Montagnese $\mathrm{F}^{1}$, Nishino $\mathrm{I}^{2}$, Schoser $\mathrm{B}^{1}$ \\ Institute 1 Friedrich-Baur Institut, LMU München, Neurologie, München, \\ Deutschland; 2 NCNP, Kodaira, Tokyo, Department of Neuromuscular \\ Research, Tokyo, Japan \\ DOI 10.1055/s-0041-1723661
}

Introduction Oculopharyngodistal myopathy (OPDM) is a rare hereditary myopathy mostly reported in the Asian population. Typical clinical features are: severe facial weakness, ptosis, ophtalmoparesis, dysphagia, distal muscle weakness. The muscle biopsy shows myopathic changes with rimmed vacuoles. The genetic cause of OPDM remains elusive, recent studies found repeat expansion mutations in LRP12 and GIPC1 in some OPDM patients1-2.

Case report A 33-years-old female presents since the age of 24 years progressive facial weakness, swallowing difficulties, muscle wasting and weakness of both hands. The family history is uninformative. On neurological examination the most prominent feature is the severe facial weakness with mild ptosis and horizontal ophtalmoparesis, nasal speech and dysphagia. Focal weakness and atrophies were observed at thenar eminence bilaterally. The CK, AchR-Ab, LRP4-Ab and titin-Ab were normal. The EMC showed a myopathic pattern. The repetitive nerve stimulation was normal. The muscle biopsy showed mild myopathic changes with isolated rimmed vacuoles without signs of mitochondrial pathology. Genetic analysis (PABPN, DMPK, FSHD1-2 and next generation sequencing for myopathy panel) was negative.

Discussion The clinical, EMG and histological features of this patient are highly suggestive of OPDM. The analysis of LRP12 and GIPC1 is ongoing in Tokyo. This case opens the discussion on differential diagnosis of myopathies with prominent facial weakness.

\section{38 Manifest Dystrophinopathy in a 6-years old girl}

Authors Stenzel $W^{1}$, Dittmayer $C^{1}$, HH Goebel ${ }^{1}$, von Moers $A^{1}$

Institute 1 Charité - Universitätsmedizin, Neuropathologie, Berlin, Deutschland DOI 10.1055/s-0041-1723662

Background We report on a 6-year-old girl from healthy parents with delayed motor milestones who was not able to stand up since age four years. She showed proximal weakness and a hypertrophic gastrocnemius. Cardiac function was normal, and her CK levels at admission were $>20.000 \mathrm{IU} / \mathrm{l}$.
Genetic work-up including DMD (Del/Dup, point mutation) ANO5, CAPN3, CAV3, DYSF, FKRP, GAA, MYOT, PYGM, SGCA, SGCB, SGCD, SGCG, TCAP disclosed normal findings and consequently, a muscle biopsy was performed.

We analyzed a skeletal muscle biopsy from her right quadriceps and identified fibre-type disproportion as well as single core defect. Immunohistochemistry showed absence of nNOS and a mosaic pattern of absence of the rod domain and the C-terminus of the dystrophin protein with complete absence of the $\mathrm{N}$-terminus of dystrophin. Accordingly, utrophin was strongly stained on most fibres and a-laminin 5 as well.

A diagnosis of a manifesting Duchenne phenotype of muscle affection was made, and a profound genetic workup including WES was performed, showing near complete skewed X inactivation as well as Chr. 5p and Xp translocation.

This unusual case highlights the need for muscle biopsy to identify unusual presentations and guide specific genetic workup, which would otherwise not have successfully been done.

\section{39 Klinisch-histologische Charakterisierung eines Patienten mit einer neuen Mutation im MYH14-Gen und komplexem Phänotyp aus peripherer Neuropathie, Myopathie und Heiserkeit}

Autoren Mensch $\mathrm{A}^{1}$, Weis $\mathrm{J}^{2}$, Jordan $\mathrm{B}^{1,3}$, Kendzierski $\mathrm{T}^{1}$, Schneider $\mathrm{I}^{1,4}$, Stoevesandt $D^{5}$, Stoltenburg-Didinger $G^{1}$, Kraya $T^{1,4}$, Zierz $S^{1}$, Naegel $S^{1}$ Institutes 1 Universitätsklinikum Halle (Saale), Universitätsklinik und Poliklinik für Neurologie, Halle a. d. S., Deutschland; 2 Universitätsklinikum RWTH Aachen, Institut für Neuropathologie, Aachen,

Deutschland; 3 Universitätsklinikum Heidelberg, Abteilung Neurologie und Poliklinik, Heidelberg, Deutschland; 4 Krankenhaus St. Georg, Klinik für Neurologie, Leipzig, Deutschland; 5 Universitätsklinikum Halle (Saale), Universitätsklinik und Poliklinik für Radiologie, Halle a. d. S., Deutschland DOI 10.1055/s-0041-1723663

Einleitung Mutationen im nicht-muskulären Myosin MYH14 sind mit verschiedenen Krankheitsentitäten assoziiert, unter anderem einem komplexen Phänotyp mit peripherer Neuropathie, Myopathie, Heiserkeit und Schwerhörigkeit. Seit der Erstbeschreibung in einer großen koreanischen Familie konnte der klinische Phänotyp durch Identifikation einer weiteren amerikanischen und einer kanadischen Familie detaillierter charakterisiert werden.

Methoden Wir berichten über einen deutschen Patienten mit atypischem Verlauf einer MYH14-assoziierten neuromuskulären Erkrankung aufgrund einer bisher nicht beschriebenen Mutation im MYH14-Gen.

Ergebnisse Klinisch präsentierte sich eine distale Myopathie mit früher respiratorischer Beteiligung und ausgeprägter Heiserkeit. Darüber hinaus zeigte sich eine periphere Neuropathie. Eine relevante Schwerhörigkeit bestand nicht. Die Muskelbiopsie zeigte eine vakuoläre Myopathie mit exzessiver Autophagie und Exozytose. Die Histologie des Nervus suralis ergab eine vorwiegend axonale Schädigung mit zusätzlicher demyelinisierender Läsion und geringen Zeichen einer Inflammation. Der Patient verstarb im Alter von 52 Jahren im Rahmen einer rasch progredienten respiratorischen Insuffizienz.

Zusammenfassung Der Fall erweitert das phänotypische Spektrum der MYH14-assoziierten neuromuskulären Störungen sowohl hinsichtlich des klinischen Phänotyps, des Krankheitsverlaufs als auch der histopathologischen Merkmale.

\section{40 Calpainopathie mit dem klinischen Phänotyp rezidivierender Rhabdomyolysen}

Autoren Kendzierski $T^{1}$, Mensch $A^{1}$, Nägel $S^{1}$, Stoltenburg-Didinger $G^{1,2}$, Zierz $S^{1}$

Institutes 1 Universitätsklinik der Martin-Luther-Universität Halle/Saale, Klinik f. Neurologie, Halle, Deutschland; 2 Charité Berlin, Institut für Zell und Neurobiologie, Berlin, Deutschland

DOI 10.1055/s-0041-1723664 
Calpainopathien sind phänotypisch variable hereditäre Myopathien, die sowohl autosomal- dominant als auch rezessiv vererbt werden können und die auf verschiedenen Mutationen im CAPN3-Gen beruhen. Wir präsentieren den Fall einer 58-jährigen Patientin, die sich uns mit schmerzhaften, rekurrierenden Rhabdomyolysen seit dem 46 Lebensjahr vorstellte. Die Familienanamnese war nicht wegweisend. Klinisch imponierten zwischen den Attacken eine milde persistierende Gliedergürtelschwäche und eine Belastungsintoleranz. Die CK war im Intervall bis zu 20x erhöht. Der Krankheitsverlauf war durch immunsuppressive und immunmodulatorische Medikamente nicht beeinflussbar. Die elektrophysiologischen Untersuchungen waren normal. Ein MRT der Skelettmuskulatur zeigt nur leicht Veränderungen im Caput med. des M. gastrocnemius links. In der Muskelbiopsie zeigte sich ein myopathisches Gewebssyndrom ohne arttypische Zuordnung durch umfassende immunhistochemische Reaktionen. Der Calpain-3-Westernblot zeigte eine verminderte Darstellung beider Banden (Exon 1 und 3). Die Mutation c.1063C>T im CAPN3Gen war heterozygot nachweisbar, die in den Datenbanken ClinVar und HGMD als pathogene rezessiv vererbte Variante geführt wird. Eine compound Heterozyogtie des CAPN3-Gens lag nicht vor. Der vorliegende Fall zeigt, dass rezidivierende Rhabdomyolysen möglicherweise ein Phänotyp eines Carrier-Status einer autosomal-rezessiv vererbten Calpainopathie darstellen.

\section{41 Phenotype and myopathological findings in patient with SLC18A3 mutation - does muscular biopsy help?}

Authors Della Marina $A^{1}$, Arlt $A^{2}$, Depienne $C^{3}$, Weis $\rfloor^{4}$, Gangfuß $A^{1}$, Kölbel $H^{1}$, Sickmann $A^{5}$, Freier $E^{5}$, Kohlschmidt $N^{2}$, Czech $A^{5}$, Hentschel $A^{5}$, Schara-Schmidt $U^{1}$, Roos $A^{1}$

Institute 1 Universitätsklinikum Essen, Abteilung für Neuropädiatrie, Essen, Deutschland; 2 Institut für Klinische Genetik und Tumorgenetik Bonn, Bonn, Deutschland; 3 Institut für Humangenetik, Universitätsklinikum Essen, Essen, Deutschland; 4 Institut für Neuropathologie, Uniklinik RWTH Aachen, Aachen, Deutschland; 5 Leibniz-Institut für Analytische Wissenschaften - ISAS,

Dortmund, Deutschland

DOI 10.1055/s-0041-1723665

SLC18A3 gene encodes for the vesicular Acetylcholine (Ach) transporter (VAChT) modulating the active transport of Ach at the neuromuscular junction and causing presynaptic congenital myasthenic syndrome (CMS). In VAChTdel/del mutant mice muscle biopsy (MB) showed a loss of normal myofibrillar architecture and marked atrophy. A male patient presented postpartal respiratory insufficiency with arthrogryposis multiplex congenita, muscular hypotonia, facial hypomimia, bilateral fluctuating ptosis, ophthalmoplegia and bulbar symptoms. Because of suggestive clinical phenotype for CMS, pyridostigmine was started with only partial effect. First genetical analysis revealed no pathological results, for diagnostic purpose MB was performed. It showed variabilities in fibre size as well as fibre type grouping. Electron microscopy(EM) revealed increased and enlarged lipid droplets and minicores. Lipid accumulation was confirmed by CARS(Coherent Anti-Stokes Raman Scattering) microscopy. Proteomic profiling identified protein dysregulations impacting on vulnerability of the muscle. Whole exome sequencing revealed compound heterozygosity of nonsense and missense SLC18A3 variants(c.315G>A; p.Trp105*\&.1192G>Cp. Asp398His). In our patient the phenotype was more severe compared to those determined by missense variants. Specific myopathic changes were lacking as the case in VAChTdel/del mutant mice, but lipid accumulation on EM and CARS. This findings are to be discussed in contex with literature.

\section{42 Kardiale Symptome und milde Gliedergürteldstrophie (LGMD) im Kindesalter verursacht durch eine Nonsens Mutation im BVES (c.457C >T; p. Gln153Ter)}

Autoren Schänzer $\mathrm{A}^{1}$, Brand $\mathrm{T}^{2}$, Gonzales $\mathrm{M}^{3}$, Hahn $\mathrm{A}^{4}$

Institutes 1 Institut für Neuropathologie, Justus Liebig Universität Giessen, Gießen, Deutschland; 2 Cardiac Section National Heart and Lung Institute (NHLI) Faculty of Medicine Imperial College London, London, Vereinigtes Königreich; 3 Kinderherzzentrum, JL Universität Giessen, Gießen, Deutschland; 4 Abteilung für Neuropädiatrie, JL Universität Giessen, Gießen, Deutschland DOI 10.1055/s-0041-1723666

Einleitung Genetische Mutationen, welche mit Myopathien assoziiert sind, können auch zu kardialen Symptomen führen. Bei Patienten mit Gliedergürteldystrophie (LGMD) kann eine kardiale Beteiligung in unterschiedlicher Ausprägung vorliegen.

Methoden Wir beschreiben eine Familie konsanguiner Eltern mit fünf Kindern. Drei Kinder im Alter von 7-17 Jahren (J) zeigen kardiale Symptome und erhöhte CK Werte zwischen 500-15.000 U/I. Bei allen Familienmitgliedern wurden klinische und genetische Untersuchungen durchgeführt. Eine Muskelbiospie erfolgte bei dem $17 \mathrm{~J}$ alten Patienten.

Ergebnisse Drei Kinder zeigten eine Sinusknotenerkrankung (SND) mit atrioventrikulärem Block. Nur der 17 J alte Patient zeigte zusätzlich Symptome einer milden LGMD. Die Muskelbiopsie zeigte Veränderungen im Sinne einer Muskeldystrophie. Genetische Untersuchungen (WES) identifizierten eine homozygote Nonsense-Mutation in BVES (p.Q153X (c.457>T) bei den drei erkrankten Kindern, bei den anderen Familienmitglieder war dieses Allel heterozygot. Blood vessel epicardial substance (BVES) kodiert für das Popeye domain containing 1 (POPDC1) Protein, BVES Mutationen sind assoziiert mit der seltenen LGMDR25. Ein Verlust der sarkolemmalen Lokalisation von POPDC1 und POPDC2 wurde in der Muskelbiopsie des erkrankten Patienten beobachtet.

Diskussion Bei jungen Patienten mit einer kardialen Überleitungsstörung und asymptomatischer CK Erhöhung, sollte eine BVES Mutation ausgeschlossen werden.

Stratifizierung in klinischen Studien

\section{45 Non-dystrophic myotonias - clinical and mutation spectrum of seventy German patients}

Authors Vereb $N^{1}$, Montagnese $\mathrm{F}^{1}$, Gläser $\mathrm{D}^{2}$, Gutschmidt $\mathrm{K}^{1}$, Schoser $\mathrm{B}^{1}$ Institute 1 Friedrich-Baur-Institut, München, Deutschland; 2 Genetikum, NeuUlm, Deutschland

DOI 10.1055/s-0041-1723667

Introduction Non-dystrophic myotonias (NDM) are caused by mutations in CLCN1 and SCN4A. Due to a limited number of studies, the aim was to describe the clinical and genetic spectrum of NDM in a large German cohort.

Methods We retrospectively identified all genetically confirmed NDM patients in our center and analyzed: demographics, family history, muscular features, cardiac involvement, CK, EMG, genotype, other tested genes, treatment perceived efficacy.

Results 70 patients ( 40.2 years $\pm 14.9 ; 52.8 \%$ male) were included (48 CLCN1/ 22 SCN4A). The most frequent presenting symptoms were myotonia (83.3/ $72.2 \%$ ) and myalgia (57.4/52.6\%). Except more frequent facial involvement in SCN4A and cold-sensitivity in CLCN1, no significant differences were observed. Cardiac arrhythmia or conduction defects were documented in 6 CLCN1 patients ( 3 requiring pacemaker) and 1 with SCN4A. CK was normal in $40 \%$ of patients, myotonic runs in EMG were detected in $89.1 / 78.9 \% .50 \%$ of CLCN1 patients had the classic c. 2680 C > T mutation. 12 new genetic variants are 
reported. $50 \%$ were taking anti-myotonic drugs at the last follow-up (mexiletine and lamotrigine rated best).

Discussion This study highlights the relevant clinical overlap between NDM subtypes and warrants the use of early and broad genetic investigation for the precise identification of the NDM subtype. Besides the clinical and genetic heterogeneity, the limited response to current anti-myotonic drugs constitutes a continuing challenge.

Wertigkeit von OMICS-Analysen

\section{48 NME-GPS: Gen- und Protein-Signaturen als GPS für Patienten mit neuromuskulären Erkrankungen}

Autoren Gangfuß $A^{1}$, Kölbel $H^{1}$, Della Marina $A^{1}$, Bury $G^{2}$, Kohlschmidt $N^{3}$, Arlt $A^{3}$, Nguyen $C^{4}$, Hentschel $A^{4}$, Sickmann $A^{4}$, Schara-Schmidt $U^{1}$, Roos $A^{1}$ Institutes 1 Universitätsklinikum Essen, Kinderklinik I, Neuropädiatrie, Essen, Deutschland; 2 INFORM GmbH, Aachen, Deutschland; 3 Institut für klinische Genetik und Tumorgenetik, Bonn, Deutschland; 4 Leibniz-Institut für Analytische Wissenschaften, Dortmund, Deutschland DOI 10.1055/s-0041-1723668

Einleitung NME-GPS ist ein multizentrisches, interdisziplinäres Forschungsprojekt, das darauf abzielt, zukünftig das diagnostische Prozedere bei PatientInnen mit neuromuskulären Erkrankungen zu verbessern.

Material/Methode Muskelbiopsien von Patienten mit neuromuskulären Erkrankungen werden mittels Proteom-Analyse (gezielter und ungezielter Ansatz) und durch Nutzung neuer mikroskopischer Verfahren wie CARS-Mikroskopie und Raman-Spektroskopie untersucht. Die gewonnenen biochemischen und bildgebenden Daten werden mit den Ergebnissen einer Exom-Analyse und klinischen Untersuchungsbefunden korreliert.

Ergebnisse Diese Korrelationsstudien dienen dazu, zukünftig mittels o.g. alternativer Messmethoden unter Verwendung einer minimalsten Menge an Muskelgewebe möglichst alle bekannten Proteine, die mit einer Ausprägung einer erblichbedingten Muskelerkrankung in Verbindung gebracht wurden, zu quantifizieren und zukünftig basierend auf dem Proteinmuster den Gendefekt vorhersagen zu können und umgekehrt (Implementierung von Vorhersagealgorithmen).

Diskussion Dadurch sollen den PatientInnen bzw. Familien ein breites und optimiertes diagnostisches Spektrum angeboten werden können. Die angewandte Methodik beinhaltet auch die Identifikation neuer krankheitsrelevanter Gene. Zudem sollen die Resultate der kombinierten Analysen dazu dienen, einheitliche Krankheitsmechanismen zu identifizieren, die zukünftig als Ansatzpunkte für therapeutische Konzepte dienen könnten.

\section{Differentialdiagnostik neuromuskulärer Erkrankungen}

\section{49 Risiko- und verlaufsmodulierende Faktoren der ALS - was wissen wir wirklich?}

Autoren Petri $S^{1}$

Institute 1 Medizinische Hochschule Hannover, Klinik für Neurologie,

Hannover, Deutschland

DOI 10.1055/s-0041-1723669

Amyotrophe Lateralsklerose (ALS) ist eine neurodegenerative Erkrankung mit kombinierter Affektion des ersten und zweiten Motoneurons, die sich mit rasch progredienten Paresen der gesamten Skelettmuskulatur manifestiert und meist durch eine Mitbeteiligung der Atemmuskulatur innerhalb weniger Jahren nach Symptombeginn zum Tod führt. Die Pathogenese der ALS ist bis heute nicht vollständig geklärt. Vermutet wird eine multifaktorielle Ätiologie, wobei bisher unter anderem genetische Faktoren, oxidativer Stress und ein veränderter RNA-Metabolismus als mögliche Einflussfaktoren diskutiert werden.

Verschiedene Studien haben gezeigt, dass bestimmte Komorbiditäten, insbesondere kardiovaskuläre Erkrankungen und Risikofaktoren wie die
Hypercholesterinämie bei ALS-Patienten im Vergleich zur Normalbevölkerung in veränderter Häufigkeit vorliegen und möglicherweise auch Einfluss auf den Krankheitsverlauf haben. Auch für Lebensstilfaktoren wie das Ausmaß körperlicher Aktivität, Ernährungsgewohnheiten, Nikotinkonsum, Lebensumfeld und Beruf konnte eine veränderte Häufigkeit bei ALS-Patienten im Vergleich zur Normalbevölkerung und eine Modifikation des Fortschreitens der Erkrankung nachgewiesen werden. Im Vortrag werden eigene Ergebnisse zu Risiko- und verlaufsmodulierenden Faktoren aus einer Patientenkohorte aus drei europäischen Ländern sowie weitere Daten aus der Literatur vorgestellt und diskutiert.

\section{51 Whole Genome Sequencing (WGS) - A Solved Case Of Dystrophinopathy In A Young Girl}

Authors Pluta $\mathrm{N}^{1}$, Zaum $A K^{1}$, Nanda $I^{1}$, Zimmer $\mathrm{F}^{1}$, von Moers $\mathrm{A}^{2}$, Rost $\mathrm{S}^{1}$

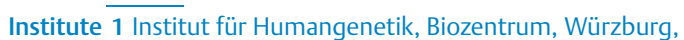

Deutschland; 2 DRK Kliniken Berlin Westend, Berlin, Deutschland

DOI 10.1055/s-0041-1723670

Introduction Whole Genome Sequencing (WGS) will be the method of choice in near future genetic diagnostics. Major challenges in WGS are interpreting and handling the large amount of data and finding the causative variant(s) in the haystack of variants. Here we present a case that was solved with WGS in which all other methods used did not yield definite results.

Material/Methods The patient is a 6 year old girl with muscle weakness and very high levels of serum creatine kinase $(C K)>20,000 U /$. Due to her symptoms and an almost completely skewed $\mathrm{X}$-inactivation a dystrophinopathy was strongly suggested. WGS was performed and analyzed with our software for routine NGS diagnostics.

Results We found a position in intron 54 of the DMD gene where a small intergenic sequence of chromosome 5 was detected. By performing Sanger sequencing we confirmed a reciprocal translocation involving th short arm of chromosome 5 and $\mathrm{X}$ with definite breakpoints. Previously performed cytogenetic analysis could not depict the evidence of a translocation between $5 p$ and Xp. An extended FISH analysis is essential to validate the translocation and will be useful to demonstrate the participation of the active chromosome $X$ in the translocation.

Discussion This case shows how important WGS will be or already is for genetic routine diagnostics. By performing WGS as a first step this case could have been solved faster and with higher accuracy than the stepwise application of conventional methods.

Neue therapeutische Prinzipien

\section{54 Realisierung einer Muskelstammzelltherapie}

Autoren Schöwel-Wolf $V^{1}$, Kieshauer ${ }^{1}$, Marg $A^{1}$, Bekele $B^{1}$, Ebert AK ${ }^{2}$, Spuler $\mathrm{S}^{1}$

Institutes 1 Experimental and Clinical Research Center, Charité

Universitätsmedizin Berlin und Max-Delbrück-Centrum für Molekulare Medizin,

Muscle Research Unit und Hochschulambulanz für Muskelkrankheiten, Berlin,

Deutschland; 2 Universitätsklinikum Ulm, Abteilung für Urologie und

Kinderurologie, Ulm, Deutschland

DOI 10.1055/s-0041-1723671

Einleitung Muskelschwund ist nicht therapierbar. Der Skelettmuskel besitzt eigenen Stammzellen, die Satellitenzellen. Bei Gesunden sind Satellitenzellen für die hohe Regenerationsfähigkeit des Gewebes verantwortlich. Therapeutisch waren diese Zellen bisher nicht nutzbar.

Methode Wir haben eine Technologie zur Herstellung hochregenerativer Satellitenzellen entwickelt. Unser Ziel ist die Entwicklung von Stammzelltherapien zur Behandlung von Muskelkrankheiten. Wir arbeiten an der Zulassung klinischer Studien.

Ergebnis Wir haben die einzelnen Herstellungsschritte für die nicht genetisch modifizierten Zellen an Arzneimittelstandards angepasst. Der genetische 
Modifikationsschritt wird derzeit adaptiert. Die unmodifizierten Zellen haben die präklinische Wirksamkeits- und Sicherheitsprüfung durchlaufen. Klinisch werden sie erstmals bei einer angeborenen Entwicklungsstörung zur Rekonstruktion eines kleinen Sphinktermuskels angewendet. Das regulatorische Beantragungsverfahren wird vorbereitet. Die Studieninfrastruktur ist aufgebaut. Die Phase I/Ila-Studie beginnt im Winter 21/22, finanziert durch das BMBF.

Schlussfolgerung In den 90ern ist die Entwicklung einer Muskelstammzelltherapie gescheitert. Seitdem wurde detailliert an diesem Zelltyp geforscht. Wir können jetzt reine, hochregenerative Satellitenzellen herstellen und diese mittels Gene Editing korrigieren. Die extrakorporale Geneditierung minimiert das Arzneimittelrisiko. Eine schnelle klinische Anwendung ist anzustreben.

\section{Platform Trials und Real-Life-Outcome}

\section{58 Behandlungsziele und Therapiezufriedenheit von adulten 5q-SMA-Patienten mit Nusinersen- Behandlung - Ergebnisse einer multi-zentrischen Kohortenstudie}

Autoren Meyer $\mathrm{T}^{1,2}$, Spittel $\mathrm{S}^{1,2}$, Uzelac $Z^{3}$, Hagenacker $\mathrm{T}^{4}$, Günther $\mathrm{R}^{5,6}$, Schreiber-Katz $\mathrm{O}^{7}$, Weiler $\mathrm{M}^{8}$, Steinbach $\mathrm{R}^{9}$, Weyen $\mathrm{U}^{10}$, Koch $J \mathrm{C}^{11}$, Kettemann $D^{1}$, Norden $\rfloor^{1}$, Dorst $\rfloor^{3}$, Ludolph $A C^{3}$, Stolte $B^{4}$, Osmanovic $A^{7}$, Petri $S^{7}$, Rödiger $A^{9}$, Grosskreutz $\rfloor^{9}$, Griep $R^{2}$, Gaudlitz $M^{2}$, Walter $B^{1}$, Münch $C^{1,2}$, Meier $A^{1}$

Institutes 1 Charité - Universitätsmedizin Berlin, Klinik für Neurologie, Ambulanz für ALS, SMA und andere Motoneuronerkrankungen, Berlin, Deutschland; 2 Ambulanzpartner Soziotechnologie APST GmbH, Berlin, Deutschland; 3 Universitätsklinikum Ulm, Klinik für Neurologie, Ulm, Deutschland; 4 Universitätklinikum Essen, Klinik für Neurologie, Essen, Deutschland; 5 Universitätsklinikum Carl Gustav Carus, Klinik für Neurologie, Dresden, Deutschland; 6 Deutsches Zentrum für Neurodegenerative Erkrankungen, Standort Dresden, Dresden, Deutschland; 7 Medizinische Hochschule Hannover, Klinik für Neurologie, Hannover,

Deutschland; 8 Universitätsklinikum Heidelberg, Klinik für Neurologie, Heidelberg, Deutschland; 9 Universitätsklinikum Jena, Klinik für Neurologie, Neuromuskuläre und Motoneuron Ambulanz, Jena,

Deutschland; 10 Berufsgenossenschaftliches Universitätsklinikum Bergmannsheil, Klinik für Neurologie, Ambulanz für ALS und andere Motoneuronerkrankungen, Bochum, Deutschland; 11 Universitätsmedizin Göttingen, Klinik für Neurologie, Göttingen, Deutschland

DOI 10.1055/s-0041-1723672

Hintergrund Bei Patienten mit adulter 5q-SMA und Nusinersen-Behandlung, sollten klinische Charakteristika, Behandlungsziele sowie Therapieerfahrungen systematisch erfasst werden.

Methode In eine prospektive Beobachtungsstudie wurden folgende Patienten eingeschlossen: 5q-SMA, Nusinersen-Behandlung, Teilnahme bei "Ambulanzpartner". Die Behandlungsziele und die Symptomschwere wurden durch den MYMOP, die Therapiezufriedenheit durch den TSQM-9 und die Weiterempfehlungswahrscheinlichkeit durch den NPS erfasst.

Ergebnisse 151 adulte 5q-SMA-Patienten wurden analysiert. Patienten mit SMA-Typ 3 (60\%) überwogen. Folgende Behandlungsziele wurden ermittelt: Kopfkontrolle $4 \%$, Bulbärfunktion 5\%, Armfunktion $22 \%$, Atemfunktion $5 \%$, Rumpffunktion $12 \%$, Beinfunktion $26 \%$, Allgemeinsymptome $26 \%$. Die mittlere Symptomschwere verbesserte sich im Therapieverlauf von 3,7 auf 3,3 MYMOP-Punkte $(p<0,001)$. Die Weiterempfehlungsrate von Nusinersen war sehr hoch: NPS +51. Im TSQM-9 zeigte sich eine hohe Zufriedenheit mit der Effektivität von Nusinersen $(66,5)$, die Anwenderfreundlichkeit wurde geringer bewertet $(49,7)$. Die Gesamtzufriedenheit war wiederum hoch $(74,3)$.

Diskussion Die Behandlungsziele von Nusinersen waren individuell und abhängig vom Typ und Schwergrad der SMA. Die Bewertung der
Anwenderfreundlichkeit war geringer und im Kontext der intrathekalen Applikation zu diskutieren. Dennoch waren die Gesamtzufriedenheit und die Weiterempfehlung der Nusinersen-Therapie bemerkenswert hoch.

\section{60 Real-world-Erfahrungen mit Onasemnogen Abeparvovec (Zolgensma ${ }^{\circledR}$ ) bei Spinaler Muskelatrophie (SMA)}

Autoren Weiß $C^{1}$, Becker $L^{1}$, Stoltenburg $C^{1}$, van der Stam $L^{1}$, Kaindl $A$ Institute 1 Charité - Universitätsmedizin Berlin, Neuropädiatrie, Berlin, Deutschland DOI 10.1055/s-0041-1723673

Fragestellung Die europäische Zulassung der Genersatztherapie mit Zolgensma ${ }^{\circledR}$ erfolgte für ein breiteres Patientenkollektiv als das in Studien untersuchte. Standardisierte Anwendungsbeobachtungen von Patienten $\geq 8$ Monaten bzw. $\geq 8,5 \mathrm{~kg}$ und nach Vorbehandlung mit Nusinersen sind somit essentiell.

Methoden Wir präsentieren motorische Funktionswerte (CHOP INTEND und motorische Meilensteine), Daten zu Ernährung und Atmung sowie klinische und paraklinische Nebenwirkungen der Patienten, die an der Charité Universitätsmedizin Berlin mit Zolgensma ${ }^{\circledR}$ behandelt und über mind. 60 Tage nachbeobachtet wurden (mind. $\mathrm{n}=16$ ).

Ergebnisse Alle bisher behandelten Patienten $(n=12)$ waren mit Nusinersen vorbehandelt. 11/12 zeigten einen signifikanten Anstieg des CHOP INTEND und 9/12 erreichten neue motorische Meilensteine. Bei allen Patienten mit vorbestehender Beatmungspflichtigkeit $(n=4)$ konnten Beatmungsparameter reduziert bzw. die Beatmungsdauer verkürzt werden. Bei 4/8 Patienten mit vorbestehender Dysphagie konnten eine Verbesserung des Schluckens beobachtet werden. Alle Patienten erlitten Nebenwirkungen, schwere Nebenwirkungen traten nicht auf.

Schlussfolgerungen Kinder, die unter einer Nusinersentherapie wenige oder keine Verbesserungen erzielen, können von Zolgensma® zusätzlich profitieren. Die Behandlung wurde von Patienten bis zu 37 Monaten und 14 kg gut toleriert. Engmaschige Nachkontrollen in einem spezialisierten neuromuskulären Zentrum sind zwingend erforderlich.

Hilft die Genetik bei der Abschätzung der Prognose?

\section{63 Clinicial, histological and molecular analysis of ANO5-myopathy}

Authors Richert $\mathrm{C}^{1}$, Schneider $\mathrm{I}^{2,3}$, Stoltenburg-Didinger $\mathrm{G}^{3}$, Klein $\mathrm{O}^{4}$, Zierz $S^{3}$

Institute 1 Krankenhaus Martha-Maria Halle-Dölau, Klinik für Kardiologie, Halle a. d. S., Deutschland; 2 Klinikum St. Georg Leipzig, Klinik für Neurologie, Leipzig, Deutschland; 3 Martin-Luther-Universität Halle- Wittenberg und Universitätsklinikum Halle, Universitätsklinik und Poliklinik für Neurologie, Halle a. d. S., Deutschland; 4 Charité - Universitätsmedizin Berlin, Cardioproteomics Unit, Research Field Cardiovascular System BCRT, Berlin, Deutschland DOI 10.1055/s-0041-1723674

Introduction Anoctamin 5 (ANO5) mutations cause muscular disorders that present with different phentotypes: Isolated hyper-CK-emia, proximal dystrophy of limb girdle muscles (LGMD) and distal muscle dystrophy of Mioshy type can occur. Anoctamin is a member of the ANO/TMEM16 family of anion channels. However, the mechanisms of ANO5-induced muscular damage remain elusive.

Methods 12 patients with genetically confirmed autosomal recessive mutations of ANO5 were clinically and histologically characterized. Proteomic composition of muscle tissue from 12 patients and 3 controls (w/o ANO5 mutation) were explored using liquid chromatography based mass spectroscopy (label free quantification). 
Results Patients presented with LGMD $(n=7)$, distal Mioshy type $(n=1)$, generalized weakness $(n=1)$, isolated hyper-CK-emia $(n=2)$. Most patients had amyloid deposits $(n=10)$. Mitochondrial abnormalities were present $(n=6)$ with COX-deficient fibers and mitochondrial accumulation. Mass spectroscopy revealed significantly diminished matrix protein levels of biglycan, tenascin-X and decorin compared to controls. Amyloid positive muscle tissue had higher levels of 14-3-3 and proteins of contractile apparat (actin, myosin, tropomyosin) than amyloid negative probes.

Conclusion ANO5 patients had variable clinical and histological phenotypes. Mitochondrial pathology presents as frequent finding. The results of mass spectroscopy analysis reflect muscular remodeling at protein level in ANO5-myopathy.

Video-Forum: gelöste und ungelöste Fälle

\section{69 Late-onset SEPN1-assozierte Myopathie infolge einer neuen compound heterozygoten Mutation im Selenon-Gen}

Autoren Saak $A^{1}$, Schaefer J ${ }^{1}$

Institute 1 Uniklinikum Dresden, Neurologie, Dresden, Deutschland DOI 10.1055/s-0041-1723675

Einleitung SEPN1-assoziierte Myopathien sind seltene kongenitale Myopathien mit Manifestation im Kindes- und Jugendalter. Beschriebene Phänotypen sind: (a) Rigid-spine Myopathie; (b) Multi-minicore Myopathie; (c) Mallory body-like Myopathie; (d) kongenitale Fasertypendysproportionierung. Alle publizierten adulten Fälle zeigten klinische Symptome bereits vor dem 15.Lebensjahr, Überleben bis zur 6.Dekade ist selten

Methodik/Kasuistik Ein 60-jähriger Patient entwickelte seit dem 50.LJ eine langsam progrediente axiale und Gliedergürtelschwäche mit Rigid-spine Syndrom. Bei noch erhaltener Gehfähigkeit manifestierte sich eine respiratorische Insuffizienz. Es erfolgte laborchemische, bioptische, bildgebende und molekulargenetische Aufarbeitung

Ergebnisse Laborchemisch fanden sich Blutbildauffälligkeiten: Makrozytose der Erythrozyten, Anisozytose der Thrombozyten, Lymphopenie. Molekulargenetisch wurden nicht vorbeschriebene compound heterozygote missenseMutationen (p.Leu331Pro; p.Ala440Thr) im Selenon-Gen mit Segregation in der Familie festgestellt. Diese wurden trotz unspezifischer Muskelbiopsie mittels Muskel-MRT als Biomarker als pathogen eingestuft

Diskussion Das primäre Manifestationsalter unseres Patienten ist mit 50 Jahren das bisher höchste beschriebene und erweitert so das phänotypische Spektrum des SEPN1-Mangels. Es fanden sich persistierende Auffälligkeiten des Blutbilds, wie sie bei Selen-Mangel bekannt sind, Selen-Substitution führte jedoch zu keiner Verbesserung

\section{Poster}

\section{Gewebspathologie}

\section{P 1 Post mortem histopathologische Analyse bei der Einschlusskörpermyositis}

Authors Kummer $K^{1}$, Glaubitz $S^{1}$, Götze R ${ }^{1}$, Zechel $S^{1}$, Schmidt ] ${ }^{1}$

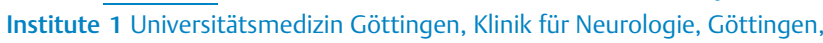
Deutschland

DOI 10.1055/s-0041-1723676

Einleitung Die Einschlusskörpermyositis ist eine inflammatorische Myopathie, welche zu einer langsam progredienten Tetraparese führt. Pathophysiologisch kommt es entzündlichen Infiltraten und degenerativen Muskelveränderungen. Pathologische Zellstressmechanismen scheinen eine wichtige Rolle in der Pathogenese einzunehmen. Die Abfolge der histopathologisch nachweisbaren Veränderungen ist weitestgehend ungeklärt.

Methoden Verschiedene Muskeln von 2 Patientinnen mit Einschlusskörpermyositis wurden post mortem histopathologisch auf entzündliche, degenerative und Zellstressmarker untersucht. Es erfolgte eine Korrelationsanalyse zwischen den Markern und in Bezug auf die klinisch zuvor gemessene Betroffenheit des Muskels.

Ergebnis Die histopathologischen Veränderungen variieren deutlich in den einzelnen Muskelgruppen. So zeigen einige Muskeln z.B. eine deutlich vermehrte Ablagerung von p62, während dies in anderen Muskeln nicht nachweisbar war. Zellstressmarker wie aB-Crystallin zeigen eine hohe Co-Expression zu Degenerations- und Inflammationsmarkern. In klinisch betroffenen Muskeln zeigen sich viele atrophe Fasern.

Diskussion Die Korrelationsanalyse zwischen klinischen Parametern und histopathologischen Daten wie Atrophie, Hypertrophie, p62, CD8+-Zellen, $\alpha B$-Crystallin, rimmed vacuoles und MHC-Klasse I in posthum entnommenen Muskeln von IBM-Patienten liefert einen wertvollen Beitrag zur Klärung der Pathogenese der Einschlusskörpermyositis.

\section{P 2 A specific type of capillary pathology is pathognomonic of scleroderma-associated myositis}

Authors Siegert $\mathrm{E}^{1}$, Uruha $\mathrm{A}^{2}$, Goebel $\mathrm{HH}^{2}$, Preuße $\mathrm{C}^{2}$, Casteleyn $\mathrm{V}^{1}$, Burmester $G R^{1}$, Schneider $U^{1}$, Dittmayer $C^{2}$, Stenzel $W^{2}$

Institute 1 Charité - Universitätsmedizin, Rheumatologie, Berlin,

Deutschland; 2 Charité - Universitätsmedizin, Neuropathologie, Berlin, Deutschland

DOI 10.1055/s-0041-1723677

Systemic sclerosis is a devastating chronic connective tissue disease featuring fibrosis, vasculopathy and autoimmunity. Affected patients show signs and symptoms of skin, multiple internal organs, and skeletal muscles. The vasculopathy is considered being obliterative, but its pathogenic underpinnings are still poorly understood. This may partially be due to limitations of conventional transmission electron microscopy. We ask wether in depth analysis of immune features and vascular ultrapathology can identfy a disease-specific pattern in Scleromyositis.

We apply conventional staining and immune labelling of skeletal muscle biopsies from patients with systemic sclerosis and muscle symptoms. We also use large-sclae Electron microscopy for evaluation of entire semithin sections and study $>1.000$ capillaries of patients and control biopsies.

In this presentation, we highlight skeletal muscle biopsies of Scleroderma patients presenting mild inflammatory features but prominent capillary alterations, which are at variance with pipestems. Large-scale electron microscopy and pan-and-zoom analysis, revealed a characteristic capillary morphology consisting of basement membrane thickening and reduplications, endothelial activation and pericyte proliferation.

We clearly show unique capillary pathology in skeletal muscles, which is specific for myositis in scleroderma, and discuss possible pathomechanisms.

\section{P 3 Effekte des selektiven iNOS-Inhibitors 1400W auf den Entzündungsstress im LPS-induzierten Myositismodell}

Autoren Pohlner J ${ }^{1}$, Schmidt J ${ }^{1}$, Zschüntzsch J

Institute 1 Universitätsmedizin Göttingen, Neurologie, Göttingen,

Deutschland

DOI 10.1055/s-0041-1723678

Einleitung In der Pathogenese der Einschlusskörpermyositis (IBM) wurde die vermehrte Produktion von Stickstoffmonoxid (NO) als Mediator zwischen degenerativen und entzündlichen Prozessen im Skelettmuskel 
identifiziert. Das Ziel der Arbeit war, die Effekte der iNOS-Inhibition mit N3-aminomethyl-benzyl-acetamidine (1400W) auf die Muskelpathologie in vivo zu untersuchen.

Methoden Bei doppel-transgenen MCK-APP/PS1-Mäusen wurde eine zusätzliche

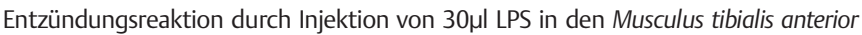
(TA) induziert. Eine s.c. Therapie mit 10 bzw. 30mg/kgKG $1400 \mathrm{~W}$ oder PBS als Kontrollsubstanz erfolgte 2x/d für zwei Tage unter Bestimmung der Griffkraft (GK). Immunhistochemische Färbungen und rt-PCR erfolgten nach TA-Entnahme. Ergebnisse Nach LPS- im Vgl. zur kontralateralen PBS-Injektion fand sich eine signifikante Überexpression proinflammatorischer Zytokine, Makrophagen und T-Zellen sowie eine hoch signifikante Zunahme des nekrotischen Areals im Muskelquerschnitt. 1400W erbrachte höhere Werte für die GK und einen Trend zur Reduktion der Entzündungsprozesse $(10 \mathrm{mg} / \mathrm{kgKG}$ 1400W).

Diskussion Das kombinierte, genetische und induzierte murine IBM-Modell spiegelt die humane Pathologie der IBM-Patienten besser wider als das rein genetische Mausmodell. Die Experimente unterstreichen die wichtige Rolle des NO-Stresses in der IBM-Pathologie und verweisen auf einen interessanten therapeutischen Ansatz im Sinne einer differenziert eingesetzten NO-Modulation.

\section{P 4 Der Kaliumkanal KCNK2 als Regulator für inflammatorische Prozesse in idiopathischen inflammatorischen Myopathien}

Autoren Müntefering $T^{1,2}$, Cengiz $D^{1}$, Nelke $C^{1}$, Meuth $S G^{2}$, Ruck $T^{1,2}$ Institutes 1 University Clinic Münster, translationale Neurologie, Münster, Deutschland; 2 University Clinic Düsseldorf, Klinik für Neurologie, Düsseldorf, Deutschland

DOI 10.1055/s-0041-1723679

KCNK2 gehört zur Gruppe der Zwei-Poren-Kaliumkanäle. Kürzlich konnte für KCNK2 eine regulierende Rolle für die Immunzellmigration an der Bluthirnschranke gezeigt werden. Idiopathische inflammatorische Myopathien (IIM) sind ebenfalls durch eine Immunzellinfiltration gekennzeichnet, so dass eine pathogenetische Rolle von KCNK2 in IIMs möglich ist. In Übereinstimmung, konnten wir die Expression von KCNK2 in primären murinen und humanen Skelettmuskel- und mikrovaskulären Endothelzellen auf Genund Proteinebene nachweisen. Unter inflammatorischen Bedingungen zeigten murine und humane Primärzellen eine Herabregulation der KCNK2 Expression. Durch verschiedene in vitro und in vivo Versuche konnten wir zeigen, dass durch eine pharmakologische Inhibition des Kanals die proinflammatorische Antwort von Muskel- und Endothelzellen gesteigert wird. Eine Aktivierung des Kanals hingegen führte nach einer Inflammation zu einem schnelleren Rückgang zur physiologischen Situation. Zusammenfassend konnten wir zeigen, dass die Modulation des KCNK2 Kanals einen erheblichen regulatorischen Einfluss auf die inflammatorische Antwort von Muskel- und Endothelzellen und hierdurch auch auf die Immunzellinfiltration. Dieses Verständnis könnte für neue, dringend benötigte therapeutische Ansätze der IIMs genutzt werden.

\section{P 5 Analyse des interstiellen Fibrosegrades bei kindlichen Kardiomyopathien in Abhängigkeit der verschiedenen Kardiomyopathie-Subtypen}

Autoren Keuper $\mathrm{M}^{1}$, Sehring $]^{1}$, Krück $S^{2}$, Dettmeyer $\mathrm{R}^{3}$, Schmidt $\mathrm{G}^{1}$, Rupp $S^{2}$, Schänzer $A^{1}$

Institutes 1 Justus Liebig Universität, Institut für Neuropatholgie, Gießen,

Deutschland; 2 Justus Liebig Universität, Kinderherzzentrum, Gießen,

Deutschland; 3 Justus Liebig Universität, Institut für Rechtsmedizin, Gießen,

Deutschland

DOI $10.1055 / \mathrm{s}-0041-1723680$
Einleitung Während bei Kardiomyopathien(CM) im Erwachsenenalter die Fibrosierung zur kardialen Dysfunktion beiträgt, ist sie bei Kardiomyopathien im Kindes- und Jugendalter wenig bekannt. Das soll in dieser Studie näher untersucht werden.

Methoden Untersucht wurden 21 explantierte Herzen von Patienten im Alter von 9,47 Jahren (J) (0,75- 32,25J), $33 \%$ weiblich (W). Die Erkrankungsdauer lag bei 2,90 J (0,17-12,75 J). 38\% hatten eine dilatative CM (DCM), 19\% eine hypertrophe CM (HCM) und bei $43 \%$ war die kardiale Dysfunktion durch einen angeborenen Herzfehler (CHD) bedingt. Zusätzlich wurde postmortales Herzgewebe von 9 Probanden ohne Herzerkrankung analysiert (4,94 J (0,42-16 J) $22 \%$ W). An Masson-Trichrom gefärbten Schnitten wurde der interstitielle Fibrosegrad (FG) untersucht und mit klinischen Daten korreliert.

Ergebnisse Im Vergleich zur Kontrollgruppe (1\% +/- 0,55\%) konnten in allen 3 Gruppen signifikant erhöhte Fibroseanteile gezeigt werden: DCM $12 \%(+/-15 \% p=0.05)$, HCM $13 \%(+/-9 \% p=0.045)$, CHD $8 \%(+/-5 \%$ $\mathrm{p}=0.002)$. Alter und Erkrankungsdauer korrelierten dagegen nicht signifikant mit dem FG.

Diskussion Bei kindlichen Kardiomyopathien zeigt sich eine Fibrosierung, wobei in der untersuchen Kohorte kein signifikanter Einfluss des Alters oder der Erkrankungsdauer zu erkennen ist. Dies ist möglicherweise durch die große Heterogenität der Proben bedingt. Fibrosierungsprozesse im Herzmuskel sollten Gegenstand weiterer Forschung sein und an größeren Kohorten untersucht werden.

\section{P 6 Untersuchungen des Zytoskeletts von sensorischen Nervenfaserendigungen in Hautbiopsien von Gesunden}

Autoren Metzner $\mathrm{K}^{1}$, Rödiger $\mathrm{A}^{1}$, Gaur $\mathrm{N}^{1}$, Steinbach $\mathrm{R}^{1}$, Axer $\mathrm{H}^{1}$, Brill $\mathrm{MS}^{2}$, Grosskreutz J ${ }^{1}$

Institutes 1 Universitätsklinikum Jena, Jena, Deutschland; 2 Technische Universität, München, Deutschland

DOI 10.1055/s-0041-1723681

Einleitung Neurone mit langen Fortsätzen scheinen bezüglich der Primärmanifestation in neurodegenerativen Erkrankungen, wie zum Beispiel Amyotrophe Lateralsklerose oder Hereditäre Spastische Paraplegie, besonders vulnerabel zu sein. Ein etablierter Biomarker für neuroaxonale Schäden ist u.a. Neurofilament NfL, eine Komponente des Zytoskeletts (Bacioglu et al. 2016). In dieser Studie fokussieren wir uns auf die Expression von Neurofilamenten, Aktin, Mikrotubuli in sensorischen Nervenfaserendigungen gesunder Versuchspersonen, um die Alters- und Längenabhängigkeit zu erfassen.

Methoden Hautbiopsien (Ober- und Unterschenkel) von 27 Männern (Durchschnittsalter 54,2 \pm 18,9 a, von 24 a bis 79 a) wurden gefärbt ( $\beta$ IIITubulin, nicht-phosphoryliertes Neurofilament npNfH, Aktin), konfokale Bilder der Dermis aufgenommen und die Grauwerte und Kaliber quantitativ analysiert.

Ergebnisse Die Grauwerte der Immunfluoreszenzfärbung zeigen altersabhängig einen signifikanten Anstieg ( $\beta$ III-Tubulin: $p=0,001$; $n$ pNfH: $p=0,004$; Aktin: $\mathrm{p}<0,001)$. Das Axonkaliber korreliert ebenfalls positiv mit dem Alter ( $\mathrm{p}$ $<0,001)$.

Diskussion Unsere Ergebnisse deuten auf eine Verknöcherung des axonalen Zytoskeletts hin, die die Funktionalität des Zytoskeletts ebenso wie das Axonkaliber in höherem Alter beeinflusst. Dies könnte im Zusammenhang mit der steigenden Inzidenz neurodegenerativer Erkrankungen im Alter stehen. Wir planen, unsere bisherigen Ergebnisse in einer zweiten Kohorte zu konsolidieren. 


\section{P 7 Kapillarmikroskopische Untersuchung der mikrovaskulären Architektur bei nicht-rheumatischen neuromuskulären Erkrankungen Microvascular changes in patients with non-inflammatory neuromuscular diseases}

\author{
Autoren Penka $D^{1,2}$, Hasseli $R^{2}$, Kalms $N^{1}$, Hermann $W^{2}$, Schänzer $A^{3}$, \\ Müller-Ladner $\mathrm{U}^{2}$, Krämer $\mathrm{HH}^{1}$ \\ Institutes 1 Justus-Liebig-Universität, Abteilung für Neurologie, Gießen, \\ Deutschland; 2 Justus-Liebig-Universität, Abteilung für Rheumatologie und \\ klinische Immunologie, Campus Kerckhoff, Bad Nauheim, \\ Deutschland; 3 Justus-Liebig-Universität, Institut für Neuropathologie, Gießen, \\ Deutschland \\ DOI 10.1055/s-0041-1723682
}

Introduction Microvascular changes (MC) are frequently present in inflammatory rheumatic diseases (IRD, e.g. systemic sclerosis). Alterations of capillary architecture could also be detected in non-inflammatory neuromuscular diseases (NI-NMD) ${ }^{1}$. The aim of this study was to evaluate systematically MC in NI-NMD.

Material/Methods We already included 38 patients (39\% myasthenia gravis (MG)). Nailfold videocapillaroscopy (NVS) was performed to evaluate MC. A standardized questionnaire was used to assess history of Raynaud"s phenomenon, information about the patient's underlying disease, comorbidities, medication and smoking. In addition, laboratory parameters, autoantibodies, MRI and histological findings were assessed. Patients with polyneuropathies, diabetes, IRD were excluded. MC were evaluated by using a validated microangiopathy evolution score (MES) ${ }^{2}$.

Results In $95 \%$ (36/38) of the patients MC could be detected which included tortousity (81\%), ramification (100\%), irregular enlargement (75\%), bulging (92\%), elongation (78\%), bleedings (67\%), edema (75\%), but no giant capillary and only six bushy capillaries. The MES ranged between 1,3 und 4,3 ( $83 \%$ from 2,3 to 4$)$.

Discussion MC are present in NI-NMD including MG, and mimic the alterations described in IRD. Further investigations are ongoing to assess if specific changes are associated with specific type of NI-NMD.

[1] Paula et al., Rheumatology 2016;55:1911-1912.

[2] Sulli et al. Ann Rheum Dis 2008;67(6):885-7

Devices in Diagnose und Behandlung

\section{P 8 Somatosensory-related but not vestibular-related spatial navigation is impaired in patients with myasthenia gravis}

Authors Müller $\mathrm{P}^{1,2}$, van Wickeren $\mathrm{N}^{1,2}$, Klaus $\mathrm{B}^{1,2}$, Dordevic $\mathrm{M}^{1,2}$, Schmicker $\mathrm{M}^{1}$, Vielhaber $S^{1,2,3}$, Schreiber $S^{1,2,3}$, Müller $N^{1,2,3}$

Institute 1 Deutsches Zentrum für Neurodegenerative Erkrankungen, AG Neuroprotektion, Magdeburg, Deutschland; 2 Universitätsklinikum

Magdeburg, Klinik für Neurologie, Magdeburg, Deutschland; 3 Center for behavioral brain sciences (CBBS), Magdeburg, Deutschland

DOI 10.1055/s-0041-1723683

Introduction: Myasthenia gravis (MG) is an autoimmune disease affecting the postsynaptic membrane at the neuromuscular junction. The predominant clinical manifestation is muscle weakness, reinforced by muscle activity. Additionally, cognitive deficits, autonomic dysfunction and peripheral neuropathy have been described. Here, we investigate the somatosensory and vestibular function in a spatial navigation task in MG patients.

Materials \& Methods: Fifteen MG patients (age: $66.89 \pm 13.77,12$ male) were compared to age-, sex- and education-matched healthy controls (age: $66.73 \pm$ 14.25). All patients received cholinesterase inhibitors. The patients" clinical manifestation was assessed through electroneurography (ENG), muscle strength according to the medical research council and pallaesthesia.
Somatosensory and vestibular function was quantified applying the triangle completion task (Dordevic et al. 2017).

Results: There were no significant differences between controls and $M C$ patients regarding muscle strength, tendon reflexes and pallaesthesia. Additionally, peripheral neuropathy was excluded through ENG. In the triangle completion task MG patients displayed significant deteriorations in their somatosensory ( $p=.016 ; d=0.83$ ), but not in their vestibular functions.

Discussion: Our results demonstrate that MG is associated with somatosensory deteriorations. Future research is needed to replicate this finding in a larger and independent cohort and to investigate the underlying mechanisms.

\section{P 9 Implantation eines intrathekalen Kathetersystems zur Applikation von Nusinersen bei Kindern mit Spinaler Muskelatrophie Typ 2}

Autoren Husain RA ${ }^{1}$, Reichart $R^{2,3}$, Al Safatli $D^{3}$, Senft $C^{3}$, Mehlhorn $C^{1}$, de Vries $\overline{\mathrm{H}^{1} \text {, Brandl }} \mathrm{U}^{1}$

Institutes 1 Universitätsklinikum Jena, Klinik für Neuropädiatrie, Jena, Deutschland; 2 Rhön-Klinikum, Klinik für Neurochirurgie, Bad Neustadt a. d. Saale, Deutschland; 3 Universitätsklinikum Jena, Klinik für Neurochirurgie, Jena, Deutschland

DOI 10.1055/s-0041-1723684

In unserer Klinik für Neuropädiatrie wurden von Juli 2017 bis Oktober 2020 bei 21 Kindern mit Spinaler Muskelatrophie insgesamt 202 intrathekale Nusinersen-Gaben durchgeführt, Alter bei Behandlungsbeginn 5 Monate bis 16 Jahre. Dabei wurde 2 Patienten ein intrathekales Medikamenten-Portsystem implantiert.

Beide Patienten mit SMA Typ 2 und neuromyogene Skoliose stellten sich im Alter von 10 Jahren zur Einleitung einer Therapie mit Nusinersen vor. Im Alter von 7 bzw. 9 Jahren war extern eine Wirbelsäulenoperation erfolgt (dorsaler Stabilisierung BWK2-SWK1 mit einem Schrauben-Stab-System bzW. partielle dorsaler Korrektur mittels Implantation von magnetisch distrahierbaren "Growing-Rods" BWK3/4-LWK3/4).

Nach LWS-CT erfolgte die Implantation eines intrathekalen Katheters und Anschluss an einen abdominell gelegenen Port, hierüber Nusinersen-Therapie gemäß Empfehlung (bisher 7 bzw. 8 Gaben). Prophylaktische Portspülung im Intervall von 2 Monaten. Im Beobachtungszeitraum (1 Jahr 8 Monate bzw. 1 Jahr 3 Monate) bei Patient 1 einmalig starke Kopfschmerzen nach Portspülung, bei Patient 2 einmalig frustrane Portpunktion zur Spülung bei ängstlichschmerzempfindlicher Patientin, ansonsten unkomplizierter Verlauf.

Bei komplexen Wirbelsäulenverhältnissen senkt eine intrathekale Applikation von Nusinersen über ein implantiertes Portsystem die Strahlenbelastung gegenüber Durchleuchtungs- und CT-gestützten Lumbalpunktionen und ist insgesamt eine atraumatische und sichere Alternative.

\section{P 10 Ultrasound of peripheral nerves as preclinical marker for disease progression in mutant SOD1G93A mice?}

Autoren Thau-Habermann $\mathrm{N}^{1}$, Binz $\mathrm{C}^{1}$, Rückoldt $\mathrm{J}^{1}$, Friedrich $\mathrm{A}^{1}$, Gschwendtberger $\mathrm{T}^{1}$, Huang $Z^{2}$, Haastert-Talini $\mathrm{K}^{2}$, Brandes $\mathrm{G}^{2}$, Schreiber $\mathrm{S}^{3}$, Petri $S^{1}$

Institutes 1 Medizinische Hochschule Hannover, Neurologie, Hannover, Deutschland; 2 Medizinische Hochschule Hannover, Neuroanatomie und Zellbiologie, Hannover, Deutschland; 3 Otto von Guericke Universität, Neurologie, Magdeburg, Deutschland

DOI 10.1055/s-0041-1723685

Introduction In the last decade few studies have been published on sonographic evaluation of peripheral nerves in patients suffering from ALS. In the majority, the cross-sectional area (CSA) of a variety of different nerves was analysed in ALS patients compared to controls or throughout disease progression. Most studies showed CSA reduction in ALS patients. Schreiber and col., on the other 
hand, described an inflammatory subtype of ALS patients with increase in CSA, that also was detected in a large proportion of patients carrying SOD1 mutations. In relation to these findings we are currently investigating the sciatic nerve of ALS transgenic mice by ultrasound.

Methods Ultrasound data are set in the context of standard parameters in this animal model at different disease stages: Motor function tests, phenotypic data, nerve conduction studies and histological data from the sciatic nerve, spinal cord and gastrocnemius muscle. Diameter and CSA of sciatic nerve are evaluated and grey-scale analysis of CSA is performed.

Results Our preliminary results indicate that ultrasound measurements can be used as sensitive and non-invasive (pre-) clinical marker of disease progression. Discussion A possible effect of an experimental treatment could therefore be detected earlier and more sensitively. Moreover, our multi-modal analyses will provide further insight into the correlation of neurophysiological and morphological/histopathological data in the mutant SOD1 mouse model of ALS.

\section{P 11 Robotik bei der ALS - Nutzenerwartung an die zukünftige Versorgung mit einem Roboterarm}

Autoren Maier $\mathrm{A}^{1}$, Spittel $\mathrm{S}^{1,2}$, Gaudlitz $\mathrm{M}^{2}$, Weyen $\mathrm{U}^{3}$, Grosskreutz $\rfloor^{4}$, Grehl $\mathrm{T}^{5}$, Weydt $\mathrm{P}^{6}$, Petri $\mathrm{S}^{7}$, Schreiber-Katz $\mathrm{O}^{7}$, Baum $\mathrm{P}^{8}$, Metelmann $\mathrm{M}^{8}$, Kettemann $D^{1}$, Norden $J^{1}$, Walter $B^{1}$, AD Sperfeld ${ }^{1,9}$, Koc $Y^{1}$, Münch $C^{1,2}$, Meyer $\mathrm{T}^{1,2}$

Institutes 1 Charité - Universitätsmedizin Berlin, Klinik für Neurologie, Ambulanz für ALS, SMA und andere Motoneuronerkrankungen, Berlin, Deutschland; 2 Ambulanzpartner Soziotechnologie APST GmbH, Berlin, Deutschland; 3 Berufsgenossenschaftliches Universitätsklinikum Bergmannsheil, Klinik für Neurologie, Ambulanz für ALS und andere Motoneuronerkrankungen Bochum, Deutschland; 4 Universitätsklinikum Jena, Klinik für Neurologie, Neuromuskuläre und Motoneuron Ambulanz, Jena, Deutschland; 5 Alfried Krupp Krankenhaus, Klinik für Neurologie, Ambulanz für ALS und andere Motoneuronerkrankungen, Essen,

Deutschland; 6 Universitätsklinikum Bonn, Klinik für Neurodegenerative Erkrankungen, Bonn, Deutschland; 7 Medizinische Hochschule Hannover, Klinik für Neurologie, Hannover, Deutschland; 8 Universitätsklinikum Leipzig, Klinik und Poliklinik für Neurologie, ALS-Ambulanz, Leipzig,

Deutschland; 9 Sächsisches Krankenhaus Altscherbitz, Klinik für Neurologie,

Schkeuditz, Deutschland

DOI 10.1055/s-0041-1723686

Einleitung Mit dem Roboterarm steht ein defizitorientiertes Hilfsmittel zur Verfügung, das krankheitsbedingte Funktionsverluste teilweise kompensieren und ausgleichen kann. Die Beobachtungsstudie erfasst die Nutzenerwartung gegenüber der zukünftigen Versorgung mit einem Roboterarm bei ALS-Patienten. Methode Es wurde eine prospektive Kohortenstudie an 8 ALS-Zentren realisiert. Einschlusskriterien: ALS, Indikation für einen Roboterarm. Das Funktionsdefizit wurde durch die ALS-Skala (ALSFRS-EX, 0-60 Punkte) und die subjektive Nutzenerwartung wurde durch eine Nummerische Rating Skala (0-10 Punkte) erfasst.

Ergebnisse 58 Patienten wurden analysiert (ALSFRS-EX: 30, \pm 10 ). Mehr als 90 \% würden den Roboterarm für körpernahe Bewegungen (97\%), zum Hantieren von Getränken und Gegenständen, Öffnen von Türen sowie zum Drücken von Knöpfen (je 93\%) nutzen. Weitere Nutzenerwartungen sind Essen anreichen (84\%), Bewegen des eigenen Körpers (74\%), Beschäftigung (76\%) und Körperpflege (69\%). 95\% der Patienten bewerten die Versorgung mit einem Roboterarm als sehr wichtig.

Diskussion Die Möglichkeit der Versorgung mit einem Roboterarm wird von fast allen Patienten als sehr wichtig eingestuft. Getränke anreichen, körpernahe Bewegungen oder Gegenstände hantieren stellen die größte Nutzenerwartung dar.

\section{P 12 Erfassung von Heilmitteln und alternativen Therapien bei neuromuskulären Erkrankungen}

Autoren Böhne $L^{1}$, Baum $P^{1}$, Schröter $C^{2}$, Wirner $C^{3}$ Institutes 1 Universitätsklinikum Leipzig, Klinik und Poliklinik für Neurologie, Leipzig, Deutschland; 2 Klinik Hohe Meißner, Neurologie, Bad SoodenAllendorf, Deutschland; 3 Friedrich- Baur- Institut, München, Deutschland DOI 10.1055/s-0041-1723687

Fragestellung Erfassung und Vergleich der Anwendung und Zufriedenheit von Heilmitteln und alternativen Therapien

Methoden Fragebögen in digitaler (Website des DGM) und analoger Form Ergebnisse Bisher konnten 153 Fragebögen ausgewertet werden. Die häufigsten Diagnosen waren hierbei Muskeldystrophien (26\%), myotone Dystrophien (19\%) und Motoneuronerkrankungen (24\%). Mehr als die Hälfte der Patienten nutzten ausschließlich konventionelle Heilmittel (59\%), 36\% konventionelle Therapien in Kombination mit alternativen Therapien, nur $4 \%$ gaben eine ausschließliche Nutzung von alternativen Therapien an. Bei den Heilmitteln wurde die Physiotherapie mit $82 \%$ genutzt.

Dabei wurden am häufigsten und mit größter Zufriedenheit die Physiotherapie nach Bobath (56\%), am Gerät (45\%), und im Bewegungsbad (26\%) angegeben. Weitere Heilmittel wie Ergotherapie (26\%) und Logopädie (15\%) wurden weniger häufig genutzt, das Feedback fiel jedoch positiv aus. Bei den Alternativen Therapien wurden physikalische Therapien wie manuelle Therapien (51\%), Massagen (53\%) und progressive Muskelentspannung (32\%) am häufigsten genutzt. Trotz positiven Feedbacks für Alternativtherapien wurde die progressive Muskelentspannung am häufigsten kritisiert.

Schlussfolgerungen Über 80 \% der befragten Patienten nutzen Heilmittel. Am besten wurden die Physiotherapie am Gerät, nach Bobath und im Bewegungsbad bewertet. Alternative Therapien wurden besonders in Kombination mit konventionellen Therapien eingesetzt, wobei vor allem die physikalischen Therapien mit großer Zufriedenheit angewendet wurden.

\section{P 13 suMus, ein digitales Ökosystem rund um die Quantifizierung der Muskelaktivität}

Autoren Gazzerro $\mathrm{E}^{1}$, Herze $\mathrm{R}^{2}$, Maron $\mathrm{E}^{3}$, Spuler $\mathrm{S}^{1}$

Institutes 1 Muscle Research Unit, ECRC, Charite Universitätsmedizin und Max Delbrück Center für Molekulare Medizin, Berlin,

Deutschland; 2 Humanity\&Tech GmBH, Utting am Ammersee,

Deutschland; 3 Elan-Physio, Berlin, Deutschland

DOI 10.1055/s-0041-1723688

Die raschen Fortschritte von Gen- und Stammzelltherapien bei genetischen Muskelkrankheiten erfordern eine Aktualisierung der Qualität der klinischen Studien, mit denen die Wirksamkeit dieser neuen Behandlungen getestet werden soll. Präzisere, schnellere und personalisierte Messungen wie z.B. Beweise aus der realen Welt müssen eingeführt werden. Unser Zielprodukt, suMus, ist ein sensorgestütztes System für motorische Signale, das im häuslichen/sozialen Umfeld des Patienten eingesetzt wird. Die erste Phase des Projekts bestand darin, die Zusammenarbeit/Gemeinschaft von Patienten, Therapeuten und Ärzte zu stärken. Um dieses erste Ziel zu erreichen, stellten wir eine webbasierte Plattform her für Telephysiotherapie für Patienten mit neuromuskulären Krankheiten. Die Plattform, in die sich Therapeuten und ihre PatientInnen einloggen, bietet 64 Kurze Lehr-Videos mit Übungen zum Training des Arms, des Beins, der Atmung und der Rumpfmuskulatur, sodass Therapeuten einen maßgeschneiderten Übungsplan für Patienten designen können. suMus registriert die Frequenz der vorgenommenen Übungen und das damit verbundene Patientenfeedback und stellte die Daten den Therapeuten und dem neuromuskulären Zentrum zur Verfügung. suMus ergänzt die reguläre Physiotherapie. Die von suMus generierten Daten werden den Krankheitsverlauf mit Daten aus der realen Welt und Patientenberichten überwachen und die Patienten bei der Durchführung individuell angepasster täglicher körperlicher Aktivitäten unterstützen 


\section{P 14 Fulminanter Verlauf einer Chronisch-aktiven Ebstein-Barr-Virus-Infektion mit Myositis und Hämophagozytotischer Lymphohistiozytose}

Autoren Kleeberg $\mathrm{K}^{1}$, Kendzierski $\mathrm{T}^{1}$, Mensch $\mathrm{A}^{1}$, Nägel $S^{1}$, Emmer $A^{1}$, Stoltenburg-Didinger $\mathrm{G}^{2}$, Zierz $\mathrm{S}^{1}$

Institutes 1 Universitätsklinikum Halle (Saale), Klinik und Poliklinik für

Neurologie, Halle a. d. S., Deutschland; 2 Charité Universitätsmedizin Berlin,

Institut für Zell- und Neurobiologie, Berlin, Deutschland

DOI 10.1055/s-0041-1723689

Einleitung Bei der seltenen chronisch-aktiven Verlaufsform einer Ebstein-BarrVirus-Infektion (CAEBV) kann es neben den Symptomen einer infektiösen Mononukleose auch zu einer Beteiligung verschiedener Organe (u.a. des Muskels) kommen. Eine Komplikation der CAEBV mit hoher Mortalität ist die Hämophagozytotische Lymphohistiozytose (HLH).

Methode Fallvorstellung einer 64-jährigen Patientin mit initial Rötung und Schmerzen am rechten Oberschenkel sowie Fieber.

Ergebnisse Bei Aufnahme Körpertemperatur bis $38,3{ }^{\circ} \mathrm{C}$, Schwäche der Hüftbeugung rechts und deutlich erhöhte CK (17,32 $\mu \mathrm{kat} / \mathrm{l}$, normal < 2,85 $\mu \mathrm{kat} / \mathrm{l})$. Histologisch Bild einer Myositis mit endomysialer Infiltration CD8+ T-Zellen und CD68+ Monozyten/Makrophagen. Im Liquor bei zunehmender Vigilanzminderung Nachweis von EBV-DNA. Im Verlauf entsprechend der Diagnosekriterien Feststellung einer HLH. Die immunsuppressive Therapie mit Rituximab, Dexamethason, Etoposid, MTX intrathekal, Ciclosporin A und Pentaglobin, sowie Antiinfektiva (u.a. Ganciclovir, Foscarnet) ohne Effekt, die Patientin verstarb nach sechsmonatigem Krankheitsverlauf.

Diskussion Trotz aktuell noch ausstehendem Nachweis der EBV-DNA im Muskel kann die histologisch nachgewiesene Myositis sehr wahrscheinlich im Zusammenhang mit der CAEBV gesehen werden. Dieser Fall unterstreicht die Bedeutung der EBV-Myositis als Differenzialdiagnose bei gleichzeitigem Vorliegen einer Myositis und von Allgemeinsymptomen (Fieber, Vigilanzminderung).

Surrogatmarker

\section{P 15 Hochauflösende Nerven- und Muskelsonographie als Marker für Differentialdiagnostik und Progression bei hereditären Polyneuropathien}

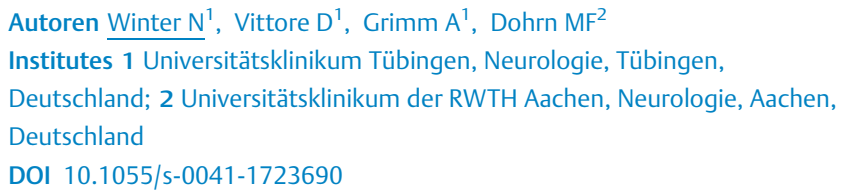

and CMTES $(p<0.0001)$. Am häufigsten war der Tibialis anterior Muskel verändert.

Diskussion Der Nerven- und Muskelultraschall ist ein geeignetes diagnostisches Mittel in der Unterscheidung und Verlaufsprogression genetisch bedingter und erworbener Polyneuropathien.

\section{P 16 Untersuchung mikrostruktureller Veränderungen ALS-spezifischer Traktsysteme bei asymptomatischen ALS-Mutationsträgern}

\section{Autoren Kassubek J $]^{1}$, Ludolph $\mathrm{AC}^{1}$, Müller $\mathrm{HP}^{1}$}

Institute 1 Universität Ulm, Neurologie, Ulm, Deutschland

DOI 10.1055/s-0041-1723691

Einleitung Neuropathologische Studien bei der ALS zeigen, dass die ALS in eine regionenspezifische Sequenz von vier Stages nach Braak et al. eingeteilt werden kann. Der in vivo-Transfer dieses Propagationsschemas ist mittels DTIbasierter Analyse spezifischen Traktsystemen erfolgt [1]. Diese Studie untersucht, ob bereits bei asymptomatischen ALS Mutationsträgern Veränderungen in den Traktsystemen detektiert werden können.

Methoden Die Analyse der fraktionellen Anisotropie (FA) wurde mittels eines ganzhirnbasierten voxelweisen Vergleichs (WBSS) sowie einer Tract-of-interest (TOI)-Analyse für 45 positiv getestete asymptomatische ALS Mutationsträger gegen Kontrollpersonen durchgeführt, um spezifische Veränderungen der Weißen Substanz darzustellen. Zusätzlich konnte eine longitudinale Analyse mit einem Zeitintervall von 300 Tagen von Daten von 20 positiv getesteten asymptomatischen ALS Mutationsträgern durchgeführt werden.

Ergebnisse Weder durch WBSS noch durch die TOI-basierte Analyse konnten signifikante charakteristische Veränderungsmuster für asymptomatische ALS Mutationsträger festgestellt werden.

Diskussion Diese Studie zeigt, dass mikrostrukturelle Veränderungen in den ALS-spezifischen Traktsystemen bei asymptomatischen Mutationsträgern nicht DTI-basiert fassbar sind, so dass diese Traktveränderungen erst mit Beginn des symptomatischen Krankheitsstadiums detektierbar zu sein scheinen. [1] Kassubek J, et al. J Neurol Neurosurg Psychiatry 2018; 89:374-381.

\section{P 17 Zusatznutzen quantitativer MRT-Analyseverfahren bei seltenen Muskelerkrankungen am Beispiel der MATR3-assoziierten distalen Myopathie}

Autoren Koester $\mathrm{F}^{1,2}$, Naegel $\mathrm{S}^{1}$, Kraya $\mathrm{T}^{1,3}$, Müller $\mathrm{T}^{1,4}$, Zierz $\mathrm{S}^{1}$, Stoevesandt $\mathrm{D}^{2}$, Mensch $\mathrm{A}^{1}$

Institutes 1 Universitätsklinikum Halle (Saale), Universitätsklinik und Poliklinik für Neurologie, Halle a. d. S., Deutschland; 2 Universitätsklinikum Halle (Saale), Universitätsklinik und Poliklinik für Radiologie, Halle a. d. S.,

Deutschland; 3 Krankenhaus St. Georg, Klinik für Neurologie, Leipzig,

Deutschland; 4 Ruppiner Kliniken, Klinik für Neurologie, Neuruppin, Deutschland

DOI 10.1055/s-0041-1723692

Fragestellung Der MRT kommt ein zunehmender Stellenwert in der Diagnostik und Verlaufsbeurteilung muskulärer Erkrankungen zu. Rezente Studien an großen Kohorten häufiger Myopathien zeigen einen Zusatznutzen quantitativer Analyseverfahren. Der Nutzen dieser Verfahren für seltene Myopathien ist nicht etabliert. Ziel der Studie war daher die Evaluation quantitativer Analyseverfahren für die sehr seltene MATR3-Myopathie in Hinblick auf Diagnosesicherung und Krankheitsschwere.

Methoden 15 Patienten mit MATR3-Myopathie (25-79 Jahre, 7 weiblich) wurden mit Ganzkörper-MRT untersucht. Der muskuläre Fettanteils wurde mittels Dixon-Methode in 7 ausgewählten Muskeln quantifiziert, als Kontrollgruppe dienten 60 gesunde Probanden.

Ergebnisse Die Analyse des muskulären Fettanteils erlaubte eine Unterscheidung zwischen krankheits- und altersbedingten Veränderungen. Entsprechend der klinischen Manifestation zeigten sich deutliche Veränderungen nach dem 35. Lebensjahr. Weiterhin ergaben sich messbare Veränderungen 
in ausgewählten Muskeln bereits bei Patienten unter 35 Jahren. Mit nur 4 Indikatormuskeln (Mm. gluteus minimus, semimembranosus, gastrocnemius und longissimus thoracis) war eine Diagnosestellung mit hoher Spezifität und Sensitivität möglich.

Schlussfolgerung Die Studie bestätigt den Stellenwert der Ganzkörper-MRT und der Quantifizierung des Muskelfettanteils zur frühen diagnostischen Sicherung und zur Verlaufsbeurteilung bei der seltenen MATR3-assoziierten distalen Myopathie.

\section{P 18 Signaturen der Krankheitsaggressivität von Amyotropher Lateralsklerose in der weißen Substanz: eine Anwendung des D50 Krankheitsprogressionsmodells}

Autoren Steinbach $\mathrm{R}^{1}$, Gaur $\mathrm{N}^{1}$, Roediger $\mathrm{A}^{1}$, Mayer $\mathrm{TE}^{2}$, Witte $O \mathrm{~W}^{1}$, Prell $T^{1}$, Grosskreutz ]

Institutes 1 Universitätsklinikum Jena, Hans-Berger-Klinik für Neurologie, Jena, Deutschland; 2 Universitätsklinikum Jena, Neuroradiologie, Jena, Deutschland DOI 10.1055/s-0041-1723693

Einleitung Bisherige Magnetresonanztomografie (MRT)-Studien zu Amyotropher Lateralsklerose (ALS) haben Verbindungen zwischen Strukturveränderung und Klinik hergestellt, allerdings ließ der interindividuell hoch-variable Erkrankungsverlauf der ALS robuste Korrelationen bislang nicht zu. Daher wurde hier das D50 Modell angewendet, das einerseits distinkte Parameter der Krankheitsaggressivität andererseits Quantifizierungen der Krankheitsakkumulation erlaubt. Methoden Diffusions-Tensor-Imaging (DTI) Daten von 145 Patienten und 69 Kontrollen wurden mittels Tract-Based-Spatial-Statistics analysiert. Kontraste wurden zwischen Patienten und Kontrollen sowie in der ALS-Kohorte basierend auf der individuellen Krankheitsakkumulation (Krankheits-Phase I und II) und der Krankheitsaggressivität (D50-Wert) berechnet.

Ergebnisse ALS-Kontroll-Vergleiche zeigten eine weitreichende ALS-Pathologie innerhalb der weißen Substanz auf. Innerhalb dieser Fasertrakte konnten auch Korrelation mit der Krankheitsakkumulation aufgezeigt werden, bedingt durch Patienten in Phase I. Es gab hingegen keine signifikanten DTI-Veränderungen im Vergleich von Phase II mit Phase I Patienten. Höhere Krankheitsaggressivität (D50 < 30 Monate) war durch robuste DTIVeränderungen in bifrontoparietalen Fasertrakten reflektiert, die in Regressionsanalysen bestätigt wurden.

Diskussion Das D50 Modell erlaubte robuste Korrelationen zwischen Klinik und DTI-Daten aufzuzeigen und kann für zukünftige Studien empfohlen werden.

\section{P 19 Sind Neurofilamente geeignete Biomarker in spinaler Muskelatrophie (SMA)?}

Autoren Martakis $\mathrm{K}^{1}$, Wisch $\mathrm{H}^{1}$, Wurster $\mathrm{C}^{2}$, Claudi $\mathrm{K}^{1}$, Matthes $\mathrm{C}^{1}$,

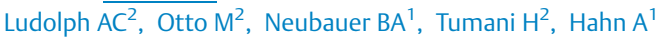

Institutes 1 Justus-Liebig Universität Giessen, UKGM, Neuropädiatrie, Gießen,

Deutschland; 2 Universität Ulm, Neurologie, Ulm, Deutschland

DOI 10.1055/s-0041-1723694

Hintergrund Neurofilament-light (Nf-L) wurde als Biomarker für neurodegenerative Erkrankungen vorgeschlagen. Ziel der Studie war es zu analysieren, wie sich Nf-L bei verschiedenen SMA-Typen verändert und wie die Nf-L in Liquor unter Nusinersen sich ändern.

Methode Mittels Enzymimmunoassay haben wir Nf-L in Liquor von Patienten mit SMA (Typ I: $\mathrm{n}=18$, II: $\mathrm{N}=32$ und III: $\mathrm{N}=8$ ) im Alter von 0 bis $33 \mathrm{~J}$ zu Studienbeginn sowie unter Nusinersen bestimmt. Die Basisdaten wurden mit 44 Kontrollen verglichen.

Ergebnisse Der Baseline Nf-L-Wert war bei 34 Patienten (59\%) höher als in der Kontrollgruppe ( $p<0,001)$. SMA-I-Patienten zeigten höhere Werte als SMA II und III ( $p<0,05)$. SMA-II-Patienten hatten höhere Werte als die Kontrollgruppe $(p<0,05)$. NF-L zwischen SMA-III- und Kontrollgruppe unterschieden sich nicht signifikant. Die NF-L-Werte waren nach Nusinersen (6 Mon) signifikant niedriger für das gesamte Patientengut im Vergleich zum Ausgangswert. Die NF-L nahmen in der SMA II-Gruppe signifikant ab, nicht jedoch in der SMA III-Gruppe. Wir sahen eine Senkung der NF-L-Werte bei Patienten mit SMA I unter Nusinersen. Die NF-LWerte zu Studienbeginn waren bei Patienten mit SMA I mit Krankheitsdauer von länger als 6 Monaten niedriger als bei Patienten mit kurzer Krankheitsdauer.

Schlussfolgerung NF-L ist als Biomarker für SMA hauptsächlich geeignet. SMATyp und Krankheitsdauer sollen bei der Interpretation der Konzentrationen und des Ansprechens auf die Behandlung berücksichtigt werden.

\section{P 20 Novel circulating Biomarkers may serve as sensitive outcome measures in Charcot-Marie-Tooth Disease (CMT1A)}

Authors Linhoff $\mathrm{L}^{1,2}$, Prukop $\mathrm{T}^{1,2}$, Leha $\mathrm{A}^{3}$, Sonja $\mathrm{F}^{4}$, Akova-Öztürk $\mathrm{E}^{5}$, Dräger $B^{5}$, Schlotter-Weigel $B^{6}$, Thiele $S^{6}$, Garcia-Angarita $N^{6}$, Greckl E6 Reinecke $L^{1}$, Rossner $M J^{6}$, Walter $M C^{6}$, Young $\mathrm{P}^{7}$, Sereda $M \mathrm{WW}^{8}$

Institute 1 University Medical Centre Göttingen, Department of Neurology, Göttingen, Deutschland; 2 Max-Planck-Institute for Experimental Medicine, Department of Neurogenetics, Göttingen, Deutschland; 3 University Medical Centre Goettingen, Göttingen, Deutschland; 4 Asklepios Fachklinikum, Psychiatrie, Tiefenbrunn, Deutschland; 5 University Hospital Muenster, Münster, Deutschland; 6 Ludwig-Maximilians University Munich, München, Deutschland; 7 Medical Park Bad Feilnbach Reithofpark, Bad Feilnbach, Deutschland; 8 University Medial Centre Göttingen, Department of Neurology, Göttingen, Deutschland

DOI 10.1055/s-0041-1723695

Introduction Charcot-Marie-Tooth disease 1A (CMT1A) is the most common inherited neuropathy caused by a duplication of the gene encoding PMP22. CMT1A is characterized by slow disease progression and a high variability, even among twins. The cause of disease variability is largely unknown. Previously, we could identify skin-derived disease and progression biomarkers in a rat model of CMT1A ("CMT rats"), which were translated to patients and validated in a large European and US-based cohort.

Material/Methods In a transcriptomic approach applying RNA sequencing in five mildly and five severely affected CMT1A patients and the CMT rat (WT vs. $M A$ vs SA $n=5$ ), we screened for candidates of circulating biomarkers We are currently validating these candidates in blood of 139 patients with CMT1A within the German CMT Disease Network (CMT-NET) and 27 healthy controls. Results We could validate overlapping candidates for CMT rats and CMT1A patient demonstrating feasibility of this translational approach. Differences in expression in a selected group of candidates were shown to correlate to clinical outcome measures (e.g. CMTNSv2) and were also sensitive to change over time. Discussion Clinically relevant, we could identified novel candidates from blood in both CMT rats and CMT1A patients that may serve as easily accessible novel biomarkers. These findings may serve as more sensitive outcome measures in clinical trials as well as disease course prediction in the clinic.

\section{P 21 Evaluation von Schluckstörungen bei Patienten mit Myotoner Dystrophie mittels Echtzeit-MRT (real- time MRI)}

Authors Zeng $\mathrm{R}^{1}$, Carstens $\mathrm{PO}^{1}$, Schütz $\mathrm{S}^{1}$, Olthoff $\mathrm{A}^{2}$, Weidenmüller $\mathrm{M}^{2}$,

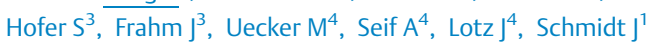

Institute 1 Universitätsmedizin Göttingen, Klinik für Neurologie, Göttingen, Deutschland; 2 Universitätsmedizin Göttingen, Klinik für Hals-NasenOhrenheilkunde, Phoniatrie und Pädaudiologie, Göttingen, Deutschland; 3 Max-Planck-Institut für Biophysikalische Chemie, Biomedizische NMR, Göttingen, Deutschland; 4 Universitätsmedizin Göttingen, Institut für diagnostische und interventionelle Radiologie, Göttingen, Deutschland

DOI $10.1055 / s-0041-1723696$ 
Einleitung Patienten mit Myotoner Dystrophie Typ 1 (DM1) sind häufig von einer Schluckstörung, Dysphagie, betroffen, welche zu Komplikationen wie Mangelernährung und Aspiration führen kann. Patienten mit Myotoner Dystrophie Typ 2 (DM2) können ebenfalls eine milde Dysphagie aufweisen. EchtzeitMRT ermöglicht die Untersuchung dynamischer Prozesse im Körper mit hoher zeitlicher Auflösung. Ziel dieser Studie ist die Evaluation und Charakterisierung von Schluckstörungen bei Patienten mit Myotoner Dystrophie mittels Echtzeit-MRT.

Material/Methode Bei Patienten mit DM1 und 2 sowie gesunden Kontrollprobanden wurde der Schluckakt mittels Echtzeit-MRT und fiberendoskopischer Schluckuntersuchung (FEES) untersucht. Die Ergebnisse der drei Gruppen wurden untereinander verglichen, sowie mit den Ergebnissen Patienten-basierter Fragebögen hinsichtlich Schluckstörungen korreliert.

Ergebnisse Die Echtzeit-MRT-Untersuchung zeigt eine signifikant verlängerte orale Transportzeit und eine verminderte Mundbodenverkürzung bei Patienten mit DM1, während Patienten mit DM2 keine signifikanten Unterschiede zu den gesunden Kontrollen zeigen.

Diskussion Eine Echtzeit-MRT Untersuchung ermöglicht die Identifikation krankheitsspezifischer Muster bei myogenen Dysphagien. Weitere Studien werden das Schluckmuster auch bei anderen neuromuskulären Erkrankungen untersuchen, um die zukünftige Diagnostik einer Schluckstörung hinsichtlich Qualität und Sensitivität zu verbessern.

\section{P 22 Predictors for myasthenic crisis in a myasthenia gravis cohort study}

Authors Nelke $\mathrm{C}^{1,2}$, Pawlitzki $\mathrm{M}^{1,2}$, Ruck $\mathrm{T}^{1,2}$

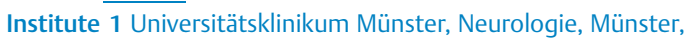

Deutschland; 2 Universitätsklinikum Münster, Institut für Neurologie mit

Institut für translationale Neurologie, Münster, Deutschland

DOI 10.1055/s-0041-1723697

Introduction Despite diagnostic and therapeutic advances for the management of myasthenia gravis (MG), patients experiencing myasthenic crisis (MC) continue to face a substantial mortality rate of approximately $5 \%$. The associated burden of disease underlines the importance for the prevention and management of MC. Our study provides analysis of predictors capable of identifying patients at risk for the occurrence of MC.

Methods We performed a retrospective, multicentre cohort study of $340 \mathrm{MG}$ patients. $95 \mathrm{MC}$ were recorded. We assessed the hazard ratio (HR) of sex, age, anti-Musk-antibody, Besinger score and MGFA status at diagnosis, presence of a thymoma using a multivariate Cox regression model with follow-up time as time variable. Experiencing at least one MC compared to no event was assessed as status variable.

Results Disease severity at diagnosis as indicated by Besinger score (HR 1.09 Cl $1.02-1.17 p=0.01$ ) or MGFA status (HR 1.96, Cl $1.25-3.06 p=0.003)$, the presence of a thymoma ( $\mathrm{HR} 2.03 \mathrm{Cl} 1.15-3.58 \mathrm{p}=0.03)$ and anti-Muskantibodies (HR $3.52 \mathrm{Cl} 1.66-7.45 \mathrm{p}=0.001)$ were independent predictors for experiencing MC during the individual course of disease.

Discussion The present study indicates that disease severity at diagnosis is a novel and independent risk factor for MC during disease. Early identification of patients at risk for $\mathrm{MC}$ by readily available clinical assessments might instruct clinicians to identify patients requiring intensified care.

\section{P 23 Thrombozytäre Granulasekretionsstörung bei Muskeldystrophie Duchenne - Ergebnisse einer prospektiven Diagnostikstudie}

Autoren Müller $C K^{1}$, Schorling $D C^{1}$, Pechmann $A^{1}$, Borell $S^{1}$, Rosenfelder $S^{2}$, Kölbel $\mathrm{H}^{3}$, Schara-Schmidt $\mathrm{U}^{3}$, Zieger $\mathrm{B}^{2}$, Kirschner $\mathrm{J}^{4}$

Institutes 1 Klinik für Neuropädiatrie und Muskelerkrankungen der ZKJ Freiburg, Freiburg, Deutschland; 2 Klinik für Pädiatrische Hämatologie und Onkologie des ZKJ Freiburg, Deutschland; 3 Abteilung für Neuropädiatrie,
Entwicklungsneurologie und Sozialpädiatrie des Universitäts-Klinikum Essen, Deutschland; 4 Abteilung für Neuropädiatrie des Zentrum für Kinderheilkunde Bonn, Deutschland

DOI 10.1055/s-0041-1723698

Hintergrund Patienten mit Muskeldystrophie Duchenne (DMD) zeigen bei Skoliose-OPs erhöhte perioperative Blutverluste. In mehreren Arbeiten konnte eine unauffällige plasmatische Gerinnung, aber eine verlängerte Blutungszeit bei DMD-Patienten gezeigt werden. Die Dystrophin-Isoform dp71 wird thrombozytär exprimiert und ist am Prozess der BlutplättchenKontraktion beteiligt.

Methode Bizentrische, nicht-verblindete, prospektive Diagnostikstudie bei 53 DMD-Patienten (mittleres Alter 14,5 Jahre [6;28]). Die Thrombozytenfunktion wurde durch Aggregometrie und Durchflusszytometrie (FACS) charakterisiert. Zusätzlich wurde die Funktion des von-Willebrand Faktors (vWF) untersucht (vWF-Antigen, vWF-Kollagenbindungskapazität, Multimerenstrukturanalyse).

Ergebnisse Es konnte eine reduzierte Aggregation bei Stimulation mit ADP in der Aggregometrie gezeigt werden (60\%; Referenzbereich 66-84\%). In der FACS zeigte sich eine signifikant erniedrigte Expression von CD62 und CD63. Beides sind Marker für die thrombozytäre Granulasekretion im Rahmen der Thrombozyten-Aktivierung. Die Untersuchung der anderen Teilschritte sowie des vWF war unauffällig.

Schlussfolgerung Die Ergebnisse legen eine thrombozytäre Granulasekretionsstörung bei DMD-Patienten nahe, welche ursächlich für die beobachtete Blutungsneigung sein kann. Ob es sich dabei um eine krankheitsspezifische Störung handelt oder ob eine sekundäre Entstehung vorliegt, kann durch diese Studie nicht beantwortet werden.

\section{P 24 Increased peripheral serum BDNF levels in patients with myasthenia gravis}

Authors Müller $\mathrm{P}^{1,2}$, van Wickeren $\mathrm{N}^{1,2}$, Klaus $\mathrm{B}^{1,2}$, Dordevic $\mathrm{M}^{1,2}$, Schmicker $\mathrm{M}^{1}$, Vielhaber $\mathrm{S}^{1,2,3}$, Brigadski $\mathrm{T}^{4}$, Lessmann $\mathrm{V}^{3,5}$, Schreiber $\mathrm{S}^{1,2,3}$, Müller $N^{1,2,3}$

Institute 1 Deutsches Zentrum für Neurodegenerative Erkrankungen, AG Neuroprotektion, Magdeburg, Deutschland; 2 Universitätsklinikum Magdeburg, Klinik für Neurologie, Magdeburg, Deutschland; 3 Center for behavioral brain sciences (CBBS), Magdeburg, Deutschland; 4 University of Applied Sciences, Department of Informatics and Microsystem Technology, Kaiserslautern, Deutschland; 5 Otto-von-Guericke Universität, Institut für Physiologie, Magdeburg, Deutschland

DOI 10.1055/s-0041-1723699

Introduction Myasthenia gravis (MG) is an autoimmune, neuromuscular diseases with autoantibodies targeting the postsynaptic membrane at the neuromuscular junction. MG causes muscle fatigue and is associated with systemic inflammation and cognitive deficits ${ }^{1}$. Here, we tested whether the latter may be related to changes in the levels of the brain-derived neurotrophic factor (BDNF). BDNF is substantial for neuronal development, synaptic plasticity, as well as for neuroprotection.

Materials \& Methods Ten MG patients (age: $64.7 \pm 16.1$; 7 male) were compared to age-, sex- and education-matched healthy controls (age: $64.7 \pm 15.9$ ). All patients received cholinesterase inhibitors. Plasma and serum concentrations of BDNF were determined from fasting blood samples using sandwich ELISAs (BDNF DuoSet; R\&D Systems, Wiesbaden, Germany).

Results MG patients showed significant higher BDNF serum $(p=.005, d=1.32)$ and a trend for higher BDNF plasma levels $(p=.075, d=0.77)$. However, no significant correlations between MG disease duration and BDNF levels was revealed. Discussion Our results indicate that MG is associated with increased BDNF serum levels. Potential mechanisms of enhanced BDNF levels could be a BDNF synthesis of (neuro-)inflammatory cells and/or drug treatment with cholinesterase inhibitors which may serve a neuroprotective role during inflammation ${ }^{2}$.

References [1] Gilhus, N. E. et al. doi: 10.1038/s41572-019-0079-y

[2] Molin, C. J. et al. doi: 10.1038/srep39716 
Krankheitsverlauf

\section{P 25 Hochdosis-Ivlg-Therapie bei chronisch inflammatorischer demyelinisierender Polyneuropathie (CIDP) unter Serum-IgG-Spiegel Kontrolle - ein Fallbericht}

\author{
Autoren Meyer $S^{1}$, Kummer $K^{1}$, Pickrodt $K^{1}$, Zechel $S^{2}$, Pauli $S^{3}$, Schmidt $J^{1}$, \\ Zschüntzsch J
}

Institutes 1 Universitätsmedizin Göttingen, Klinik für Neurologie, Göttingen, Deutschland; 2 Universitätsmedizin Göttingen, Institut für Neuropathologie, Göttingen, Deutschland; 3 Universitätsmedizin Göttingen, Institut für Humangenetik, Göttingen, Deutschland DOI 10.1055/s-0041-1723700

Hintergrund Die CIDP zählt zu den autoimmunvermittelten Polyneuropathien (PNP). Eine zugelassene und wirksame Therapie ist die Gabe von intravenösen Immunglobulinen (Ivlg). Wir präsentieren einen nach EFNS-Kriterien diagnostizierten CIDP-Patienten, der auf Standardtherapien inkl. der allgemein empfohlenen Ivlg-Dosierung mit 1-2g/kg KG kein Ansprechen zeigte.

Methoden IgG-Spiegel im Serum, molekulargenetische PNP-Panel-Diagnostik, ENG, Nervensonographie

Ergebnisse Die Vorstellung des Pat. erfolgte nach einem Wohnortwechsel und einer Ivlg-Infusionsverzögerung mit Hypästhesien und einer progredienten Tetraparese bis hin zur Bettlägerigkeit. Korrelierend bestanden CIDP-typische Veränderungen in der apparativen Diagnostik. Der Pat. war bereits zuvor extern mit hochdosierten Ivlg-Gaben alle 4-8 Wochen behandelt worden, da es unter Kortikoiden, Rituximab und Plasmapherese sowie unter niedrigen Ivlg-Dosierungen zu einer Symptomverschlechterung gekommen war. Eine Dosis von $800 \mathrm{~g}$ IvIG pro Monat führte zu einer Verbesserung. Die Erhaltungsdosis wurde unter Kontrolle des Serum-IgG-Spiegels auf 140-160g Ig i.v. und s.c./Woche eingestellt.

Diskussion Einzelne Patienten mit CIDP profitieren von einer Ivlg - Dosierung oberhalb der o.g. Empfehlung. Vor einem Therapiewechsel ist im individuellen Fall die Bestimmung des Serum-IgG-Spiegels sinnvoll. Die Ursachen des divergierenden Ansprechens können in einer verstärkten Metabolisierung der I oder einem Fc-Rezeptor-Polymorphismus liegen.

\section{P 26 Langzeitverlauf älterer Patienten mit late-onset Pompe disease (LOPD) unter Enzymersatztherapie (enzyme replacement therapy, ERT)}

Autoren Winkler $\mathrm{M}^{1}$, von Landenberg $\mathrm{C}^{1}$, Reimann $]^{1}$, Kornblum $\mathrm{C}^{1}$ Institute 1 Universitätsklinikum Bonn, Klinik für Neurologie, Sektion Neuromuskuläre Erkrankungen, Bonn, Deutschland DOI 10.1055/s-0041-1723701

Erste Studien zum Langzeitverlauf einer Therapie mit Alglucosidase $\alpha$ bei LOPD zeigen einen max. Benefit in den ersten Jahren mit meist sekundärem decline. Wir zeigen die Effekte einer Langzeit-ERT in einer älteren LOPD-Kohorte. Einschlusskriterien waren Alter > $50 \mathrm{~J}$. bei Diagnose und ERT-Beginn sowie ERTDauer $>7 \mathrm{~J}$. Outcomeparameter waren MRC sum-score, 6-minute walk test 6MWT, Quick Motor Function Test QMFT, forcierte Vitalkapazität im Sitzen und Liegen (FVC), CK Werte und IgG Antikörper (AK)-Titer gegen Alglucosidase $\alpha .6$ Patienten wurden eingeschlossen (3f); Altersmedian bei Symptombeginn $49 \mathrm{~J}$. (range 40-64) und Alter bei Diagnose und ERT-Beginn 63 J. (52-69). Die mittlere ERT-Dauer war 8 J. (7-12). Es bestand keine Assoziation zwischen Outcome, Mutationskonstellation, myohistopathologischen Veränderungen, Enzymaktivität und AK-Titern. Die Höhe der AK-Titer korrelierte nicht mit Dosis und Ansprechen der ERT. Initial profitierten > 50\% der Patienten von der ERT (6MWT, FVC, MRC sum-score). Alle zeigten im Verlauf eine Verschlechterung der FVC im Sitzen, während 33 \% eine Stabilisierung/Verbesserung der FVC im Liegen aufwiesen. $33 \%$ erreichten eine Stabilisierung/Verbesserung der Muskelkraft, aber eine Verschlechterung im QMFT bei $83 \%$ im Verlauf. $67 \%$ profitierten langfristig im 6MWT. Die besten Ergebnisse waren im 6MWT, FVC im Liegen und MRC sum score zu sehen.

Hier konnte bei heterogenen Effekten insg. ein Benefit in der älteren LOPDKohorte nachgewiesen werden.

\section{P 27 Inzidenz des postpunktionellen Syndroms bei Patienten mit SMA unter Therapie mit Nusinersen}

Autoren Schorling $D C^{1}$, Pechmann $A^{1}$, Eckenweiler $\mathrm{M}^{1}$, Müller $C^{1}$, Langer $\mathrm{T}^{1}$, Kirschner $]^{2}$

Institutes 1 Zentrum für Kinder- und Jugendmedizin, Medizinische Fakultät,

Universitätsklinikum Freiburg, Klinik für Neuropädiatrie und

Muskelerkrankungen, Freiburg, Deutschland; 2 Universitätsklinikum Bonn,

Klinik für Neuropädiatrie, Bonn, Deutschland

DOI 10.1055/s-0041-1723702

Fragestellung Obwohl das postpunktionelle Syndrom (engl. post-dural puncture headache, PDPH) eine häufige Komplikation nach Lumbalpunktionen (LP) darstellt, existieren nur wenige epidemiologische Daten zum Auftreten nach therapeutischen Lumbalpunktionen bei Patienten mit Spinaler Muskelatrophie (SMA).

Methoden Retrospektive Analyse zum Auftreten von postpunktionellen Beschwerden bei SMA-Patienten und einer allgemeinpädiatrischen Kontrollgruppe. Patientenkurven und die SMArtCARE Dokumentationsbögen wurden hinsichtlich der Dokumentation von Kopfschmerzen, die die PDPH-Diagnosekriterien der IHS erfüllen, überprüft. Nicht-SMA Patienten erhielten zusätzliche Fragebogen.

Ergebnisse Es wurden 196 LPs bei 26 SMA-Patienten identifiziert (Typ 1=3; Typ2=7; Typ 3=16; mittleres Alter 11,5 Jahre). Die IHS-Diagnosekriterien waren nach 4,6\% aller LPs erfüllt (9 LPs bei 7 Patienten; mittleres Alter 15 Jahre). Demgegenüber lag die Inzidenz der pädiatrischen Kontrollgruppe (129 LPs bei 116 Patienten; mittleres Alter 11,7 Jahre) bei 13.2\% (17 LPs bei 16 Patienten; mittleres Alter 14,7 Jahre).

Schlussfolgerungen Die PDPH-Inzidenz nach wiederholten therapeutischen LPs in unserer SMA-Kohorte war niedriger als die PDPH-Prävalenz in der allgemeinpädiatrischen Kohorte. Da das abgenommene Liquorvolumen durch Nusinersen-Gabe (12mg in $5 \mathrm{ml}$ Trägerlösung) ersetzt wird, scheinen weniger ausgeprägte Druckschwankungen ursächlich für die beobachtete Differenz.

\section{P 28 Versorgungssituation von ALS-Patienten in den letzten Lebensmonaten - die Sicht der Angehörigen}

Autoren Linse $\mathrm{K}^{1,2}$, Aust $\mathrm{E}^{1}$, Günther $\mathrm{R}^{1,2}$, Hermann $\mathrm{A}^{3,4}$

Institutes 1 Technische Universität, Abteilung Neurologie, Dresden,

Deutschland; 2 Deutsches Zentrum für Neurodegenerative Erkrankungen, Dresden, Deutschland; 3 Universität Rostock, Translationale

Neurodegeneration Sektion “Albrecht Kossel”, Abt. Neurology, Rostock, Deutschland; 4 Deutsches Zentrum für Neurodegenerative Erkrankungen,

Standort Rostock/Greifswald, Deutschland

DOI 10.1055/s-0041-1723703

Einleitung Amyotrophe Lateralsklerose (ALS) führt zu einer zunehmenden Abhängigkeit von Hilfsmitteln und Unterstützung, welche zu großen Teilen durch Angehörige erbracht wird. Die komplexe Behandlungsplanung bis zum Lebensende erfordert entsprechend internationaler Standards eine qualifizierte (palliativ-)medizinische Begleitung.

Methoden In Telefoninterviews mit 47 Angehörigen verstorbener ALS-Patienten wurden Daten zur Versorgungssituation, Sterbephase und Angehörigenbelastung (HSP-k) erhoben.

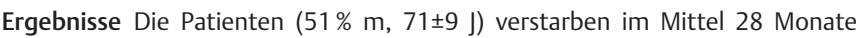
nach Diagnosestellung mit einem ALSFRS-R-Score von 14 (Range 0-30). Die Angehörigen ( $40 \%$ m, 65 \pm 13 J, 66\% Ehepartner) pflegten im Mittel 7 Stunden am Tag. Häufig berichtete Probleme betrafen die Hilfsmittelversorgung (47\%) und die Versorgung durch Pflegeheime bzw. -dienste (32\%). In $46 \%$ der Fälle waren am Lebensende palliativmedizinische Akteure involviert -alle diese 
Patienten verstarben "friedlich" und am gewünschten Ort. Die Angehörigenbelastung war sehr variabel (51\% mäßig, $7 \%$ hoch), nur $15 \%$ erhielten vor oder nach dem Tod des Patienten psychologische Unterstützung, 56 \% erlitten subjektiv teils dauerhafte gesundheitliche Schäden (seelisch/körperlich).

Schlussfolgerungen Eine Verbesserung der Versorgung von ALS-Patienten und deren Angehörigen hinsichtlich Hilfsmitteln und Palliation bis ans Lebensende ist dringend notwendig, auch zur Reduktion von Belastung und Folgeschäden seitens der Angehörigen.

\section{P 29 Langzeitverlauf eines 13-jährigen Patienten mit schwerem Freeman-Sheldon-Syndrom}

\author{
Autoren Rudnik-Schöneborn $\mathrm{S}^{1}$, Baumann $\mathrm{M}^{2}$ \\ Institutes 1 Institut für Humangenetik, Medizinische Universität Innsbruck, \\ Innsbruck, Österreich; 2 Universitätsklinik, Kinderheilkunde, Innsbruck, \\ Österreich
}

DOI 10.1055/s-0041-1723704

Das Freeman-Sheldon-Syndrom (FSS) gehört zu den distalen Arthrogryposen und wird auch als "whistling face"-Syndrom bezeichnet. Merkmale sind Fehlstellungen von Händen und Füßen, eine schwere Skoliose, Ernährungs- und Lungenfunktionsstörungen sowie faziale Kontrakturen. Bei über $90 \%$ aller Patienten liegt eine heterozygote Mutation im MYH3-Gen vor, von denen die p. Thr178Ile-Mutation die schwerste und bisher nur als de novo Mutation beschrieben ist.

Wir stellen einen 13-jährigen Knaben mit FSS aufgrund einer de novo p. Thr178lle-Mutation vor. Intrauterin bestanden ein Polyhydramnion, Hand- und Fußfehlstellungen sowie eine Mikrostomie. Postnatal wurde das Kind intubiert und mit wenigen Monaten tracheotomiert. Die Finger waren in Windmühlenstellung, die Füße in Klumpfußstellung eingeschlagen. Nach zahlreichen Korrekturoperationen hat der Patient mit etwa 5 Jahren gehen gelernt. Er ist mit Unterschenkel-Orthesen gehfähig und nutzt für längere Strecken einen Rollator. Es bestehen eine Hüftluxation und eine Skoliose. Er ist kleinwüchsig (91 cm, -8 SD, 12,7 kg, -8 SD) und entspricht in den Maßen einem 2,5 Jahre (!) alten Kind. Trotz fazialer Kontrakturen gelingt das Sprechen gut über einen Tracheostoma-Aufsatz. Er wird nachts für 10 Stunden heimbeatmet. Seine kognitive Entwicklung verläuft unauffällig.

Langzeitverläufe mit der p.Thr178lle-Mutation sind bisher nicht bekannt, unser Fallbericht soll zu einem besseren Verständnis für diese außerordentlich seltene Erkrankung beitragen.

\section{P 30 Individuelle gesundheitsbezogene Lebensqualität und gesamtgesellschaftliche Kosten bei amyotropher Lateralsklerose (ALS) unter Berücksichtigung unterschiedlicher Verlaufsformen}

Autoren Heinrich $F^{1}$, Cordts $I^{2}$, Günther $R^{3,4}$, Zeller $D^{5}$, Schischlevskij $P^{1}$,

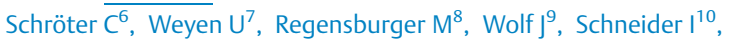
Hermann $A^{11}$, Metelmann $M^{12}$, Kohl $Z^{13}$, Linker $R^{13}$, Koch $J C^{14}$, Stendel $C^{15,16}$, Peseschkian $T^{1}$, Osmanovic $A^{1}$, Klopstock $T^{15,16,17}$, Dorst $\mathrm{J}^{18}$, Boentert $\mathrm{M}^{19,20}$, Hagenacker $\mathrm{T}^{21}$, Deschauer $\mathrm{M}^{2}$, Lingor $\mathrm{P}^{2}$, Petri $\mathrm{S}^{1}$, Schreiber-Katz $\mathrm{O}^{1}$

Institutes 1 Medizinische Hochschule Hannover, Klinik für Neurologie, Hannover, Deutschland; 2 Klinikum Rechts der Isar, Technische Universität München, Klinik und Poliklinik für Neurologie, München,

Deutschland; 3 Universitätsklinikum Carl Gustav Carus an der Technischen Universität Dresden, Klinik und Poliklinik für Neurologie, Dresden, Deutschland; 4 Deutsches Zentrum für neurodegenerative Erkrankungen (DZNE) Dresden, Deutschland; 5 Universitätsklinikum Würzburg, Neurologische Klinik und Poliklinik, Würzburg, Deutschland; 6 Klinik Hoher Meißner, Neurologische Abteilung, Bad Sooden-Allendorf, Deutschland; 7 Berufsgenossenschaftliches Universitätsklinikum Bergmannsheil gGmbH, Neurologische Universitätsklinik und Poliklinik,
Bochum, Deutschland; 8 Universitätsklinikum Erlangen, MolekularNeurologische Abteilung, Erlangen, Deutschland; 9 Diakonissenkrankenhaus Mannheim, Neurologische Klinik, Mannheim, Deutschland; 10 Martin-LutherUniversität Halle-Wittenberg, Klinik und Poliklinik für Neurologie, Halle a. d. S., Deutschland; 11 Universitätsmedizin Rostock, Sektion für Translationale Neurodegeneration "Albrecht Kossel”, Klinik und Poliklinik für Neurologie, Rostock, Deutschland; 12 Universitätsklinikum Leipzig, Klinik und Poliklinik für Neurologie, Leipzig, Deutschland; 13 Universität Regensburg, Klinik und Poliklinik für Neurologie am medbo Bezirksklinikum Regensburg, Regensburg, Deutschland; 14 Universitätsmedizin Göttingen, Klinik für Neurologie, Göttingen, Deutschland; 15 LMU Klinikum München, Friedrich-Baur-Institut an der Neurologischen Klinik und Poliklinik, München, Deutschland; 16 Deutsches Zentrum für neurodegenerative Erkrankungen (DZNE), München,

Deutschland; 17 Munich Cluster for Systems Neurology (SyNergy), München, Deutschland; 18 RKU- Universitäts- und Rehabilitationskliniken Ulm, Neurologische Universitätsklinik, Neuropsychologie, Ulm, Deutschland; 19 Universitätsklinikum Münster, Klinik für Neurologie mit Institut für Translationale Neurologie, Münster, Deutschland; 20 UKM Marienhospital Steinfurt, Klinik für Innere Medizin, Steinfurt, Deutschland;

21 Universitätsklinikum Essen (AöR), Klinik für Neurologie, Essen, Deutschland DOI 10.1055/s-0041-1723705

Einleitung Die ALS verläuft meist innerhalb weniger Jahre rasch progredient und verursacht deutliche Einschnitte in der Lebensqualität der Patienten und hohe Kosten. Die beiden Hauptmanifestationsformen (bulbär vs. spinal) unterscheiden sich im Verlauf. In dieser Studie werden Auswirkungen der ALS-Form auf Lebensqualität und Krankheitskosten aus einer gesellschaftlichen Perspektive untersucht.

Methoden In einer deutschlandweiten, multizentrischen Querschnittsstudie wurden im Zeitraum 08/18-03/20 Patienten mit verschiedenen Motoneuronerkrankungen eingeschlossen und mittels standardisierter Fragebögen zu Symptomlast, Erkrankungskosten und Lebensqualität befragt. Daraus wurden gesamtgesellschaftliche Kosten berechnet und in Bezug zur Lebensqualität (EQ-5D-5L ${ }^{\mathrm{TM}}-\mathrm{Index}$ ) gesetzt. Die hier vorgestellte Fragestellung ist ein Teilaspekt dieser Studie.

Ergebnisse In die Studie wurden 325 Patienten mit ALS eingeschlossen, davon $70,2 \%$ mit spinalem Beginn. Der mittlere Lebensqualitätsindex war bei der spinalen Form um 0,20 kleiner als bei bulbärer Form. Bei der spinalen Form sind höhere gesamtgesellschaftliche Kosten (im Mittel 85730€/Jahr/Patient) zu beobachten, dies ist unter anderem durch einen Anstieg indirekter Kosten (u.a. Arbeitskraftverluste) bedingt.

Diskussion In der Entwicklung neuer Therapie- und Versorgungsansätze für die ALS ist es wichtig, die Erkrankungsformen differenziert zu betrachten, um gezielt Lebensqualität verbessern und Krankheitskosten senken zu können.

\section{P 31 A comparison of the EuroQoL 5-Dimension 5- Level Questionnaire (EQ-5D-5L) and the Amyotrophic Lateral Sclerosis Assessment Questionnaire (ALSAQ-5) assessing health-related quality of life in amyotrophic lateral sclerosis (ALS) patients in Germany.}

Authors Peseschkian $T^{1}$, Cordts $I^{2}$, Günther $R^{3,4}$, Zeller $D^{5}$, Heinrich $F^{1}$, Schröter $\overline{C^{6} \text {, Weyen } U^{7}}$, Regensburger $M^{8}$, Wolf $\rfloor^{9}$, Schneider $I^{10}$, Hermann $A^{11}$, Metelmann $M^{12}$, Kohl $Z^{13}$, Linker $R^{13}$, Koch $J C^{14}$, Büchner $B^{15}$, Schischlevskij $P^{1}$, Osmanovic $A^{1}$, Klopstock $T^{15,16,17}$, Dorst $]^{18}$, Boentert $\mathrm{M}^{19,20}$, Hagenacker $\mathrm{T}^{21}$, Deschauer $\mathrm{M}^{2}$, Lingor $\mathrm{P}^{2}$, Petri $\mathrm{S}^{1}$, Schreiber-Katz $\mathrm{O}^{1}$

Institute 1 Medizinische Hochschule Hannover, Klinik für Neurologie, Hannover, Deutschland; 2 Klinikum Rechts der Isar, Technische Universität München, Klinik und Poliklinik für Neurologie, München, Deutschland; 3 Universitätsklinikum Carl Gustav Carus an der Technischen Universität Dresden, Klinik und Poliklinik für Neurologie, Dresden, Deutschland; 4 Deutsches Zentrum für neurodegenerative Erkrankungen (DZNE), Dresden, Deutschland; 5 Universitätsklinikum Würzburg, Neurologische Klinik und 
Poliklinik, Würzburg, Deutschland; 6 Klinik Hoher Meißner, Neurologische Abteilung, Bad Sooden-Allendorf, Deutschland; 7 Berufsgenossenschaftliches Universitätsklinikum Bergmannsheil gGmbH, Neurologische Universitätsklinik und Poliklinik, Bochum, Deutschland; 8 Universitätsklinikum Erlangen, Molekular-Neurologische Abteilung, Erlangen,

Deutschland; 9 Diakonissenkrankenhaus Mannheim, Neurologische Klinik, Mannheim, Deutschland; 10 Martin-Luther-Universität Halle-Wittenberg, Klinik und Poliklinik für Neurologie, Halle a. d. S.,

Deutschland; 11 Universitätsmedizin Rostock, Sektion für Translationale Neurodegeneration “Albrecht Kossel”, Klinik und Poliklinik für Neurologie, Rostock, Deutschland; 12 Universitätsklinikum Leipzig, Klinik und Poliklinik für Neurologie, Leipzig, Deutschland; 13 Universität Regensburg, Klinik und Poliklinik für Neurologie am medbo Bezirksklinikum Regensburg, Regensburg, Deutschland; 14 Universitätsmedizin Göttingen, Klinik für Neurologie, Göttingen, Deutschland; 15 LMU Klinikum München, Friedrich-Baur-Institut an der Neurologischen Klinik und Poliklinik, München, Deutschland; 16 Deutsches Zentrum für neurodegenerative Erkrankungen (DZNE), München,

Deutschland; 17 Munich Cluster for Systems Neurology (SyNergy), München, Deutschland; 18 RKU- Universitäts- und Rehabilitationskliniken Ulm,

Neurologische Universitätsklinik, Neuropsychologie, Ulm, Deutschland; 19 Universitätsklinikum Münster, Klinik für Neurologie mit Institut für Translationale Neurologie, Münster, Deutschland; 20 UKM Marienhospital Steinfurt, Klinik für Innere Medizin, Steinfurt, Deutschland;

21 Universitätsklinikum Essen (AöR), Klinik für Neurologie, Essen, Deutschland DOI 10.1055/s-0041-1723706

Introduction Improving the quality of life (QoL) of patients is central to ALS treatment. One established questionnaire measuring health-related quality of life (HRQoL) is the EQ-5D-5L. The ALSAQ-5 questionnaire was developed to measure ALS-specific HRQoL, though its German translation is not validated. The present study describes HRQoL in ALS and examines whether the ALSAQ-5 is robust enough to compare with the EQ-5D-5L in measuring HRQoL.

Methods In a Germany-wide, multicenter cross-sectional study in the period from 08/18-03/20, patients with ALS (defined after revised El Escorial criteria) were asked to complete a questionnaire that included QoL assessment. From this data, we analysed the results of questions (Q) 1, 2 and 5 of both questionnaires, as these had common subject matters. Furthermore, the ALSAQ-5 and EQ VAS (visual analogue scale) scores were compared.

Results 325 patients with ALS were included in the larger study. Of these, 288 filled in all five questions of the ALSAQ-5, whilst 283 filled in all five questions of the EQ-5D-5L. The following correlations were found: $\mathrm{Q} 1=0.72, \mathrm{Q} 2=0.69$, $\mathrm{Q} 5=0.54$. In addition, the correlation between the total ALSAQ-5 and EQ VAS score was 0.63 .

Discussion This study is a first step in showing that a correlation exists between the German-language ALSAQ-5 and the EQ-5D-5L. Having an easy to use bed-side test to evaluate ALS-specific QoL will allow for more focused and relevant treatment for patients.

\section{P 32 Adaptation einer psychotherapeutisch- supportiven Kurzzeitintervention für Patienten mit Amyotropher Lateralsklerose - eine Pilotstudie}

\footnotetext{
Autoren Metelmann $\mathrm{M}^{1}$, Esser $\mathrm{P}^{2}$, Peuker $\mathrm{M}^{2}$, Claßen J ${ }^{1}$, MehnertTheuerkauf $\mathrm{A}^{2}$

Institutes 1 Universitätsklinikum Leipzig, Klinik und Poliklinik für Neurologie, Leipzig, Deutschland; 2 Universitätsklinikum Leipzig, Abteilung für Medizinische Psychologie und Medizinische Soziologie, Leipzig, Deutschland DOI 10.1055/s-0041-1723707

Einleitung Patienten mit Amyotropher Lateralsklerose (ALS) haben bei fehlenden kurativen Behandlungsmöglichkeiten eine deutlich reduzierte Lebenserwartung. Damit geht neben der zunehmenden körperlichen Behinderung eine hohe psychosoziale Belastung einher.
}

Methoden Für diese Pilotstudie wurden Patienten mit einer möglichen, wahrscheinlichen oder sicheren ALS nach den revidierten El Escorial-Kriterien ( $n=5)$ eingeschlossen. Sie erhielten eine Kurzzeit-Intervention durch eine approbierte Psychotherapeutin über 6 Monate. Im Fokus der Studie standen Machbarkeit und Bewertung der Therapie durch die Patienten. Ausgewählte Zielgrößen wurden vor der Therapie (T0) und direkt im Anschluss (T1) erhoben.

Ergebnisse 15 Patienten wurden gescreent, von welchen 4 Patienten 6 bis 7 Therapieeinheiten erhielten. $22 \%$ der Einheiten erfolgten wegen progredienter Mobilitätseinschränkung telefonisch. Für den Zeitpunkt T1 lagen Antworten von 3 Patienten vor, die die Relevanz aller Therapiemodule mit $\geq 5$ Punkten auf einer 7-stufigen Likert-Skala (7=höchste Zufriedenheit) bewerteten.

Diskussion Bei den Teilnehmenden zeigte sich eine hohe Zufriedenheit mit der psychotherapeutischen Intervention. Jedoch war die Durchführung der Therapie über 6 Monate aufgrund zunehmender Einschränkung von Mobilität und Sprechfähigkeit erschwert. Zur Überprüfung der Wirksamkeit ist eine Anpassung des Studiendesigns mit stärkerer Berücksichtigung der durch die Krankheitsprogression bedingten Einschränkungen notwendig

\section{Krankheitsverlauf}

\section{P 33 Integration von Beatmungsmedizin, Rehabilitation und Palliativmedizin in einer interdisziplinären Ambulanz}

Autoren Groß $\mathrm{M}^{1}$

Institute 1 Evangelisches Krankenhaus Oldenburg, Neurologische

Intensivmedizin, Frührehabilitation, Palliativmedizin, Oldenburg, Deutschland DOI 10.1055/s-0041-1723708

Patienten mit schweren neuromuskulären Erkrankungen stellen komplexe Anforderungen an Ihre Behandler: Die Teilhabe ist durch eingeschränkte Mobilität und erschwerte Kommunkation beeinträchtigt. Störungen der Atmung, des Schluckens und Hustens sowie die resultierende Sekretretention können schwere, oft sogar lebensbedrohliche Symptome verursachen. Spastik, Schmerzen und Dyspnoe können zu erheblichem Leid führen. Auch die Angehörigen sind hohen Belastungen ausgesetzt, die durch Lücken im Sozialversicherungssystem oder kognitive Störungen der Patienten verstärkt werdenkönnen. Wir berichten über den patientenzentrierten, familienorientierten Einsatz der Ressourcen eines interdisziplinären Teams mit Ärzten verschiedener Fachdisziplinen, Atmungstherapeuten, Logopäden, Physiotherapeuten, Palliativpflegekräften und einer Fachkraft für Unterstützte Kommunikation. In der seit 2 Jahren bestehenden Ambulanz werden die Patienten und die Angehörigen beraten sowie beatmungsmedizinisch, rehabilitativ und palliativmedizinisch behandelt. Belastende stationäre Aufenthalte können so vermieden werden. Zwischen den Terminen steht die Ambulanz Patienten, Angehörigen, (Intensiv-)Pflegediensten und niedergelassenen ärztlichen Kollegen als Ansprechpartner zur Verfügung.

\section{P 34 Das traumatische Kiloh-Nevin-Syndrom als Folge eines Retroversionstraumas}

Autoren Kummer $\mathrm{K}^{1}$, Micheilis $\mathrm{T}^{1}$, Zschüntzsch j ${ }^{1}$

Institute 1 Universitätsmedizin Göttingen, Klinik für Neurologie, Göttingen, Deutschland DOI 10.1055/s-0041-1723709

Einleitung Das Kiloh-Nevin-Syndrom (KNS) ist gekennzeichnet durch eine Affektion des N. interosseus anterior (AIN) mit Ausfall des M. flexor pollicis longus und des M. flexor digitorum profundus des Indexfingers. Neben einem idiopathischen Auftreten sind entzündliche, traumatische, iatrogene und komprimierende Ursachen bekannt. In den meisten Fällen kommt es zu einer spontanen Besserung der Beschwerden. Selten werden operative Dekompressionen des AIN durchgeführt. 
Methoden Klinische, elektrophysiologische und sonographische Fallpräsentation von 3 Patienten mit KNS, welche denselben Unfallhergang einer ruckartigen Retroversion in Supinations- und leichter Abduktionsstellung des Armes beschrieben.

Ergebnis 1) 50-jährige Patientin mit temporal dispersem KNS bds. nachdem sie mit den Skistöcken hängengeblieben war.

2) 40-jähriger Patient mit inkomplettem KNS links 2 Tage nach dem Hochziehen seiner Kinder auf einen Hügel, mit reißenden Schulterschmerzen links in der ersten Nacht.

3) 35-jähriger Patient mit KNS bds. nach Sturz beim Wandern nach hinten und starken Unterarmschmerzen in den ersten 3 Tagen.

Bei allen Patienten zeigte sich sonographisch eine Schwellung des N. medianus im Bereich der Oberarme.

Diskussion Die präsentierten Fälle demonstrieren einen bislang nicht beschriebenen Mechanismus eines traumatischen KNS. Eine sorgfältige Anamnese, Untersuchung sowie eine Nervensonographie verkürzen die Zeit bis zur korrekten Diagnose und können unnötige Eingriffe verhindern.

Versorgung und Real-Life-Outcome

\section{P 35 Edaravone Therapie bei Amyotropher Lateralsklerose - Klinische Parameter und Erfahrungen aus einer multizentrischen Analyse von Real-World- Daten}

Autoren Witzel $S^{1}$, Maier $A^{2}$, Steinbach $R^{3}$, Grosskreutz $J^{3}$, Koch $J C^{4}$, Sarikidi $A^{5}$, Petri $S^{5}$, Günther $R^{6}$, Wolf $]^{7}$, Hermann $A^{8}$, Prudlo $\rfloor^{9}$, Cordts $I^{10}$, Lingor $\mathrm{P}^{10}$, Löscher $W N^{11}$, Kohl $Z^{12}$, Hagenacker $T^{13}$, Koch $B^{2}$, Spittel $S^{2}$, Günther $\mathrm{K}^{14}$, Michels $\mathrm{S}^{14}$, Dorst $\mathrm{J}^{14}$, Meyer $\mathrm{T}^{2}$, Ludolph $\mathrm{AC}^{14}$ Institutes 1 Universitäts- und Rehabilitationskliniken Ulm, Klinik für Neurologie, Ulm, Deutschland; 2 Charité - Universitätsmedizin Berlin, Ambulanz für ALS und andere Motoneuronenerkrankungen, Berlin, Deutschland; 3 Universitätsklinikum Jena, Klinik für Neurologie, Jena, Deutschland; 4 Universitätsklinikum Göttingen, Klinik für Neurologie, Göttingen, Deutschland; 5 Medizinische Hochschule Hannover, Klinik für Neurologie, Hannover, Deutschland; 6 Universitätsklinikum Carl Gustav Carus, Klinik für Neurologie, Dresden, Deutschland; 7 Diakonissenkrankenhaus Mannheim, Klinik für Neurologie, Mannheim, Deutschland;

8 Universitätsmedizin Rostock, Sektion für Translationale Neurodegeneration "Albrecht Kossel", Rostock, Deutschland; 9 Universitätsmedizin Rostock, Klinik für Neurologie, Rostock, Deutschland; 10 Klinikum rechts der Isar Technische Universität München, Klinik für Neurologie, München, Deutschland; 11 Medizinische Universität Innsbruck, Klinik für Neurologie, Innsbruck, Österreich; 12 Universität Regensburg, Klinik für Neurologie, Regensburg, Deutschland; 13 Universitätsklinikum Essen, Klinik für Neurologie, Essen, Deutschland; 14 Universitäts- und Rehabilitationskliniken Ulm, Neurologie, Ulm, Deutschland DOI 10.1055/s-0041-1723710

Einleitung Eine Phase-III-Studie zeigte für das i.v.-Medikament Edaravone eine Verlangsamung der Krankheitsprogression bei ALS. Kritikpunkte sind die Generalisierbarkeit der Ergebnisse durch stark selektierende Einschlusskriterien (efficacy expected subpopulation (EESP)-Kriterien) und das komplexe Therapieschema. Bei fehlender Arzneimittelzulassung erfolgte die Anwendung in Deutschland durch individuelle Heilversuche.

Methoden Diese multizentrische Studie wertete Daten von 194 mit Edaravone behandelten ALS-Patienten aus 12 Kliniken aus (06/2017-03/2020). Wir analysierten die Einhaltung der EESP-Kriterien, die Progressionsrate anhand des ALSFRS-R Abfalls vor und nach Therapiebeginn, Nebenwirkungen und die Behandlungszufriedenheit mittels TSQM-9.

Ergebnisse Bei Therapiebeginn erfüllten $8 \%$ der Patienten alle 6 EESP-Kriterien, $59 \%$ erfüllten 4 oder 5 . Das am häufigsten verfehlte EESP-Kriterium (79\%) waren $\geq 2$ Punkte in jedem ALSFRS-R Item. Unter Therapie war die Progressionsrate, verglichen mit den Monaten vor Therapiebeginn, in etwa konstant (Mediane Änderung: -0,09 ALSFRS-R-Punkte/Monat; $95 \% \mathrm{KI}$ : 0,12 bis -0,27). Trotz geringer Nebenwirkungsrate (16\%), brachen 51 Patienten (26\%) die Therapie ab. Die Zufriendenheit mit der Therapie insgesamt lag bei 59 von $100 \%$ (SD $\pm 32 \%$ ).

Diskussion Unsere Real-World-Daten bieten einen realistischen Blick auf die Edaravone-Behandlung und zeigen die Herausforderungen bei der praktischen Anwendung dieser kritisch diskutierten, neuen ALS-Therapie.

\section{P 36 X-chromosomale Myotubuläre Myopathie - 20 Jahre Nachbeobachtungszeit in einem Zentrum}

Autoren Gangfuß $A^{1}$, Schmitt $D^{2}$, Roos $A^{1}$, Braun $F^{1}$, Annoussamy $M^{3,4}$, Servais $\mathrm{L}^{5,6}$, Schara-Schmidt $U^{1}$

Institutes 1 Universitätsklinikum Essen, Kinderklinik I, Neuropädiatrie, Essen, Deutschland; 2 Audentes Therapeutics, San Francisco, CA, Vereinigte Staaten; 3 I-Motion, Institue de Myology, Paris, Frankreich; 4 Sysnav, Vernon, Frankreich; 5 University of Liège, Neuromuscular Disease Reference Center, Liège, Belgien; 6 MDUK Neuromuscular Center, Department of Pediatrics,

Oxford, Vereinigtes Königreich

DOI 10.1055/s-0041-1723711

Die X-chromosomale myotubuläre Myopathie (XLMTM) ist eine lebensbedrohliche seltene neuromuskuläre Erkrankung, die durch pathogene Varianten im MTM1-Gen verursacht wird. Sie weist eine große phänotypische Heterogenität auf. Diese reicht von Patienten, die unabhängig gehen können, bis zu immobilen Patienten, die nur in der Lage sind, die Hand zum Mund zu führen und 24 Stunden am Tag auf ein Beatmungsgerät angewiesen sind. Diese Tatsache macht deutlich, dass die Erfordernis eins Beatmungsgerätes nicht das vollständige klinische Bild von Patienten mit XLMTM veranschaulicht.

In dieser Studie haben wir die Daten von 13 deutschen XLMTM-Patienten, die über einen Zeitraum von bis zu 20 Jahren in unserer Universitätskinderklinik erhoben wurden, ausgewertet. Wir haben sie mit den Daten der kürzlich erschienen internationalen prospektiven longitudinalen Langzeitstudie des natürlichen Verlaufs (NHS) von 45 Patienten mit XLMTM (davon 11 deutsche Patienten) verglichen. Um das breite phänotypische Spektrum der Erkrankung hervorzuheben, präsentieren wir klinischen Verlauf von drei Patienten mit XLMTM.

Im Vergleich zeigt sich, dass die Patienten der deutschen Kohorte häufiger schwerer betroffen erscheinen. Eine weitere Erkenntnis ist eine deutliche diagnostische Zeitlücke zwischen den ersten klinischen Symptomen und der genetischen Diagnosebestätigung.

Dies zeigt, dass eine frühzeitige Wahrnehmung möglicher Symptome zu einer Betreuung in einem spezialisierten Zentrum führen sollte.

\section{P 37 Patientenrelevante Faktoren der gesundheitsbezogenen Lebensqualität und Versorgungssituation bei sporadischer Einschlusskörpermyositis - erster qualitativer Teil einer Krankheitskostenanalyse im Mixed-Methods Design}

Autoren Senn $K C^{1}$, Thiele $S^{2}$, Krause $S^{2}$, Schmidt $K^{3}$, Schmidt $J^{3}$, Walter $\mathrm{MC}^{2}$, Nagels $\mathrm{KH}^{1}$

Institutes 1 Universität Bayreuth, Lehrstuhl für Medizinmanagement und Versorgungsforschung, Bayreuth, Deutschland; 2 Friedrich-Baur-Institut, Ludwig-Maximilians-Universität, Neurologische Klinik und Poliklinik, München, Deutschland; 3 Universitätsmedizin Göttingen, Klinik für Neurologie,

Göttingen, Deutschland

DOI 10.1055/s-0041-1723712

Die sporadische Einschlusskörpermyositis (sIBM) ist die häufigste Form der Myositis jenseits des 50. Lebensjahres. Sie verläuft langsam-chronisch progredient und ist bisher nicht ursächlich therapierbar. Die empirische Evidenz zu gesundheitsbezogener Lebensqualität (HRQoL) und Versorgungssituation 
dieser Patienten ist unzureichend. Ziel der Studie ist die Vorbereitung empirischer Untersuchungen zur Versorgungssituation entlang der "patient journey" sowie die Identifikation der Einflussfaktoren auf die HRQoL von sIBM-Patienten. Diese qualitative Studie ist der erste Teil einer Krankheitskostenanalyse im sequenziellen Mixed-Methods Design. Eingeschlossen werden Patienten des deutschen IBM Patientenregisters (www.ibm-register.de) mit nach ENMC-Kriterien klinisch (probable) oder klinisch-pathologisch (certain) definierter sIBMDiagnose.

Für die im Alltagskontext interviewten Patienten $(n=7)$ sind neben den physischen Funktionseinschränkungen auch psychische und soziale Einflussfaktoren auf die HRQoL von hoher Bedeutung. Die Bewertung der HRQoL und Versorgung differenziert sich nach individueller Krankheitsprogression und Patientencharakteristika.

Untersuchung und Bewertungssystem leisten einen Beitrag zur patientenzentrierten Optimierung der gesundheitsökonomischen Methodik und eine erste qualitative Analyse der Versorgungssituation von sIBM-Patienten im Sinne der grundlagenorientierten Versorgungsforschung.

\section{P 38 Rapamycin als Therapieoption für Patienten mit Einschlusskörpermyositis - ein Fallbericht}

Autoren Pawlitzki $\mathrm{M}^{1}$, Labeit $\mathrm{B}^{2}$, Nelke $\mathrm{C}^{2}$, Rolfes $\mathrm{L}^{1}$, Ruck $\mathrm{T}^{2}$ Institutes 1 Klinik für Neurologie mit Institut für translationale Neurologie Münster, Klinik für Neurologie, Münster, Deutschland; 2 Klinik für Neurologie mit Institut für Translationale Neurologie Münster, Klinik für Neurologie, Münster, Deutschland DOI 10.1055/s-0041-1723927

Hintergrund Für die Einschlusskörpermyositis (IBM) existieren weiterhin keine zugelassenen Therapieoptionen. Insbesondere Therapien, die eine Stabilisierung oder Verbesserung der Motorik bewirken, fehlen. In einer kürzlich veröffentlichten Phase II Studie mit Rapamycin, einem mTOR-Inhibitor, ergaben sich Hinweise für eine Verlangsamung des Krankheitsprogresses über einen Therapiezeitraum von 12 Monaten, auch wenn der primäre Endpunkt verpasst wurde. Dies nahmen wir zum Anlass für einen off-label Therapieversuch bei einer schwer betroffenen IBM Patientin.

Fallbericht Eine 72-jährige Patientin mit bioptisch gesicherter IBM (Krankheitsdauer: 6 Jahre) stellte sich aufgrund einer zunehmenden Dysphagie vor. Nach der Diagnosestellung sei eine 4-jährige Therapie mit intravenösen Immunglobulinen erfolgt, jedoch ohne Krankheitsstabilisierung. Zum Zeitpunkt der Vorstellung bestand eine mittelschwere Tetraparese (MMT8 99/150), die Patientin war nur mit Gehstützen mobil. Wir leiteten daraufhin eine Therapie mit Rapamycin $2 \mathrm{mg} /$ Tag p.o. ein.

Ergebnisse Bereits nach 9 Monaten fiel eine deutliche klinische Verbesserung auf (MMT8 132/150). Nach einjähriger Therapie waren an der proximalen Muskulatur keine Paresen mehr eruierbar, die Patientin konnte ohne Unterstützung gehen. Hinsichtlich der Dysphagie zeigte sich in der funktionellen endoskopischen Schluck-Untersuchung ein stabiler Befund.

Schlussfolgerung Rapamycin scheint eine neue potenzielle Therapieoption für IBM Patienten zu sein.

\section{P 39 Auswirkungen eines Therapieabbruches mit Nusinersen bei SMA3}

Autoren Hiebeler $\mathrm{M}^{1}$, Stroth $\mathrm{I}^{1}$, Krause $\mathrm{S}^{1}$, Thiele $\mathrm{S}^{1}$, Reilich $\mathrm{P}^{1}$, Walter $\mathrm{MC}^{1}$ Institute 1 Friedrich-Baur-Institut, Neurologische Klinik und Poliklinik, Klinikum der Ludwig-Maximilians-Universität München, München, Deutschland

DOI 10.1055/s-0041-1723713

Die Spinale Muskelatrophie (SMA) ist eine progressive, autosomal-rezessive neurodegenerative Erkrankung mit einer Inzidenz von 1:10.000 Lebendgeburten. Mit besserem Verständnis der molekularen Basis der SMA in den letzten beiden Jahrzehnten richtete sich der Fokus therapeutischer Entwicklungen auf eine Erhöhung des Anteils an funktionsfähigem
SMN Protein, entweder durch Einschluss von Exon 7 in SMN2 Transkripte, Erhöhung der SMN2 Genexpression, oder durch direkten Genersatz von SMN1. Seit Juni 2017 steht mit Nusinersen/Spinraza ${ }^{\circledR}$ - ein Antisense Oligonukleotid, dass das pre-Messenger RNA Spleißen von Exon 7 des SMN2 Gens modifiziert, und so zu stabiler SMN Proteinexpression führt - erstmals eine wirksame krankheitsmodifizierende Therapie zur Verfügung. Wir berichten über den Therapieverlauf, den Therapieabbruch und die weitere Krankheitsentwicklung einer 46-jährigen Patientin mit SMA3.

Innovationen in der Wirtschaft

\section{P 40 Therapeutische Bewegungsgeräte bei der ALS - Anwendungshäufigkeit und subjektiver Nutzen in einer multizentrischen Beobachtungsstudie}

Autoren Gaudlitz $\mathrm{M}^{1}$, Spittel $\mathrm{S}^{2,1}$, Weyen $\mathrm{U}^{3}$, Grehl $\mathrm{T}^{4}$, Grosskreutz $\mathrm{J}^{5}$, Steinbach $R^{5}$, Weydt $P^{6}$, Günther $R^{7,8}$, Wolf $\rfloor^{9}$, Baum $P^{10}$, Metelmann $M^{10}$, Koch $J C^{11}$, Kettemann $D^{2}$, Norden $J^{2}$, Koc $Y^{2}$, Walter $B^{2}$, Münch $C^{2,1}$, Meier $\mathrm{A}^{2}$, Meyer $\mathrm{T}^{2,1}$

Institutes 1 Ambulanzpartner Soziotechnologie APST GmbH, Berlin, Deutschland; 2 Charité - Universitätsmedizin Berlin, Klinik für Neurologie, Ambulanz für ALS, SMA und andere Motoneuronerkrankungen, Berlin, Deutschland; 3 Berufsgenossenschaftliches Universitätsklinikum Bergmannsheil, Klinik für Neurologie, Ambulanz für ALS und andere Motoneuronerkrankungen, Bochum, Deutschland; 4 Alfried Krupp Krankenhaus, Klinik für Neurologie, Ambulanz für ALS und andere Motoneuronerkrankungen, Essen, Deutschland; 5 Universitätsklinikum Jena, Klinik für Neurologie, Neuromuskuläre und Motoneuron Ambulanz, Jena, Deutschland; 6 Universitätsklinikum Bonn, Klinik für Neurodegenerative Erkrankungen, Bonn, Deutschland; 7 Universitätsklinikum Carl Gustav Carus, Klinik für Neurologie, Dresden, Deutschland; 8 Deutsches Zentrum für Neurodegenerative Erkrankungen, Standort Dresden, Dresden, Deutschland; 9 Diakonissenkrankenhaus Mannheim, Klinik für Neurologie, Mannheim, Deutschland; 10 Universitätsklinikum Leipzig, Klinik und Poliklinik für Neurologie, ALS-Ambulanz, Leipzig, Deutschland; 11 Universitätsmedizin Göttingen, Klinik für Neurologie, Göttingen, Deutschland DOI 10.1055/s-0041-1723714

Hintergrund Therapeutische Bewegungsgeräte (TB) ermöglichen eine gerätegestützte Physiotherapie (GPT) in der Häuslichkeit. Die Beobachtungsstudie erfasst die TB-Anwendungshäufigkeit, das subjektive Erleben der GPT und die Weiterempfehlung des TB.

Methode Realisiert wurde eine prospektive Kohortenstudie an 9 ALS-Zentren (02/2019-10/2020). Einschlusskriterien: ALS, Extremitätenparesen, TB-Versorgung, Teilnahme bei "Ambulanzpartner". Die Anwendungshäufigkeit und der subjektive Nutzen der GPT wurden durch eine Numerische Ratingskala (0-10 Punkte) erfasst. Die Weiterempfehlungswahrscheinlichkeit des TB wurde mit dem Net Promotor Score (NPS) ermittelt.

Ergebnisse 106 Patienten wurden analysiert. Folgende wöchentliche Anwendungen wurden identifiziert: $1-2=20 \% ; 3-4=20 \% ; 5-7=44 \% ;>7=16 \%$. Folgende Behandlungsbereiche erreichten eine besondere Zustimmung: Verbesserung des allgemeinen Wohlbefindens (98\%) und des Gefühls "etwas geschafft zu haben" sowie Verminderung des Gefühls des "Einrostens" und der "Bewegungslosigkeit" (97\%). Der NPS für das TB lag bei +61.

Schlussfolgerungen Die Mehrheit der ALS-Patienten (61\%) nutzt den TB mindestens 5 Mal pro Woche und erhielt damit eine hochfrequente GPT in Ergänzung zur PT. Die individuellen Behandlungsziele einer GPT wurden überwiegend erreicht. Die Zufriedenheit mit dem TB ist außergewöhnlich hoch (ein NPS > 50 gilt als "exzellent"). Die Ergebnisse unterstützen die weitere Etablierung der GPT im Versorgungskonzept der ALS. 
P 41 Safety, $\beta$-sarcoglycan Expression and Functional Outcomes from Systemic Gene Transfer of rAAVrh74. MHCK7.SGCB in Patients with Limb-Girdle Muscular Dystrophy Type 2E (LGMD2E)

\author{
Authors Rodino-Klapac $\mathrm{L}^{1}$, Pozsgai $\mathrm{E}^{1,2}$, Lewis $\mathrm{S}^{1,2}$, Griffin $\mathrm{D}^{1,2}$, \\ Meadows $\mathrm{A}^{1,2}$, Lehman $\mathrm{K}^{2}$, Church $\mathrm{K}^{2}$, Miller $\mathrm{N}^{2}$, lammarino $\mathrm{M}^{2}$, Lowes $\mathrm{L}^{2}$, \\ Mueller-York $A^{1}$, Mendell j j $^{2,3}$ \\ Institute 1 Sarepta Therapeutics, Inc., Cambridge, Vereinigte \\ Staaten; 2 Center for Gene Therapy, The Research Institute at Nationwide \\ Children's Hospital, Columbus, $\mathrm{OH}$, Vereinigte Staaten; 3 The Ohio State \\ University, Department of Pediatrics and Neurology, Columbus, $\mathrm{OH}$, Vereinigte \\ Staaten
}

DOI 10.1055/s-0041-1723715

Introduction LGMD2E is progressive and debilitating.

Methods In this first-in-human, single-center, open-label, systemic gene delivery, Phase 1/2 study, 6 patients with LGMD2E received self-complementary rAAVrh74.MHCK7.hSGCB in escalating doses. Patients: 4-15y, SGCB gene mutation (both alleles), no rAAVrh74 antibodies,> $40 \%$ on 100-meter timed test. Cohort $1(n=3)$ and Cohort $2(n=3)$ received single IV infusion of $5 \times 10^{13}$ $\mathrm{vg} / \mathrm{kg}$ rAAVrh74.MHCK7.hSGCB and $2 \times 10^{14} \mathrm{vg} / \mathrm{kg}$, respectively. Prednisone 1 $\mathrm{mg} / \mathrm{kg} /$ day initiated $1 \mathrm{~d}$ before (tapering after 30-60 days). Endpoints: Primary - safety. Secondary - SGCB expression from baseline to week 8. Other - CK decrease; functional endpoints (North Star Assessment of Limb-girdle Muscular Dystrophies [NSAD] and timed functional tests [100-meter walk/run, 10-meter walk/run, 4-stair climb, and time to rise]).

Results Systemic administration of rAAVrh74.MHCK7.hSGCB is well-tolerated; no unexpected immunological responses observed. Efficient transduction, robust SGCB protein expression seen in all post-infusion, leading to re-constitution of the sarcoglycan complex and reductions in CK. 6 months post-infusion, patients in both cohorts had improvement in functional measures compared with baseline. 18-month follow-up data in Cohort 1 demonstrate improvements in NSAD and timed tests maintained over baseline.

Discussion These data suggest long-term efficacy of rAAVrh74.MHCK7.hSGCB gene transfer, supporting advancement of the clinical development program.

\section{P 42 Long-term Safety and Efficacy of Exon 53 Skipping in Male Patients With Duchenne Muscular Dystrophy}

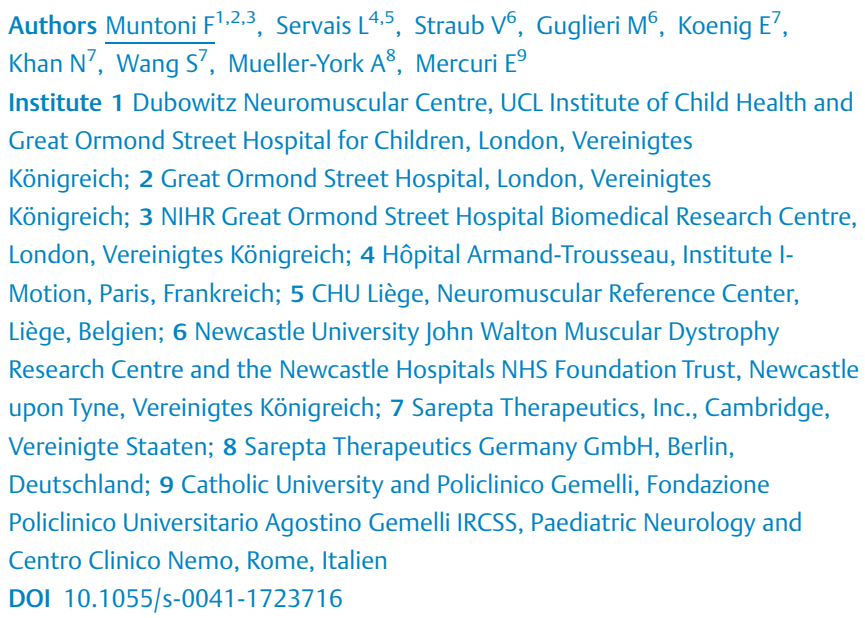

Results Median age was 8 years at recruitment. Median golodirsen exposure was 168 weeks and safety data were collected up to 189 weeks. All patients experienced $\geq 1$ treatment-emergent adverse event $(A E)$, including rhinitis, nasopharyngitis, headache, and proteinuria. Overall, 12 patients (48\%) had mild, 8 (32\%) had moderate, and 5 (20\%) had severe AEs. All severe AEs were nonserious and considered unrelated to golodirsen. There were no deaths and no discontinuations due to an AE. At Week 48, golodirsen increased exon 53 skipping (29-fold) and dystrophin protein (16-fold). Muscle and pulmonary function were evaluated over 144 weeks and compared with matched natural history cohorts (to be presented).

Discussion Long-term treatment with golodirsen demonstrated acceptable safety/tolerability. These data and dystrophin expression support golodirsen as a treatment option in patients with DMD amenable to exon 53 skipping.

\section{P 44 Stationäre Aufwendungen für XLMTM Patienten - eine Erhebung in deutschen Gesundheitseinrichtungen}

Autoren Schmitt $\mathrm{D}^{1}$, Slocomb $\mathrm{T}^{1}$, Hathway $\mathrm{J}^{2}$, Jensen $\mathrm{I}^{2}$ Institutes 1 Audentes Therapeutics, San Francisco, CA, Vereinigte Staaten; 2 Precision Health Economics, Boston, MA, Vereinigte Staaten DOI 10.1055/s-0041-1723717

Ziele Die X-chrom. myotubuläre Myopathie (XLMTM) ist eine seltene angeborene Myopathie. Wir untersuchten die Anzahl der stationären Aufnahmen, die Verweildauer (VWD) und die Kosten in Krankenhäusern für Akutversorgung (AV) und Langzeitbeatmung (LZB).

Methoden Es wurde eine Querschnitts-Stichprobe von 5 XLMTM-Patienten ausgewertet. Das Alter bei Aufnahme von zwei der drei LZB-Patienten betrug > 3,5 Jahre und war für den dritten unbekannt. Beide AV-Patienten waren < 1 Jahr alt bei Erstaufnahme. Gesamt-VWD, Häufigkeit der Einweisungen und Krankenhauskosten wurden bewertet. Die Kosten für Patienten in AV-Krankenhäuser basierten auf DRG's, die Kosten für LZB-Patienten beruhten auf Tagessätzen.

Ergebnisse Bei einem LZB-Patient entstanden 71\% der gesamten VWD (8.373 Tage) und $67 \%$ der Gesamtkosten (5.072.662 €). Die LZB-Patienten machten $97 \%$ der Patiententage und $90 \%$ der Gesamtkosten aus. Der durchschnittliche LZB-Tagessatz betrug 579,63 €. Bei AV-Patienten entstanden Krankenhauskosten in Höhe von $127.847 €$ und $376.894 €$ mit einem Durchschnitt von 1.832 $€ /$ Tag. Bei einem AV-Patienten wurden 6 Aufnahmen über 227 Tage mit einer kumulierten VWD von 111 Tagen und bei dem zweiten Patienten wurden 29 Aufnahmen über 2.042 Tage und eine kumulative VWD von 150 Tagen erhoben.

Schlussfolgerung Neben den in Vordergrund stehenden Belastungen sind in der ökonomischen Betrachtung die Verweildauer in LZB, die Anzahl der Aufenthalte in $\mathrm{AV}$ und die kumulativen Kosten die wesentlichen Aufwendungen.

Methode clinico-genet-omique

\section{P 45 Die Pathogenität einer häufigen CAPN3 c.1746- 20C> G Spleißvariante}

Autoren Mroczek $\mathrm{M}^{1}$, Hudson $\mathrm{J}^{2}$, Barresi $\mathrm{R}^{1}$, Inashkina $\mathrm{I}^{3}$, Lace $\mathrm{B}^{3}$, Stavusis $\mathrm{J}^{3}$, Töpf $A^{1}$, Duff $]^{1}$, Sobalska-Kwapis $M^{4}$, Strapagiel $D^{4}$, England $E^{5}$, Chao $K^{5}$, MacArthur $\mathrm{D}^{5}$, Straub $\mathrm{V}^{1}$

Institutes 1 Newcastle University, Translational and Clinical Research Institute, Newcastle upon Tyne, Vereinigtes Königreich; 2 NHS, Highly Specialized Service (HSS) for LGMDs, Newcastle upon Tyne, Vereinigtes Königreich; 3 Latvian Biomedical Research and Study Centre, Riga, Lettland; 4 The Biobank Lab, Department of Molecular Biophysics, Lodz, Polen; 5 Massachusetts General Hospital, Analytic and Translational Genetics Unit, Boston, MA, Vereinigte Staaten

DOI $10.1055 / \mathrm{s}-0041-1723718$ 
Fragestellung Die Gliedergürteldystrophie LGMD2A (LGMD 1R Calpain-3-related) ist die häufigste Form der LGMD. Die CAPN3 c.1746-20C> G-Variante hat eine Populationshäufigkeit von über> $1 \%$ und es bestehen widersprüchliche Informationen bzgl. ihrer Pathogenität.Ziel unserer Arbeit ist die Pathogenität dieser Variante zu definieren.

Methoden Pathogenitätsstudien wurden durch Analyse von LGMD-Kohorten, Populationsanalysen und Funktionsstudien (Spleißanalysen, Western Blot) durchgeführt.

Ergebnisse LGMD Kohorten: In mehr als 2.000 Patienten mit LGMD identifizierten wir die Variante c.1746-20C> G 26-mal in Heterozygotie und 1-mal in Homozygotie. Die Variante trat zusammen mit CAPN3 c.598_612del, c.550delA, p.Arg39Cys, p.Gly234Arg und anderen pathogenen heterozygoten CAPN3-Varianten auf. Die meisten Patienten waren polnischer oder osteuropäischer Herkunft. In all diesen Fällen war die Calpain3-Expression im Western Blot reduziert, und die Muskel-MRT und Phänotypen konnten mit LGMD2A vereinbart werden.

Populationsstudien: Populationsstudien legen eine Allelfrequenz von 0,017 in Polen, 0,015 in Estland und 0,018 in Lettland nahe. Ein homozygotes gesundes Individuum wurde einmal in unseren Populationsstudien identifiziert und laut gnomAD gibt es 3 weitere gesunde Homozygoten (von 282.874).

Schlussfolgerungen Daten deuten auf eine hypomorphe Variante hin. Seltene Krankheiten können durch relativ häufige Varianten nach einem quasi-Mendelschen Vererbungsmodell geerbt werden.

\section{$\mathrm{P} 46 \mathrm{Bi}$-allelic truncating mutations in VWA1 cause neuromyopathy}

Authors Deschauer $\mathrm{M}^{1}$, Hengel $\mathrm{H}^{2}$, Rupprich $\mathrm{K}^{3}$, Kreiß $\mathrm{M}^{4}$, SchlotterWeigel $\mathrm{B}^{5}$, Grimmel $\mathrm{M}^{6}$, Admard $\mathrm{J}^{6}$, Schneider $\mathrm{I}^{7}$, Alhaddad $\mathrm{B}^{8}$, Gazou $\mathrm{A}^{6}$, Sturm $\mathrm{M}^{6}$, Vorgerd $\mathrm{M}^{9}$, Balousha $\mathrm{G}^{10}$, Balousha $\mathrm{O}^{11}$, Falna $\mathrm{M}^{11}$, Kirschke $\rfloor^{12}$, Kornblum $C^{13}$, Jordan $B^{7,14}$, Kraya $T^{7}$, Strom $T^{8}$, Weis $\rfloor^{15}$, Schöls $L^{2}$, ScharaSchmidt $\mathrm{U}^{3}$, Zierz $\mathrm{S}^{7}$, Riess $\mathrm{O}^{6}$, Meitinger $\mathrm{T}^{8}$, Haack $\mathrm{T}^{6}$

Institute 1 Klinikum rechts der Isar der Technischen Universität München, Klinik und Poliklinik für Neurologie, München, Deutschland; 2 University of Tübingen, Department of Neurology and Hertie-Institute for Clinical Brain Research, Tübingen, Deutschland; 3 University Hospital Essen, Department of Neuropediatrics, Essen, Deutschland; 4 University of Bonn, Institute of Human Genetics, Bonn, Deutschland; 5 Ludwig Maximilian University Munich, Department of Neurology, München, Deutschland; 6 University of Tübingen, Institute of Medical Genetics and Applied Genomics, Tübingen,

Deutschland; 7 University of Halle-Wittenberg, Department of Neurology, Halle a. d. S., Deutschland; 8 Institute of Human Genetics, Technical University of Munich, München, Deutschland; 9 Department of Neurology, University Hospital Bergmannsheil, Bochum, Deutschland; 10 Al-Quds University, Department of Pathology and Histology, Jerusalem, Palästinensische Autonomiegebiete; 11 Al-Quds University, Faculty of Medicine, Jerusalem, Palästinensische Autonomiegebiete; 12 Technical University of Munich, Department of Diagnostic and Interventional Neuroradiology, München, Deutschland; 13 University Hospital Bonn, Department of Neurology, Bonn, Deutschland; 14 University Hospital Heidelberg, Department of Neurology, Heidelberg, Deutschland; 15 RWTH Aachen University, Institute for Neuropathology, Aachen, Deutschland

DOI $10.1055 / \mathrm{s}-0041-1723719$

The von Willebrand Factor A Domain-Containing Protein 1, encoded by the VWA1 gene, is an extracellular matrix protein expressed in muscle and peripheral nerve. It interacts with collagen VI and perlecan, two proteins that are affected in hereditary neuromuscular disorders. WWA1 is known to compromise peripheral nerves in a WWA1 knock-out mouse model. Exome sequencing led us to identify bi-allelic loss of function variants in VWA1 as the molecular cause underlying a so far genetically undefined neuromuscular disorder. We detected six different truncating variants in 15 affected individuals from six families of German, Arabic, and Roma descent. Disease manifested in childhood or adulthood with proximal and distal muscle weakness predominantly of the lower limbs. Myopathological and neurophysiological findings were indicative of combined neurogenic and myopathic pathology. Early childhood foot deformity was frequent, but no sensory signs were observed. Our findings establish VWA1 as a new disease gene confidently implicated in this autosomal recessive neuromyopathic condition presenting with child-/adult-onset muscle weakness as a key clinical feature.

\section{P 47 Splice-Regulation durch CHERP in Myotoner Dystrophie Typ 1}

Authors Todorow $\mathrm{V}^{1}$, Kao $S Y^{2}$, Hintze $\mathrm{S}^{1}$, Meinke $\mathrm{P}^{1}$, Schoser $\mathrm{B}^{1}$, Spletter $\mathrm{M}^{2}$ Institute 1 FRIEDRICH-BAUR-INSTITUT/Klinikum der Universität München,

München, Deutschland; 2 Biomedizinisches Zentrum LMU, München,

Deutschland

DOI 10.1055/s-0041-1723720

Einleitung Myotone Dystrophie Typ 1 (DM1) wird durch eine Repeat-Expansion im DMPK Gen verursacht. Klinisch sind Myotonie und Muskeldystrophie die am stärksten ausgeprägten Symptome. Der bisher beschriebene Pathomechanismus ist die Repeat-Längen-abhängige Ausbildung sekundärer RNA-Strukturen, welche zu einer verstärkten Interaktion mit den Splice-Faktoren MBNL und CELF führen. Allerdings ist unklar ob der gesamte multisystemische Phänotyp dadurch alleine erklärt werden kann. Um den DM1 Pathomechanismus weiter zu untersuchen haben wir einen weiteren Splice-Faktor untersucht: CHERP. Drosophila-Mutanten des CHERP-homologen Gens (Scaf6) haben Defekte in der Muskelentwicklung und -funktion, was zur Flugunfähigkeit der Fliegen führt. Die Expression von CHERP ist in DM1 Myoblasten und Myotuben verändert.

Material/Methode In C2C12-Myoblasten sowie in primären humanen Myoblasten wurden mittels IP-MS Interaktionspartner identifiziert. Weiterhin wurde in C2C12-Myoblasten ein Knockdown von CHERP durchgeführt um regulierte Gene mittels RNAseq zu identifizieren.

Ergebnisse CHERP scheint mit dem SF3b Subkomplex für 3" Splicesite-Erkennung zu interagieren und reguliert die Expression von Genen, die zu muskelspezifischen Funktionen sowie Zellzyklus-Kontrolle assoziiert sind.

Diskussion Die Gene, welche durch CHERP reguliert werden, und die Expressionsveränderung von CHERP in DM1 legen eine funktionelle Beteiligung dieses Proteins an der DM1 Pathologie nahe.

\section{P 48 Charakterisierung neuer humanisierter Dysferlinopathie Mausmodelle}

Autoren Zhogov $A^{1}$, Fernandez $\mathrm{HE}^{1}$, Kühn $\mathrm{R}^{2}$, Spuler $\mathrm{S}^{1}$

Institutes 1 Experimental and Clinical Research Center, a joint cooperation of Charité Universitätsmedizin Berlin and Max-Delbrück Center for Molecular Medicine, Muscle Research Unit, Berlin, Deutschland; 2 Max-Delbrück Center for Molecular Medicine, Berlin, Deutschland

DOI 10.1055/s-0041-1723721

Fragestellung Dysferlinopathie ist eine autosomal-rezessiv vererbte Muskeldystrophie, die von einem Mangel an Dysferlin Protein aufgrund von Mutationen im DYSF-Gen verursacht wird. Eine Therapie gibt es derzeit nicht. Eine Korrektur der Mutation direkt in Muskeln der Patienten könnte durch CRISPR/Cas9 basierende Systeme gelingen. Um diese Möglichkeit präklinisch zu testen, ist die Entwicklung geeigneter Mausmodelle notwendig.

Methoden Mit Hilfe von CRISPR/Cas wurden zwei Mausmodele generiert. Bei HumEx44_WT wurde Dysf Exon 44 der Maus mit einem gesunden menschlichen DYSF Exon 44 ersetzt. HumEx44_MUT tragen den menschlichen DYSF Exon 44 mit der Gründermutation c.4872_4876delinsCccC. Skelettmuskelgewebe wurde histologisch analysiert. Muskelfasertyp, Geschlechts und Alter spezifische Quantifizierung der Veränderungen im Vergleich zu C57BL/6N Mäusen steht im Vordergrund.

Ergebnisse HumEx44_WT Mäuse exprimieren Dysferlin und zeigen keinen pathologischen Phänotyp. HumEx44_MUT Mäuse exprimieren kein Dysferlin und zeigen 
schon im Alter von 8 Wochen dystrophische Veränderungen. Mit Alter zeigt sich eine deutliche Progredienz. Männliches Geschlecht ist deutlich schwerer betroffen und es gibt Hinweise auf Unterschiede zwischen Muskelfasertypen.

Schlussfolgerung Unsere Ergebnisse werfen neues Licht auf die Unterschiede zwischen Geschlecht und Fasertyp bei Dysferlinopathie und stellen ein wichtiges Modell vor, um in vivo gene editing Ansätze für eine DYSF Gründermutation zu testen.

\section{P 49 Novel form of congenital myopathy caused by bi-allelic mutations in uncoordinated mutant number- 45 myosin chaperone}

Authors Cirak $\mathrm{S}^{1,2}$, Dafsari $\mathrm{HS}^{1,2}$, Kocatürk NM ${ }^{1,2}$, Diofano $\mathrm{F}^{3}$, Daimagüler $\mathrm{H}^{1,2}$, Dötsch $\mathrm{J}^{2}$, Brunn $\mathrm{A}^{4}$, Weis $\mathrm{J}^{5}$, Deckert $\mathrm{M}^{4}$, Just $\mathrm{S}^{6}$ Institute 1 Zentrum für Molekulare Medizin, Kinder-und Jugendheilkunde, Köln, Deutschland; 2 Universitätsklinikum Köln, Kinder-und Jugendheilkunde, Köln, Deutschland; 3 Universitätsklinikum Ulm, Ulm,

Deutschland; 4 Universitätsklinikum Köln, Neuropathologie, Köln, Deutschland; 5 Universitätsklinikum Aachen, Neuropathologie, Aachen, Deutschland; 6 Universitätsklinikum Ulm, Molecular Cardiology, Ulm, Deutschland

DOI 10.1055/s-0041-1723722

Congenital myopathies (CM) form a genetically heterogeneous group of disorders, only $60 \%$ can be genetically solved.

We recruited an 11year old male of consanguineous parents with proximal weakness, Gower"s sign, without cardiomyopathy. We performed exome sequencing and data analysis was done with Varbank2 according to an autosomal recessive inheritance. We investigated the effect of the missense mutation by complementation assay on the zebrafish steif mutant, an unc-45b loss-offunction model.

We have discovered and published a novel genetically defined form of CM due to a novel homozygous missense mutation in UNC45B(NM_173167.2: c.2261G > A, p.R754Q)(Dafsari 2019). Muscle biopsy showed core-like structures in the center of muscle fibers in NADH histochemistry. Electron microscopy showed numerous focal core-like alterations of myofibrillar architecture with Zbands streaming.

Three isoforms ofUNC45B are highly expressed in skeletal muscle, only one in cardiac muscle. Due to its high evolutionary conservation, a loss of UNC45 results in different pathological conditions in various species: a knockdown of unc-45 resulted in dilated cardiomyopathy and a reduced muscle contractility in D. melanogaster. In unc-45b knockdown zebrafish and also in steif mutants, disrupted myofibrillogenesis associated cardiac dysfunction and paralysis was observed. Injection of mutant unc-45bmRNA did rescue the steif mutant in contrast to wt mRNA confirming the pathogenicity of the missense mutation.

\section{P 50 Novel bi-allelic variants in KIF21Acause a novel phenotype of fetal akinesia with neurodevelopmental defects}

Autoren Cirak $\mathrm{S}^{1,2}$, Özdemir Ö1,2,3 , Daimagüler $\mathrm{H}^{1,2}$, Karaca $\mathrm{E}^{4}$, Sprute $\mathrm{R}^{1,2}$ Pergande $\mathrm{M}^{1,2}$, Punetha $\mathrm{J}^{5}$, Bhola $P T^{6}$, Kostera-Pruszczyk $A^{7}$, Nürnberg $\mathrm{P}^{8}$, Posey $\mathrm{JE}^{5}$, Rauch $\mathrm{A}^{9}$, Kernohan $\mathrm{KD}^{6}$, Shukla $A^{10}$, Lupski JR $\mathrm{R}^{5}$

Institutes 1 Zentrum für Molekulare Medizin, Kinder-und Jugendheilkunde,

Köln, Deutschland; 2 Universitätsklinikum Köln, Kinder-und Jugendheilkunde,

Köln, Deutschland; 3 Acıbadem Mehmet Ali Aydınlar University, Istanbul,

Türkei; 4 Izmir Biomedicine and Genome Center, Izmir, Türkei; 5 Baylor College

of Medicine, Houston, TX, Vereinigte Staaten; 6 University of Ottawa, Ottawa,

Kanada; 7 Medical University of Warsaw, Warschau, Polen; 8 Cologne Center

for Genomics, Köln, Deutschland; 9 University of Zurich, Schlieren,

Schweiz; 10 Kasturba Medical College, Manipal, Indien

DOI 10.1055/s-0041-1723723
Fetal akinesia (FA) is a complex disease entity, sharing arthrogryposis as a common feature. We report 5 unrelated individuals with biallelic variants in the kinesin family member 21A gene (KIF21A), a member of the kinesin- 4 family that is functional in axon growth and guidance by transporting cargo anterograde to the synapses. Using whole-exome-sequencing we identified 8 mutations that are loss of function mutations predicted to cause premature stop codon or essential splice site mutations that would lead to aberrant KIF21Aisoforms. We showed that the affected individuals with those variants had severe phenotypes with neurodevelopmental disorder, structural brain abnormalities, arthrogryposis of multiple joints, muscular hypotonia, and hypokinesia. Furthermore, all these phenotypes strongly correlate with the recently published porcine phenotype that also has bi-allelic truncating variants, with a few of patients showing features of a neuropathy. In contrast with our findings, previous reports have indicated that the known phenotypic effect of KIF21A caused by the heterozygous variants that lead to missense mutations or deletions and are significantly clustered in the motor domain or coiled-coil region of KIF21A, which in turn causes an isolated ophthalmologic phenotype with congenital fibrosis of the extraocular muscles type 1 (CFEOM1). Conclusively, we describe a novel disease in the FA spectrum with a primary neurogenic defect due to bi-allelic variants in the KIF21A.

\section{P 51 Combined biochemical and targeted-next generation sequencing panel for differential diagnosis of inherited myopathies}

Authors Wiesinger $\mathrm{T}^{1}$, Kasper $\mathrm{D}^{1}$, Schwarz $\mathrm{M}^{1}$, Mechtler $\mathrm{T}^{1}$, LiebmannReidl $S^{1}$, Streubel B

Institute 1 Archimedlife Science GmBH, R\&D, Wien, Österreich; 2 Medical University of Vienna, Department of Pathology, Wien, Österreich

DOI 10.1055/s-0041-1723724

Myopathies are neuromuscular disorders represented by muscle weakness, muscle cramps, stiffness and spasms. Myopathies are occasionally evident at birth and can be inherited (e.g. muscular dystrophies) or acquired (e.g. muscle cramps). In general, they can be categorized into different groups: (1) congenital myopathies, (2) muscular dystrophies and (3) glycogen storage diseases (e.g. Pompe disease- impaired enzymatic degradation of glycogen).

Our medical laboratory has developed and validated a novel approach in combining a biochemical and targeted-NGS muscle panel for differential diagnosis of myopathies using Dried Blood Spots. After an initial pre-test for alphaglucosidase (for Pompe disease) using tandem mass spectrometry as well as gene copy determination of SMN1 (for 5q-SMA), a targeted-NGS panel is performed depending on CK level and clinical symptoms. Depending on the age (children vs adults), different gene panels were developed up to 30 genes in case of children and 17 genes for adults together with clinical specialists.

Within 8 months already one Pompe and one SMA patient as well as six individuals with different mutations (e.g. RYR1- central core myopathy, COL6A3 congenital muscle dystrophy) were identified. This targeted work-flow does not cover a whole exom screening but highlights the benefits to determine the most common variants based on clinical symptoms, age and CK levels for a first fast and clinical-based differential diagnosis.

\section{P 52 Metabolisches Profiling primärer Muskelzellkulturen erblicher Muskelerkrankungen}

Autoren Meinke $\mathrm{P}^{1}$, Hintze $\mathrm{S}^{1}$, Schoser $\mathrm{B}^{1}$

Institute 1 Friedrich-Baur-Institut, München, Deutschland

DOI 10.1055/s-0041-1723725

Einleitung Der Energiestoffwechsel ist eine grundlegende Funktion jeder Zelle. Es gibt erbliche metabolische Myopathien mit direkter Störung des Energiestoffwechsels (z.B. mitochondriale Erkrankungen, Glykogenspeichererkrankungen, Lipidmyopathien). Zusätzlich sind sekundäre metabolische Veränderungen bei einigen Erkrankungen beschrieben (u.a. Myotone Dystrophien, FSHD). Ein energetisches Profiling der metabolischen Aktivität 
primärer Muskelzellkulturen kann die pathologische Relevanz bei einzelnen Erkrankungen weiter aufklären. Dies kann genutzt werden um neue pathophysiologische Aspekte einzelner Myopathien zu untersuchen, und evtl. neue therapeutische Ansätze zu erarbeiten.

Material/Methode Mittels des SeaHorse XFp Analyzers wurden in vitalen humanen Muskelzellkulturen glykolytische und mitochondriale Aktivität sowie die Abhängigkeit von Energieträgern gemessen.

Ergebnisse Wir konnten bisher nicht beschriebene neue metabolische Profile für einzelne Erkrankungen nachweisen.

Diskussion Unsere Untersuchungen ergeben erste Hinweise auf bisher nicht beschrieben pathophysiologische Zusammenhänge. Das ermöglicht weitere Untersuchung mit dem Ziel diese spezifischen pathophysiologischen Aspekte zu therapieren.

\section{P 53 Detection of a pericentric inversion in DMD by Whole Genome Sequencing (WGS)}

Authors Zaum AK ${ }^{1}$, Nanda I ${ }^{1}$, Kress $W^{1}$, Rost $S^{1}$

Institute 1 University of Würzburg, Department of Human Genetics,

Würzburg, Deutschland

DOI 10.1055/s-0041-1723726

Introduction The dystrophinopathies (Duchenne (DMD) and Becker muscular dystrophy (BMD)) are caused by mutations in the X-linked dystrophin gene $(D M D)$. Standard diagnostics consists of multiplex ligation-dependent probe amplification (MLPA) and sequencing of all coding exons, due to the most common types of variants.

Methods We present the case of a boy with clinical signs of DMD. His brother was also suffering from muscular dystrophy. Standard molecular diagnostics yielded no pathogenic variant for index or brother. WGS of the index patient was performed but only the sequence of the complete DMD gene was analysed.

Results The coverage data of the DMD gene revealed a large deletion in the intron 44. At the borders of the deletion, the alignment data showed hybridreads, consisting of partial DMD sequence and an unknown sequence. The unknown sequences could be mapped to a genomic position at Xq13.3. The orientation of the sequences led to the hypothesis of an inversion. Sanger sequencing of the breakpoints and chromosome analysis confirmed the inversion.

Discussion We present the case of a boy suffering from DMD due to a pericentric inversion of the $\mathrm{X}$ chromosome including DMD. Pericentric inversions are a very rare cause of DMD and only few cases have been reported so far. We could show that WGS is a useful tool to solve these cases. It should be considered in the future to diagnose unsolved cases.

\section{P 54 Automatisierte Segmentierung durch maschinelles Lernen von epidermalen Nervenfasern (Small Fiber) in Hautbiopsien}

Authors Zunk $L^{1}$, Sehring $J^{1}$, Bajors $L^{1}$, Amsel $D^{1}$, Schänzer $A^{1}$ Institute 1 Institut für Neuropathologie Gießen, Neuropathologie, Gießen, Deutschland DOI 10.1055/s-0041-1723727

Einleitung Der zunehmende Forschungsbedarf im Bereich neuropathischer Veränderungen der kleinen Nervenfasern (Small Fiber) der Haut erfordert eine Steigerung der Effektivität der Analyseverfahren. Durch den Einsatz automatischer Verfahren zur Detektion der Nervenfasern und Analyse zusätzlicher morphologischer Parameter kann die manuelle Diagnostik erweitert werden.

Material/Methode Hautbiospien von 24 erkrankten Patienten und 7 gesunden Probanden wurden nach standardisierten Verfahren aufgearbeitet. An Dickschnitten wurde eine Immunfluoreszenzfärbung mit Antikörper gegen PGP9.5 zur Darstellung der intraepidermalen Nervenfasern durchgeführt. Die Kernfärbung erfolgte mit DAPI. Die Schnitte wurden mit einem AxioScan.Z1 digitalisiert. Mithilfe von Methoden der Bildverarbeitung und des Maschinellen Lernens wird eine Segmentierung der Epidermis und Nervenfasern erzielt.

Ergebnisse Die Genauigkeit des Verfahrens zur Detektion und Segmentierung von Nervenfasern und Epidermis wir durch den Vergleich mit einer manuellen Annotation evaluiert. Die Annotation wird durch den Abgleich mit der Auszählung am Mikroskop validiert.

Diskussion Die Studie evaluiert in wie weit ein unabhängigeres objektiveres Verfahren dafür geeignet ist, die manuelle Auszählung zu unterstützen. Durch den Einsatz eines automatisierten Verfahrens weitere morphometrische Parameter, wie Nervenfaserlänge oder -Abstand, zu erfassen. Dies könnte eine Grundlage für zukünftige Studien darstellen.

\section{Differentialdiagnostik}

\section{P 55 Camptocormie bei M. Parkinson - diagnostische und therapeutische Implikationen}

Autoren Rosenbohm $A^{1}$, Wassner $A^{1}$, Ludolph AC $^{1}$, Kassubek J

Institute 1 Universität Ulm, Neurologie, Ulm, Deutschland DOI 10.1055/s-0041-1723728

Einleitung Es ist weiterhin nicht geklärt, welcher pathophysiologische Zusammenhang bei M. Parkinson zu einer Camptocormie mit paravertebraler Muskelschwäche führt. Viele Patienten entwickeln Paresen der paravertebralen Muskulatur, die nicht auf L-DOPA ansprechen, wohingegen in anderen Fällen eine paravertebrale Dystonie vermutet wird.

Methoden Wir berichten über eine Kohorte von 29 Parkinson Patienten, die eine bioptisch untersuchte Camptocormie aufweisen und erörtern die diagnostischen und therapeutischen Optionen.

Ergebnisse 21/29 Patienten zeigten in der Muskelbiopsie der paravertebralen Muskulatur neben myopathischen Veränderungen eine Myositis, am ehesten einer Polymyositis entsprechend. 17/21 Patienten mit myositischen Veränderungen erhielten eine Immunsuppression. 10/17 Patienten unter Prednisolon bzw. Azathioprin reagierten mit einer partiellen oder vollständigen Verbesserung der Paresen bis hin zur Vollremission.

Diskussion Histologische Veränderungen bei M. Parkinson mit Camptocormie zeigen kein einheitliches Bild. Neben meist myopathischen Veränderungen zeigten sich auch lokale Myositiden, die in $>50 \%$ erfolgreich auf eine immunsuppressive Therapie ansprachen. Dies ist mit der postulierten Überbeanspruchung der Rückenmuskulatur bei M. Parkinson nicht vereinbar, sondern stellt vielmehr eine Diagnose mit spezifischen therapeutischen Optionen dar. Wir empfehlen bei M. Parkinson mit Camptocormie unbedingt eine histologische Diagnosesicherung.

\section{P 56 Muskelatrophie, Faszikulationen und Gewichtsabnahme: immer ein Red-Flag für eine Motoneuronerkrankung?}

Autoren Schöne $U^{1}$, Brunkhorst $\mathrm{R}^{1}$, Gezer $\mathrm{D}^{2}$, Nikolin $\mathrm{S}^{3}$, Weis $\mathrm{J}^{3}$, Schulz JB ${ }^{1}$, Dohrn $\mathrm{MF}^{1,4}$

Institutes 1 Uniklinik RWTH Aachen, Klinik für Neurologie, Aachen, Deutschland; 2 Uniklinik RWTH Aachen, Klinik für Hämatologie und Onkologie, Aachen, Deutschland; 3 Uniklinik RWTH Aachen, Institut für Neuropathologie, Aachen, Deutschland; 4 University of Miami, Miller School of Medicine, Dr. John T. Macdonald Foundation, Department of Human Genetics and John P. Hussman Institute for Human Genomics, Miami, FL, Vereinigte Staaten DOI 10.1055/s-0041-1723729

Einleitung Die AL-Amyloidose ist eine maligne Systemerkrankung, die unbehandelt rasch letal verläuft. Ein Plasmazellklon bildet Immunglobulin-Leichtketten, die sich im Interstitium zu Fibrillen ablagern. Betroffen sind meist Herz und Nieren, sowie das periphere Nervensystem und selten die Muskulatur.

Methoden Ein 48-jähriger Patient stellte sich mit Verdacht auf eine Motoneuronerkrankung in unserer Klinik vor. 
Ergebnisse Führende Symptome waren eine progrediente generalisierte Muskelschwäche, Dysphagie, Dysarthrie und ein Gewichtsverlust von $20 \mathrm{~kg}$. Zuerst sei allerdings eine Hypästhesie der Extremitäten aufgetreten. Klinisch bestanden eine mittelgradige, proximal betonte Tetraparese sowie eine generalisierte Sensibilitätsstörung aller Qualitäten. Elektromyographisch zeigten sich floride Denervierungszeichen auf 3 Etagen einschließlich paraspinal sowie der Zunge, allerdings bestand neurographisch eine sensomotorische führend demyelinisierende Schädigung. Laborchemisch fand sich eine monoklonale Gammopathie ( $\lg G \lambda)$. Eine Biopsie aus dem M. vastus lat. zeigte Kongorot-positive Ablagerungen, immunhistochemisch positiv für $\lambda$ AL-Amyloid. Es ergaben sich zudem Hinweise auf eine kardiale Beteiligung. Eine Therapie mit Cyclophosphamid, Bortezomib und Dexamethason wurde umgehend eingeleitet.

Diskussion; Die AL-Amyloidose mit neuro- und myopathischer Beteiligung ist eine seltene, aber wichtige, behandlungsbedürftige Differentialdiagnose bei Verdacht auf eine Motoneuronerkrankung.

\section{P 58 Myotonie, Muskelsteifheit und -elastizität bei neuromuskulären Erkrankungen}

\author{
Autoren Lukas $\mathrm{K}^{1}$, Wenninger $\mathrm{S}^{1}$, Schoser $\mathrm{B}^{1}$ \\ Institute 1 Friedrich-Baur-Institut, Neurologie, München, Deutschland \\ DOI 10.1055/s-0041-1723730
}

Einleitung Verschiedene neuromuskuläre Krankheiten gehen mit unterschiedlichen muskulären Symptomen einher. Durch die große Bandbreite an Phänotypen und die variierende Ausprägung der Symptome vergehen bis zur Diagnosestellung häufig mehrere Jahre. In der Regel erfolgt die Diagnose in einer invasiven, nicht selten belastenden Untersuchung. In der Studie wird unter Verwendung eines Myotonometers nicht-invasiv die Muskelspannung, Steifigkeit und Elastizität der Muskelfaser bei unterschiedlichen neuromuskulären Erkrankungen untersucht. Ziel der Studie ist es, Referenzdaten unterschiedlicher neuromuskulärer Erkrankungen mithilfe des Muskeltonusmessgerätes zu gewinnen und seine diagnostische Güte und differentialdiagnostische Abgrenzung zu ermitteln

Material/Methode Im Rahmen einer monozentrisch explorativen Studie erfolgte an 55 neuromuskulär erkrankten Patienten sowie 20 gesunden Kontrollpersonen Messungen mit dem Myotonometer sowie ein 6MWT, eine Myosonografie, eine Muskelkrafteinschätzung anhand der MRC-Skala und eine quantitative Muskelkraftmessung als Vergleichserhebung.

Ergebnisse Präsentation erster Ergebnisse. Aktuell befinden sich die Daten noch in der Auswertung.

Diskussion Anhand der gewonnenen Referenzwerte soll die diagnostische Güte des Muskeltonusmessgeräts bestimmt werden. Normwerte zur einfachen und nicht-invasiven Diagnostik werden erarbeitet und können zur Verlaufsbeurteilung auch unter ggf. Therapie herangezogen werden.

\section{P 59 Prävalenz von Neuropathien bei entzündlich- rheumatischen Erkrankungen}

\begin{abstract}
Autoren Hasseli $\mathrm{R}^{1}$, Tschernatsch $\mathrm{M}^{2,3}$, Heimann $\mathrm{N}^{1,3}$, Ruck $\mathrm{T}^{4}$, Allendörfer $]^{5}$, Gerriets $T^{3,6}$, Müller-Ladner $U^{1}$, Schänzer $A^{7}$

Institutes 1 Campus Kerckhoff, Justus-Liebig-Universität Giessen, Abteilung für Rheumatologie und Klinische Immunologie, Bad Nauheim,

Deutschland; 2 MVZ am Hochwald, Gesundheitszentrum Wetterau gGmbH, Bad Nauheim, Deutschland; 3 Neurologische Klinik, Justus-Liebig-Universität, Gießen, Deutschland; 4 Klinik für Neurologie, Heinrich-Heine-Universität, Düsseldorf, Deutschland; 5 Asklepios Neurologische Klinik, Bad Salzhausen, Deutschland; 6 Stroke Unit Bürgerhospital, Gesundheitszentrum Wetterau gGmbH, Friedberg, Deutschland; 7 Institut für Neuropathologie, Justus-LiebigUniversität, Gießen, Deutschland

DOI 10.1055/s-0041-1723731
\end{abstract}

Einleitung Entzündlich-rheumatische Erkrankungen (ERE) können mit einer Beteiligung des peripheren Nervensystems einhergehen. Unterschiedliche Symptome, wie neuropathische Schmerzen, Sensibilitätsstörungen und
Paresen, können auftreten. Die Prävalenz und die Ursache der Neuropathie (NP) bei ERE ist bisher noch unklar.

Material/Methode In die Studie wurden bislang 22 Patienten mit ERE im medianen Alter von 62,5 Jahren (J) (44-74 J) eingeschlossen, 82 \% sind weiblich, die Erkrankungsdauer liegt im Median bei 9,5 J (3-40 J). 14 Patienten hatten eine Rheumatoide Arthritis (RA), 5 eine Spondylarthropathie (SPA), 3 eine systemische Sklerose (SSc). Das Vorliegen einer Neuropathie wurde klinisch diagnostiziert und mit elektrophysiologischen Messungen weiter in axonale oder demyelinisierende large fibre NP (LFNP) charakterisiert. Eine small-fibre NP (SFN) wurde mittels Hautbiopsie diagnostiziert.

Ergebnisse Eine LFNP war bei $32 \%$ (7/22) der Patienten, eine LFNP mit zusätzlicher SF Beteiligung bei 50 \% (11/22) der Patienten nachweisbar. Die LFNP zeigten sowohl axonale als auch demyelinisierende Schädigungsmuster. Zwei Patienten (9\%) wiesen eine isolierte SFN auf. Bei zwei Patienten (9\%) zeigte sich keine NP.

Diskussion Eine NP war bei in $90 \%$ (20/22) der Patienten mit ERE nachweisbar, wobei eine LFNP überwog. Dieser hohe Anteil an NP bei ERE deutet auf eine überraschend hohe Koinzidenz beider Erkrankungen hin.

\section{P 60 LGMD R9 (= LGMD 2I): Der lange Weg zur Diagnose - ein Fallbericht}

Autoren Glaubitz $\mathrm{S}^{1}$, Kummer $\mathrm{K}^{1}$, Zechel $\mathrm{S}^{2}$, Pauli $\mathrm{S}^{3}$, Schmidt $\mathrm{J}^{1}$ Institutes 1 Universitätsmedizin Göttingen, Neurologie, Göttingen, Deutschland; 2 Universitätsmedizin Göttingen, Neuropathologie, Göttingen, Deutschland; 3 Universitätsmedizin, Humangenetik, Göttingen, Deutschland DOI 10.1055/s-0041-1723732

Einleitung Die LGMD R9 ist eine hereditäre Myopathie mit einem variablen Phänotyp und Manifestationsalter. Die Erkrankten weisen eine progrediente Schulter- und Beckengürtelschwäche auf. Es kann zu einer Kardiomyopathie und respiratorischen Insuffizienz kommen. Ursächlich sind homozygote oder compount heterozygote Mutationen des FKRP-Gens. Das betroffene Protein ist das Fukutin-assoziierte Protein.

Methoden Es erfolgt eine Fallpräsentation eines Patienten, der sich 18 Jahre nach Diagnosestellung einer Polymyositis in unserer Klinik vorstellte.

Ergebnisse Der 59-jährige Patient berichtete von einer progredienten proximalen Muskelschwäche und Myalgien. Vor 18 Jahren wurde auf Grundlage einer CK-Erhöhung, eines grenzwertigen Autoantikörpers (SCl-70) und einer Muskelbiopsie mit Nachweis einer milden Entzündungsreaktion eine Polymyositis diagnostiziert. Eine antiinflammatorische Therapie wurde eingeleitet. Bei der Erstvorstellung bestand die Therapie aus Mycophenolat-Mofetil und Immunglobulinen (30g/alle 4 Wochen). Trotz Therapie kam es zu einem Voranschreiten der Erkrankung, weswegen eine genetische Testung erfolgte, die eine homozygot vorliegende, pathogene Mutation im FKRP-Gen ergab (c.826C>A).

Diskussion Hereditäre Myopathien werden manchmal als Myositis fehldiagnostiziert. Insbesondere eine fehlende Besserung trotz Therapie und unspezifische Entzündungszeichen in der Muskelbiopsie sollten dazu führen, dass die Diagnose einer Myositis im Verlauf kritisch überprüft wird.

\section{P 61 Thinking outside the box - persistent hypokalemia and intermittent muscle weakness}

Authors Rabenstein $\mathrm{M}^{1}$, Abicht $\mathrm{A}^{2,3}$, Brunn $\mathrm{A}^{4}$, Lehmann $\mathrm{H}^{1}$, Wunderlich $\mathrm{G}^{1,5}$ Institute 1 Universität zu Köln, Uniklinik Köln, Neurologie, Köln, Deutschland; 2 Medizinisch Genetisches Zentrum (MGZ) München, München, Deutschland; 3 Friedrich-Baur-Institut, Ludwig-Maximilians-Universität München, Neurologie, München, Deutschland; 4 Universität zu Köln, Uniklinik Köln, Neuropathologie, Köln, Deutschland; 5 Universität zu Köln, Uniklinik Köln, Zentrum für seltene Erkrankungen, Köln, Deutschland DOI 10.1055/s-0041-1723733

Introduction A 22-year old man presented to our neuromuscular outpatients' clinic with recurrent muscular weakness. For the past nine years, he had been 
suffering from muscle pain and weakness mostly after exercise, lasting up to two days. He had also experienced one episode of severe rhabdomyolysis after cold exposure. Additionally, he had a longstanding history of asymptomatic hypokalemia.

Method Case report

Results Electromyography and nerve conduction studies were normal. Muscle biopsy was performed showing a moderately expanded spectrum of muscle fiber size and a pathologically increased lipid storage. The acyl-carnitine spectrum showed slightly elevated levels of malonyl-, palmitoyl- and oleoylcarnitine. These findings seemed to be suspicious for lipid storage/metabolism diseases. Therefore, molecular genetic analysis was performed including ACADVL, CPT1A, CPT2, EFA, ETFB, ETFDH, LPIN1, PYGM, SLC22A5, SLC25A20, but no pathogenic variants could be detected. As next step, variant filtering was extended to further genes taking hypokalemia into account. Thereby, two heterozygous variants were found in the SLC12A3 gene, leading to the diagnosis of Gitelman syndrome.

Discussion This diagnosis explains the combination of longstanding hypokalemia, periodic muscular symptoms, and the episode of rhabdomyolysis. This case highlights the importance of differential diagnostic consideration of hypokalemia as a cause of neuromuscular symptoms not due to muscular channelopathies.

\section{P 62 Die Myotonia congenita Thomsen und der Beitrag von Adolph Seeligmüller aus Halle/Saale}

Autoren Arendt $C^{1}$, Zierz S

Institute 1 Martin-Luther-Universität Halle-Wittenberg, Universitätsklinik und Poliklinik für Neurologie, Halle, Deutschland

DOI 10.1055/s-0041-1723734

Einführung Adolph Seeligmüller war als außerordentlicher Professor an der ehemaligen Vereinigten Friedrichs-Universität Halle-Wittenberg tätig. 1876 erschien eine seiner Publikationen, die dafür sorgte, dass er stellenweise als Erstbeschreiber der Myotonia congenita vor Julius Thomsen angesehen wird. Dennoch ist Seeligmüllers Beitrag zur Myotonia congenita heute weitgehend in Vergessenheit geraten.

Mehtoden Analyse der Publikationen und Biographien Thomsens und Seeligmüllers. Vergleich mit früheren Beschreibungen der Symptomatik.

Ergebnisse Seeligmüllers Publikation erschien kurz nach Thomsen. Er kritisierte daran die Bezeichnung "Ataxie" und die Vermutungen Thomsens zur Pathogenese (Störung “in der einen Thätigkeitshälfte des Gehirns, des Willens") $[1,2]$. Seeligmüller sah die Ursache in einer "schwerer beweglichen Muskelsubstanz" [1].

Schlussfolgerungen Es wäre gerechtfertigt gewesen, aufgrund von Seeligmüllers besserem Verständnis der Pathogenese, seinen Namen in das Eponym aufzunehmen. Bereits Strümpell erklärte, dass Thomsens Ausführungen doch sehr subjektiv, wenn auch zugleich sehr "interessant”, waren.

[1] Seeligmüller A (1876) Tonische Krämpfe in willkürlich beweglichen Muskeln (Muskelhypertrophie?). Dtsch med Wschr 2:389-392, 2:401-403 [2] Thomsen J (1876) Tonische Krämpfe in willkürlich beweglichen Muskeln in Folge von ererbter psychischer Disposition (Ataxia muscularis?). Arch Psychiat Nervenkr 6:702-718

\section{P 63 Novel lysosomal positioning defects due to bi-allelic mutations in BORCS7 causes a neurodegenerative disease presenting as hereditary- spastic paraplegia.}

Authors Daimagüler $\mathrm{H}^{1,2}$, Ardicli $\mathrm{D}^{3}$, Nürnberg $\mathrm{P}^{4}$, Keren-Kaplan $\mathrm{T}^{5}$, Bonifacino $\mathrm{S}^{5}$, Topaloglu $\mathrm{H}^{6}$, Cirak $\mathrm{S}^{1,2}$

Institute 1 Zentrum für Molekulare Medizin, Kinder-und Jugendheilkunde, Köln, Deutschland; 2 Universitätsklinikum Köln, Kinder-und Jugendheilkunde, Köln, Deutschland; 3 Hacettepe University Hospital, Ankara, Türkei; 4 Cologne
Center for Genomics, Köln, Deutschland; 5 Eunice Kennedy Shriver National Institute of Child Health and Human Development, Bethesda, Vereinigte Staaten; 6 Yeditepe University, Istanbul, Türkei DOI 10.1055/s-0041-1723735

B Two multimeric endosomal complexes are known as the regulator for the Iysosomal function and mobility, BLOC-1 and BORC. One of the core subunits of the BORC complex is BORCS7, a 106 amino acid protein with a C-terminal coiled coil domain. A mutation in BORCS7(p.Q87*) in mice displayed impaired motor performance in several tests at 5 weeks of age, accompanied by morphologic abnormalities within the spinal cord with similarities to human Hereditary Spastic Paraplegia (Snouweart, 2018). Nomutations in human BORCS7have been reported until now.

M\&M We recruited an 8-year old boy presenting seizures and spasticity, severe global delay with the inability to speak. Muscle biopsy resembled a severe neurogenic atrophy. To unravel the underlying etiology, we performed Exome Sequencing. Data analysis and variant filtering was performed with Varbank 2 according to an autosomal recessive inheritance.

$\mathrm{R}$ Genomic analysis revealed the first homozygous stop mutation in BORCS7 (c.247C > T, p.Q83*)in this patient, confirmed by co-segregation analysis of the family. Thus is the first report in human of BORCS7 mutations and defines a novel neurodegenerative disease.

$D$ We are experimentally working hypothesis is that the found nonsense mutation in BORCS7 will disrupt the binding to the other subunits of the BORC complex. Thus, a missing core component of the complex, as BORCS7 was reported, will not be able to function as a regulator of the lysosome positioning in neurons.

\section{P 64 Characterization of oropharyngeal dysphagia in idiopathic inflammatory myopathies using FEES - retrospective data on dysphagia pathology and outcome}

Authors Labeit B ${ }^{1}$, Pawlitzki $\mathrm{M}^{1}$, Perlova $\mathrm{K}^{1}$, Ruck $\mathrm{T}^{1}$, Muhle $\mathrm{P}^{1}$, Claus I ${ }^{1}$, Suntrup-Krueger $S^{1}$, Dziewas $R^{1}$, Warnecke $T^{1}$

Institute 1 Klinik für Neurologie mit Institut für Translationale Neurologie Münster, Klinik für Neurologie, Münster, Deutschland

DOI 10.1055/s-0041-1723736

Introduction Oropharyngeal dysphagia is a clinical hallmark of idiopathic inflammatory myopathy (IIM). This study investigated predictors, outcome, and phenomenology of oropharyngeal dysphagia in patients with different types of IIM.

Methods Flexible endoscopic evaluation of swallowing (FEES) videos of 71 IIM patients were retrospectively analysed for salient findings such as bolus spillage, penetration, aspiration, and pharyngeal residue. Based on these findings, dysphagia severity was rated. Regression analyses were performed to investigate demographic and disease-specific predictors of dysphagia severity and pneumonia as outcome-relevant complication of dysphagia. A score is proposed to rate the quality of the endoscopic white-out as a surrogate marker for pharyngeal muscle weakness with consecutive residue.

Results Our analysis revealed no independent predictors of dysphagia severity. Dysphagia severity, however, was an independent predictor for pneumonia, which occurred in $24 \%$ of patients. Pharyngeal residue with risk of postdeglutitive aspiration was the most common dysphagia pattern. Attenuation of the endoscopic white-out was related to residue severity.

Discussion Dysphagia in IIM assessed with FEES is associated with relevant complications such as aspiration pneumonia and must be considered independently of peripheral muscle weakness and disease duration. Swallowing impairment mainly presents with pharyngeal residue. 
Neue therapeutische Prinzipien

\section{P 65 Efficacy and safety results of the avalglucosidase alfa phase 3 COMET trial in late-onset Pompe disease patients}

Author Schoser B ${ }^{1}$

Institute 1 Friedrich-Baur-Institut, LMU München, Neurologische Klinik und

Poliklinik, München, Deutschland

DOI 10.1055/s-0041-1723737

Background Avalglucosidase alfa (Ava) is a recombinant human GAA specifically designed for enhanced M6P-receptor targeting, enzyme uptake, and glycogen clearance.

Method The Phase 3 COMET trial (NCT02782741) compares Ava $(n=51)$ and alglucosidase alfa $(n=49)$ in treatment-naïve late-onset Pompe disease (LOPD). Primary objective: effect on respiratory muscle function (upright forced vital capacity \%predicted [\%FVC]). Secondary/other objectives: functional endurance, inspiratory/expiratory and lower/upper extremity muscle strength, motor function, QoL, and safety.

Results Treatment with Ava resulted in a $2.43 \%$ greater improvement in \%FVC compared to alglucosidase alfa at Week 49. Statistical non-inferiority $(p=0.0074)$ was met; superiority was borderline significant $(p=0.0626)$. Ava treatment resulted in a $30.01 \mathrm{~m}$ greater improvement in the 6-Minute Walk Test and positive results for all other endpoints. Treatment-emergent adverse events (AEs) were reported in $86.3 \%$ of Ava-treated and $91.8 \%$ of alglucosidase alfa-treated participants. 5 participants withdrew, 4 due to AEs, all in the alglucosidase alfa arm. Serious AEs were reported in 8 Ava-treated and 12 alglucosidase alfa-treated participants. Antidrug antibody responses were similar in both groups. High titers and neutralizing antibodies were more common for alglucosidase alfa.

Conclusion Results demonstrate clinically meaningful improvements and a more favorable safety profile in LOPD with Ava compared to alglucosidase alfa.

\section{P 66 Lamotrigin als first-line Therapie bei dystrophen und nicht-dystrophen Myotonien}

Authors Saak $A^{1}$, Schaefer $]^{1}$

Institute 1 Uniklinikum Dresden, Neurologie, Dresden, Deutschland DOI 10.1055/s-0041-1723738

Einleitung Hereditäre Myotonien zählen zu den lonenkanalerkrankungen und können in dystrophe und nicht-dystrophe Myotonien eingeteilt werden. Therapeutisch werden Antikonvulsiva und Antiarrhythmika eingesetzt, wobei bislang nur Mexiletin in der EU als orphan drug zugelassen ist

Methodik Wir untersuchten 6 Patienten mit molekulargenetisch gesicherter hereditärer Myotonie (2 Myotonia congenita Becker, 1 Myotonia congenita Thomsen, 3 Myotone Dystrophie Typ I) bezüglich des Ansprechens auf Lamotrigin zur Linderung myotoner Symptome. Die Beurteilung des Therapieerfolgs erfolgte mittels Selbsteinschätzung der Patienten, klinischem Monitoring der Myotonie und Erhebung der Myotonic Behaviour Scale (MBS)

Ergebnisse Alle Patienten gaben eine Verbesserung ihrer Myotonie unter Lamotrigin bei guter Verträglichkeit an. Der MBS-Score besserte sich um mindestens 1 und bis zu 3 Punkten. Zudem fand sich klinisch eine Abnahme der Myotonie und die Patienten berichteten zudem von einer Besserung begleitender Symptome wie Myalgien, Schluck-/Kau-/Sprachstörungen und transienter Paresen

Diskussion Unsere Fallserie belegt die gute Wirksamkeit und Verträglichkeit von Lamotrigin bei dystrophen und nicht-dystrophen Myotonien. Aufgrund des viel besseren kardialen Nebenwirkungsprofils und des deutlich niedrigeren Preises im Vergleich zu Mexiletin sollte Lamotrigin als first-line Therapie sowohl bei dystrophen als auch bei nicht-dystrophen hereditären Myotonien in Betracht gezogen werden
P 67 Klinische Aspekte vor und nach der Versorgung durch eine Knee-Ancle-Foot-Orthosis (KAFO) mit einem standphasenverriegelndem Kniegelenk (SCO) bei Post-Polio-Syndrom Patienten

Autoren Ruetz $\mathrm{A}^{1}$

Institute 1 Katholisches Klinikum Koblenz·Montabaur, Klinik für Konservative Orthopädie und des Poliozentrums, Sportmedizinischen U-Stelle des LSB Rhl.-

Pfalz, Koblenz, Deutschland

DOI 10.1055/s-0041-1723739

Das Post-Polio-Syndrom ist eine seltene Erkrankung postinfektioneller Genese, die sich durch Müdigkeit, Muskel- und Gelenkschmerzen sowie Schwäche äußert und meist erst Jahrzehnte nach der eigentlichen Polioinfektion auftritt. Die konservative Orthopädie versorgt Post-Polio-Patienten mit Orthesen der betroffenen Extremitäten, meist den Beinen, um die Mobilität der Betroffenen zu gewährleisten und Degeneration zu verhindern.

Die jahrelang von der Orthopädie eingesetzten orthopädischen Hilfsmittel darunter auch mechanischen Orthesen erhalten seit einiger Zeit eine Weiterentwicklung mit elektromechanischer Technik. Diese Studie zog einen Vergleich zwischen vor und nach Versorgung im Hinblick auf Alltagsfaktoren.

Um die Versorgungstypen möglichst alltagsnah vergleichen zu können, wurden zur Testung je ein 6 Minuten Gehtest vor und einer nach der "Neurotronic-Versorgung" durchgeführt. Das Augenmerk lag während des Tests in den Nebenkriterien auf der Gehstrecke, Herzfrequenz und der Borg-Skala, sowie in den Hauptkriterien auf der Doppelschrittdauer sowie -länge der Ganglinienbreite und der effektiven Fußlänge. Um optimal auf den Test vorbereitet zu sein, nahm jeder der Patienten an einer qualtitativen Gangschule durch Physiotherapeuten der Klinik teil. Erfüllte ein Patient nicht die von uns gestellten Einschlusskriterien, oder nahm nicht an der Gangschulung teil, so wurde er aus der Studie ausgeschlossen.

Bei der Untersuchung von 42 Patienten konnte eine sinkende Wegstrecke (-13,3\%), einen erhöhten Herzfrequenzanstieg (+1,31 bpm Ruhe/Belastung sowie $+1,19$ bpm Ruhe/Nachbelastung) und eine erhöhte Belastungseinschätzung nach Borg (+1,29 Ruhe/Belastung sowie $+0,88$ Ruhe/Nachbelastung) festgestellt werden.

Im Bereich der Hauptaspekte konnte eine verlängerte Doppelschrittdauer $(+4,7 \%)$ sowie Doppelschrittlänge $(+5,3 \%)$ festgestellt werden, sowie eine Zunahme der Ganglinienbreite und eine verlängerte effektive Fußlänge. Signifikant nachweisbar waren dabei die Wegstreckenveränderung, die ansteigende Borg Skala bei dem Vergleich Ruhe/Belastung sowie die Doppelschrittdauer und die Ganglinienbreite.

Es war zu belegen, dass die Orthese den Gang innerhalb gewisser Aspekte sichert, allerdings nicht ökonomisiert. Deswegen sollten weitere Untersuchung die Effizienz der elektromechanischen Orthese erforschen und so den Benefit für den des Patienten objektivieren.

Koblenz, den 15.11.2020

\section{P 68 Somatisches Gen-Editing verbessert Skelett- und Herzmuskelversagen bei translationalen Modellen der Duchenne Muskeldystrophie}

Autoren Krause $S^{1}$, Reichert $S^{1}$, Fonteyne $\mathrm{LM}^{2}$, Moretti $\mathrm{A}^{3}$, Giesert $\mathrm{F}^{4}$, Wurst $\mathrm{W}^{4}$, Wolf $E^{2}$, Kupatt $\mathrm{C}^{3}$, Walter $\mathrm{MC}^{1}$

Institutes 1 Friedrich-Baur-Institut, Ludwig-Maximilians-Universität, München, Deutschland; 2 Molekulare Tierzucht und Biotechnologie, Genzentrum der LMU, München, Deutschland; 3 Klinik und Poliklinik für Innere Medizin I, Klinikum rechts der Isar, Technische Universität München und Deutsches Zentrum für Herz-Kreislauf-Forschung (DZHK), München,

Deutschland; 4 Institut für Entwicklungsgenetik, Helmholtz Zentrum München und Munich School of Life Sciences Weihenstephan, Technische Universität München, München, Deutschland DOI 10.1055/s-0041-1723740 
Das somatische Gen-Editieren durch sequenzspezifische Nukleasen bietet neue Möglichkeiten, bei Duchenne Muskeldystrophie das Leseraster des Dystrophin-Gens wiederherzustellen, was zur Expression eines verkürzten, aber weitgehend funktionsfähigen Dystrophin-Proteins führt. In einem Schweinemodell der DMD (Deletion von Exon 52, DMD $\Delta 52$ ) sowie in einem entsprechenden, vom Patienten abgeleiteten Modell induzierter pluripotenter Stammzellen wurde durch intramuskuläre Injektion bzw. Transduktion mit adeno-assoziierten viralen Vektoren des Serotyps 9, die Expression eines verkürzten Dystrophins induziert (DMD $\Delta 51-52)$.

Die wiederhergestellte Expression von membrangebundenem DMD $\Delta 51-52$ nach G2-AAV9-Cas9-gE51-Gabe führte zu erhöhten Konzentrationen von membrangebundenem $\gamma$-Sarkoglykan und $\beta$-Dystroglykan und zur Wiederherstellung des DGC. Es zeigte sich eine verbesserte Skelettmuskelstruktur mit verringertem Kapillarverlust, verminderter mononukleärer Zellinfiltration und interstitieller Fibrose. Mittels iPSCs war es möglich, die Auswirkungen der menschlichen DMD $\Delta 52$ Mutation zu modellieren und die Wirksamkeit der AAV-Cas9-vermittelten somatischen Exzision von Exon 51 in menschlichen Muskelzellen zu evaluieren, die theoretisch etwa $14 \%$ aller DMD-Mutationen korrigieren könnte.

Die Wirksamkeit der Cas9-vermittelten Exon-Exon-Exzision, bereitet den Weg für neue Behandlungsansätze bei Patienten mit Muskeldystrophie Duchenne.

\section{P 69 In vitro und in vivo Untersuchungen zur muskulären Aufnahme und Aktivität von in Moos produziertem, nicht phosphoryliertem rhGAA (Moss-GAA)}

Autoren Hintze $S^{1}$, Dabrowska-Schlepp $P^{2}$, Berg $B^{2}$, Busch $A^{2}$, Schaaf $A^{2}$, Meinke $\mathrm{P}^{1}$, Schoser $\mathrm{B}^{1}$

Institutes 1 Friedrich-Baur-Institut/Klinikum der Universität München, AG MDRU, München, Deutschland; 2 eleva GmbH, Freiburg, Deutschland DOI 10.1055/s-0041-1723741

Einleitung Morbus Pompe ist eine autosomal rezessive Glykogenspeichererkrankung, die durch fehlende oder verminderte Aktivität des lysosomalen Enzyms $\alpha-1,4$-Glucosidase (GAA) verursacht ist. Die Erkrankung führt zu einer pathologischen Anreicherung von Glykogen im Gewebe was zu Schäden in Zellorganellen, Zellen und Organen führt und wovon Herz und Skelettmuskulatur am stärksten betroffen sind. Die einzige verfügbare Therapie für Morbus Pompe ist eine Enzymersatztherapie (ERT), bei welcher rekombinantes humanes GAA (rhGAA) über den Mannose-6Phoshat-Rezeptor in Lysosomen aufgenommen wird.

Ein unmet-medical-need dieser Therapie ist die Verbesserung der Enzymaufnahme in Skelettmuskulatur.

Material/Methode Wir haben in Moos produziertes, nicht-phosphoryliertes rhGAA (Moss-GAA) in Muskelzellkulturen und Gaa-/- Mäusen hinsichtlich der Aufnahme in Zellen bzw. Organe getestet. Um Aufnahme und Effizienz zu testen haben wir einen GAA-Aktivitätsassay und einen quantitativen GlykogenNachweis verwendet.

Ergebnisse Wir können zeigen das Moss-GAA in vitro in Muskelzellen sowie in vivo in Muskulatur aufgenommen wird, aktiv ist, und eine Prozessierung zum reifen Protein erfolgt.

Diskussion Die Aufnahme und Prozessierung von nicht-phosphoryliertem rhGAA in Skelettmuskulatur erweitert die Therapiemöglichkeiten des Morbus Pompe da somit möglicherweise der Engpass der Aufnahme via des Mannose-6-Phoshat-Rezeptors in humanen Skelettmuskeln umgangen werden kann.

\section{P 70 A Phase I/II Open-label gene replacement - clinical study for late onset pompe disease}

Authors Wenninger $\mathrm{S}^{1}$, Day $\mathrm{JW}^{2}$, Mozaffar $\mathrm{T}^{3}$, Schoser B ${ }^{1}$, Foo CWP ${ }^{3}$, Bachtell $\mathrm{N}^{4}$, Conner $\mathrm{E}^{4}$

Institute 1 Friedrich-Baur-Institut/LMU Munich, Neurologische Klinik und Poliklinik, München, Deutschland; 2 Stanford University, Palo Alto, CA, Vereinigte Staaten; 3 University of California, Irvine, CA, Vereinigte Staaten; 4 Audentes Therapeutics Inc., San Francisco, CA, Vereinigte Staaten DOI 10.1055/s-0041-1723742

Pompe disease, an autosomal recessive neuromuscular disease caused by mutations in the GAA gene leading to functional enzyme acid alpha-glucosidase (GAA) deficiency. Late-onset Pompe disease (LOPD) is characterized by progressive skeletal myopathy with muscle weakness and respiratory insufficiency, leading to fulltime ventilator dependence, wheelchair use and early death. AT845, an AAVbased gene therapy for Pompe disease under development is designed to target muscle tissue. Preclinical studies in GAAKO mice infused with AT845 have resulted in GAA activity ranging from $25 \%$ to $500 \%$ of wild-type levels in muscle at the clinical starting dose of $3 \times 1013 \mathrm{vg} / \mathrm{kg}$. Administration of AT845-cyno construct expressing the macaque GAA in Macaca fascicularis monkeys resulted in increased expression of GAA in muscle with no detectable immune response or tissue toxicity compared to contols. A one-time infusion of AT845 has the potential for stable, long-term sustained expression of GAA in muscle with therapeutic benefit. FORTIS (NCT04174105), a Phase I/II, multicenter, open-label, ascending-dose clinical study is assessing the safety and preliminary efficacy of AT845 in up to 8 LOPD subjects $\geq 18$ years. Study subjects will receive a single i.v. infusion of AT845 with 1 year of follow-up, monitoring frequently endpoints including GAA activity and protein level in muscle. Subjects will then be followed for 4 years to determine duration of response and long-term effects.

\section{P 71 NCAM knock-out als einfaches und rasches read- out System zur Evaluierung von CRISPR/Cas-basiertem Gene-Editing in primären humanen Myoblasten}

Autoren Stadelmann $C^{1,2}$, Marg $A^{2}$, Di Francescantonio $S^{2}$, Spuler $S^{1,2,3}$, Fernandez $\mathrm{HE}^{1,2}$

Institutes 1 Max-Delbrück Center for Molecular Medicine, Berlin, Deutschland; 2 Experimental and Clinical Research Center, Muscle Research Unit, Berlin, Deutschland; 3 Charité Universitätsmedizin, Berlin, Deutschland DOI 10.1055/s-0041-1723743

Fragestellung Gene-Editing über molekulare Werkzeuge, wie das traditionell verwendete SpCas9 oder die neuartigeren Base Editors, gehören zu den vielversprechenden, womöglich sicheren Methoden der Genkorrektur, die durch die Entdeckung der CRISPR/Cas Systeme möglich werden. Monogenetische Muskeldystrophien könnten durch diese Neuerungen therapierbar werden. Um Genkorrektur-Strategien zu entwickeln, benötigen wir passende, muskelspezifische Analyse-Möglichkeiten. NCAM ist ein in Myoblasten exprimiertes Oberflächenprotein, dessen knock-out zur Evaluation von Gene-Editing Werkzeugen nützlich sein kann.

Methoden Primäre humane Myoblasten (100\% Desmin-positiv) wurden transfiziert mit verschiedenen guide RNAs die in ein Plasmid kloniert wurden, das, zusammen mit der Gen modifizierenden Nuklease, einen Venus-Reporter enthält. Der NCAM-knockout wurde analysiert durch Sequenzierung, Immunfärbung und Durchflusszytometrie (FACS).

Ergebnisse Der knock-out von NCAM konnte erfolgreich in primären humanen Myoblasten generiert werden, wie durch Sequenzierung und FACS Analyse gezeigt.

Schlussfolgerung Mit dem effektiven und schnell analysierbaren NCAM-readout haben wir ein wichtiges Werkzeug geschaffen, das CRISPR/Cas-basierte Werkzeug z.B auf mRNA oder Protein-Basis, zu evaluieren und damit den translationalen Prozess deutlich zu beschleunigen. 


\section{P 73 Generierung von hiPS Zellen aus Muskelbiopsien als Basis für die Produktion einer unbegrenzten Anzahl induzierter myogener Zellen}

Autoren Metzler $\mathrm{E}^{1,2}$, Fernandez $\mathrm{HE}^{1,2}$, Sunaga-Franze $\mathrm{DY}^{2,3}$, Sauer $\mathrm{S}^{2,3}$, Diecke $\mathrm{S}^{3,4}$, Spuler $\mathrm{S}^{1,2,5}$

Institutes 1 Experimental and Clinical Research Center, a joint cooperation of Charité, Universitätsmedizin Berlin and the Max Delbrück Center for Molecular Medicine in the Helmholtz Association (MDC), Muscle Research Unit, Berlin, Deutschland; 2 Max Delbrück Center for Molecular Medicine in the Helmholtz Association (MDC), Berlin, Deutschland; 3 Berlin Institute of Health (BIH), Berlin, Deutschland; 4 Pluripotent Stem Cells Platform, Max Delbrück Center for Molecular Medicine in the Helmholtz Association (MDC), Berlin,

Deutschland; 5 Charité Universitätsmedizin, Berlin, Deutschland

DOI 10.1055/s-0041-1723744

Muskelbiopsien stellen eine notwenige Prozedur in der Diagnostik von muskeldegenerierenden Erkrankungen dar, während gleichzeitig zell- und gentherapeutische Ansätze unter Verwendung von Muskelstammzellen, den sogenannten Satellitenzellen, mehr und mehr an Bedeutung gewinnen. Der Einsatz von Satellitenzellen ist jedoch durch eine begrenzte Zellzahl limitiert. Muskelzellen aus Biopsien könnten ebenso zur Generierung von humanen induzierten pluripotenten Stammzellen (hiPSCs) verwendet werden, welche den Schlüssel zu einer uneingeschränkten Anzahl autologer Zellen darstellen, die zur Regeneration von großen Muskeln benötigt werden. Daher haben wir in dieser Arbeit hiPSCs aus primären Muskelzellen generiert und diese mit hiPSCs aus peripheren mononukleären Blutzellen (PBMCs) desselben Donors $(n=4)$ verglichen. Unsere Ergebnisse zeigen eine 40x höhere Reprogrammierungseffizienz für Myoblasten im Vergleich zu PBMCs. RNA-Seq Analysen ergaben dabei nur geringe Unterschiede zwischen den beiden Zelltypen, und das myogene Differenzierungspotential war identisch. Anschließende Transplantationsversuche zeigten zudem für beide Zelltypen das Potential zur Muskelfaserregeneration. Wir kommen zu dem Schluss, dass Muskelbiopsie-Proben eine sinnvolle und nützliche Quelle für die Generierung von hiPSCs darstellen mit dem Potential für die Verwendung in zukunftsweisenden Therapieansätze.

\section{P 74 Gene Editing in LMNA related Muscular Dystrophy Patient-derived Cells}

\author{
Authors Wang $\mathrm{H}^{1}$, Müthel $\mathrm{S}^{1}$, Marg $\mathrm{A}^{1}$, Fernandez $\mathrm{HE}^{1}$, Krause $\mathrm{A}^{1}$, \\ Spuler $\mathrm{S}^{1,2}$ \\ Institute 1 Max-Delbrück-Centrum für Molekulare Medizin, ECRC, Berlin, \\ Deutschland; 2 Charité, Berlin, Deutschland \\ DOI 10.1055/s-0041-1723745
}

Laminopathy comprises a set of diverse rare diseases genetically caused by mutations in genes coding for nuclear lamina. Classical laminopathy refers to diseases caused by mutations in gene LMNA coding for lamin A/C, key components of nuclear lamina. The majority of classical laminopathy are caused by autosomal dominant mutations and the clinical phenotypes vary from muscular dystrophy, dilated cardiomyopathy, Charcot-Marie-Tooth type 2B, to aging phenotype progeria. Currently, there is no cure for laminopathies.

To improve the muscle condition of patients with LMNA related muscular dystrophy, we aim to conduct transplantation therapy with patient-derived muscle stem cells (MSC) after genetic correction. To achieve this, we first screen for efficient editing methods among the state of the art gene editing techniques including allele specific CRIPSR-Cas9 or CRISPR base editing in patient-derived induced pluripotent stem cells (iPSC). Further the validated editing strategy from iPSC is applied to patient-derived MSC to prepare material for the transplantation therapy. Meanwhile to assess the correction effects, evaluations of lamin $\mathrm{A} / \mathrm{C}$ on expression, nuclear localization and cellular functions such as mitosis are performed with edited, non edited and healthy control cells. Future work will expand on more LMNA mutations of different patients with personalized gene editing strategy to provide a more generalized transplantation therapy option to classical laminopathy.

Stratifizierung und Behandlungskohorten

\section{P 75 Untersuchungen des Zytoskeletts von sensorischen Nervenfaserendigungen in Hautbiopsien von Motoneuronerkrankten}

\author{
Autoren Rödiger $A^{1}$, Vozobulova $M^{2}$, Metzner $K^{1}$, Steinbach $R^{1}$, Gaur $N^{1}$, \\ Axer $\mathrm{H}^{1}, \overline{\text { Brill MS }^{2}}$, Grosskreutz $]^{1}$ \\ Institutes 1 Universitätsklinikum Jena, Klinik für Neurologie, Jena, \\ Deutschland; 2 Technische Universität München, Institut für Zellbiologie des \\ Nervensystems, München, Deutschland \\ DOI 10.1055/s-0041-1723746
}

Einleitung In Motoneuronerkrankungen sind die kausalen molekularen Mechanismen größtenteils unverstanden, die zu neuroaxonalen Störungen und Motoneuronverlust führen. Hier analysieren wir sensorische Nervenfaserendigungen, die in Hautbiopsien zu finden sind. Ähnlich wie Motoneurone, haben sensorische Neurone lange Fortsätze (Weis et al., 2011). Wir fokussieren uns hier auf das Zytoskelett, da NfL ein bekannter Biomarker der ALS ist.

Methode Oberschenkelhautbiopsien von einer kleinen initialen Kohorte (3 SMA-, 7 ALS-Patienten, 4 Gesunde) wurden immunhistochemisch gefärbt ( $\beta$ IIITubulin, nicht-phosphoryliertes/phosphoryliertes Neurofilament $\mathrm{NfH}$ : npNfH/ pNfH), konfokale Bilder der Dermis aufgenommen und die Grauwerte quantitativ analysiert.

Ergebnisse Die Grauwerte der Immunfluoreszenzfärbungen zeigen einen Trend in ALS-Patienten anzusteigen, während sie in SMA Patienten abfallen ( $\beta$ III-Tubulin, $p=0,07$; npNfH, $p=0,117$ und pNfH, $p=0,07$ ).

Diskussion Hautbiopsien von Patienten mit Motoneuronerkrankungen könnten sich als neuartiges "pathogenes Fenster" erweisen und einerseits zur Diagnose, andererseits zur Analyse von bekannten oder neuen Biomarkern verwendet werden. In den nächsten Schritten werden wir die Längenabhängigkeit dieses Effektes studieren (Ober- vs. Unterschenkel) und auf mehr Biomarker färben. Hautbiopsien könnten langfristig dazu dienen, Therapieerfolge zu analysieren oder neue Therapieansätze zu entwickeln, da sie wiederholt durchführbar sind.

\section{P 76 Pain assessment and characterization in Charcot-Marie-Tooth Disease (CMT)}

Authors Bartl $\mathrm{M}^{1}$, Reinecke $\mathrm{L}^{1}$, Hirsch $]^{1}$, Prukop $\mathrm{T}^{1,2}$, Sonja $\mathrm{F}^{3}$, Krahn $\mathrm{AB}^{1}$, Wigge $\mathrm{S}^{4}$, Sereda $\mathrm{MW}^{1,2}$

Institute 1 University Medical Centre Göttingen, Department of Neurology, Göttingen, Deutschland; 2 Max-Planck-Institute for Experimental Medicine,

Department of Neurogenetics, Göttingen, Deutschland; 3 Asklepios

Fachklinikum, Psychiatrie, Tiefenbrunn, Deutschland; 4 Grünenthal GmbH, Aachen, Deutschland

DOI 10.1055/s-0041-1723861

Introduction Charcot-Marie-Tooth (CMT) is the most common hereditary neuropathy. Clinically, patients show high variability including symmetric paresis, sensory loss, foot deformities and areflexia. Pain is an important symptom, but its extent, frequency, character and impact on Quality of Life (QoL) or Activities of Daily Living (ADL) has so far not been sufficiently assessed.

Methods In a survey study via the German CMT network (CMT-NET), pain and its characteristics in CMT was documented using the standardized questionnaires painDETECT and NPSI to screen for neuropathic pain and SF-36 to evaluate QoL impairment.

Results Of 200 subjects with molecularly diagnosed CMT (76 \% CMT1A, $10.5 \%$ CMTX, 6.5\% HNPP, 4\% CMT1B, 3\% CMT2A), 33\% were on pain medication, mostly non-steroidal anti-inflammatory drugs. Pain had a prevalence of $55 \%$ which increased with age. Surprisingly, $52 \%$ of the patients had nociceptive pain and only $24 \%$ displayed neuropathic pain, which, however, had a higher 
intensity. Moderate but chronic pain was most frequent and associated with QoL reduction and $\mathrm{ADL}$ impairment. $\mathrm{PDQ} / \mathrm{PSI}$ values correlated well with each other. Discussion Pain is a common and relevant symptom in CMT with mostly nociceptive features. The number of patients taking pain medication was lower as expected, indicating an urge for treatment optimization. Extended and longitudinal studies are needed to elucidate pain characteristics and treatment effects over time and in different subtypes.

\section{P 77 Plasma-1-Desoxy-sphingolipid-Spiegel und (para-) klinische Korrelationen bei 100 Patienten mit idiopathischer Small Fiber Neuropathie}

Autoren Dumke $C^{1}$, Hornemann $\mathrm{T}^{2}$, Nikolin $\mathrm{S}^{3}$, Kurth $\mathrm{I}^{4}$, Ouwenbroek $\mathrm{A}^{1}$, Schulz JB ${ }^{1}$, Gess $B^{1}$, Rolke ${ }^{5}$, Dohrn MF $^{1}$

Institutes 1 Uniklikum der RWTH Aachen, Klinik für Neurologie, Aachen, Deutschland; 2 Universitätsspital Zürich, Institut für Klinische Chemie, Zürich, Schweiz; 3 Uniklinikum der RWTH Aachen, Institut für Neuropathologie, Aachen, Deutschland; 4 Uniklinikum der RWTH Aachen, Institut für Humangenetik, Aachen, Deutschland; 5 Uniklinikum der RWTH Aachen, Abteilung für Palliativmedizin, Aachen, Deutschland

DOI 10.1055/s-0041-1723862

Einleitung Small Fiber Neuropathien (SFN) betreffen selektiv die kleinen (un-) myelinisierten Nervenfasern und manifestieren sich mit Schmerzen, Sensibilitätsstörungen und autonomen Symptomen. 1-desoxy-Sphingolipide (dSL) entstehen, wenn bei der Synthese von Sphinganin und Sphingosin statt L-Serin LAlanin verstoffwechselt wird. Durch Störung des axonalen Aussprossens wirken sie neurotoxisch.

Material/Methode Bei 100 Patienten mit idiopathischer SFN korrelierten wir Anamnese, neurologische Untersuchung, Quantitativ Sensorische Testung (QST), sudomotorische Funktion und intraepidermale Nervenfaserdichte (IENFD) mit dem Aminosäure- und Sphingolipidprofil. Ein Diabetes mellitus und pathogene Varianten in SPTLC1 und SPTLC2 wurden ausgeschlossen.

Ergebnisse Plasma-dSL-Spiegel korrelierten invers mit der IENFD, aber nicht mit funktionellen Markern der Small Fiber Dysfunktion. Als Ausdruck einer A $\beta$ Dysfunktion korrelierte die mechanische Detektionsschwelle mit den 1-Desoxysphinganin-Spiegeln. Bei Patienten mit Übergewicht, Bluthochdruck oder Fettstoffwechselstörung waren die dSL-Spiegel signifikant höher. Es bestand eine inverse Korrelation mit den L-Serin-Spiegeln.

Diskussion Unsere Daten deuten darauf hin, dass metabolische Risikofaktoren über erhöhte dSL-Plasmaspiegel zur Nervenfaserdegeneration beitragen und die Entstehung von SFN begünstigen.

\section{P 78 Diagnose SMA im Neugeborenenscreening - was bedeuten 2 SMN2 Kopien für die Prognose?}

Autoren Vil $K^{1}$, Schwartz $\mathrm{O}^{1,2}$, Blaschek $A^{1}$, Schara-Schmidt $\mathrm{U}^{3}$, MüllerFelber $\mathrm{W}^{1}$, Kölbel $\mathrm{H}^{3}$

Institutes 1 Dr. v. Haunersches Kinderspital der Universität München,

Neuropädiatrie, München, Deutschland; 2 Muenster University Hospital, Department of Pediatric Neurology,, Münster, Deutschland; 3 University of Essen, Department of Pediatric Neurology, Developmental Neurology and Social Pediatrics, Essen, Deutschland

DOI 10.1055/s-0041-1723863

Nach > 2,5 Jahren klinischer Nachbeobachtung von 57 durch Neugeborenen Screening (NGS) identifizierten Patienten mit SMA in Deutschland hat sich gezeigt, dass fast die Hälfte der Patienten mit 2 SMN2 Kopien im Alter von wenigen Lebenstagen Zeichen eines bereits floriden Krankheitsprozesses haben.

10 von 23 Patienten mit 2 SMN2-Kopien wurden als symptomatisch eingestuft. 4 hatten klinische Symptome bei der Erstuntersuchung, 5 entwickelten eine Muskelschwäche im Alter von 2-4 Wochen. Die Ulnaris-CMAP-Amplituden all dieser Kinder lagen zwischen 0 und 1,3 mV. Ein Kind hatte pathologische CMAP-Amplituden ohne klinische Zeichen.
21 Kinder mit 2 SMN2-Kopien wurden ab dem Alter von 14-39 Tagen mit Nusinersen behandelt. Von Anfang an asymptomatische Patienten blieben im Verlauf symptomfrei. Die früh symptomatischen Patienten hatten einen unterschiedlichen Verlauf. Es zeigten sich teils myosonographisch nachweisbare Umbauvorgänge und einige erreichten motorische Meilensteine mit erheblicher Verspätung. Ein Patient entwickelte eine Schluckstörung und ist nachts nicht-invasiv beatmet.

Zusammenfassend findet sich bei einem erheblichen Teil der Patienten mit 2 SMN2-Kopien, die im NGS entdeckt werden, trotz frühester Behandlung ein florider, zum Teil bereits irreversibler Motoneuron-Schaden. Dies macht deutlich, dass Diagnosebestätigung innerhalb weniger Tage und die umgehende Behandlung ist in dieser Gruppe absolut kritisch sind. Der diagnostische Wert der CMAPs ist relevant.

\section{P 79 Diagnose SMA mit 4 SMN2 Kopien im Neugeborenenscreening - behandeln oder nicht?}

Autoren Vill $K^{1}$, Schwartz $\mathrm{O}^{1,2}$, Blaschek $A^{1}$, Schara-Schmidt $U^{3}$, MüllerFelber $\mathrm{W}^{1}$, Kölbel $\mathrm{H}^{3}$

Institutes 1 Dr. v. Haunersches Kinderspital der Universität München, Neuropädiatrie, München, Deutschland; 2 Muenster University Hospital, Department of Pediatric Neurology,, Münster, Deutschland; 3 University of Essen, Department of Pediatric Neurology, Developmental Neurology and Social Pediatrics, Essen, Deutschland

DOI 10.1055/s-0041-1723864

Zwischen Januar 2018 und November 2020 wurden 57 Patienten mit SMA durch Neugeborenenscreening-Projekte in Deutschland identifiziert. Bei einem Drittel lagen $\geq 4$ SMN2 Kopien vor. Die Inzidenz der Erkrankung in der Screeningpopulation lag nicht über den bekannten Zahlen der Allgemeinbevölkerung. International besteht noch Uneinigkeit bezüglich des Vorgehens in dieser Gruppe.

Bei 19 Patienten mit $\geq 4$ SMN2 Kopien wurde eine medikamentöse Therapie versus einer konservativen Strategie unter engmaschigen Kontrollen mit den Eltern, unter steter Berücksichtigung neuer Empfehlungen und Erkenntnisse, fortlaufend diskutiert.

4 Familien haben sich für einen präsymptomatischen Beginn mit Nusinersen entschieden, um das Risiko einer gegebenenfalls irreversiblen Motoneuronschädigung zu minimieren. Eine positive Familienanamnese hatte erheblichen Einfluss auf die Therapieentscheidung.

Bei einem unbehandelten Kind mit 4 SMN2-Kopien wurde eine SMA Typ 3 im Alter von 2 Jahren manifest. Klinisch imponierte eine Verschlechterun der Ausdauer und Stolperneigung (bei erhaltenen Musekleigenreflexen und unauffälligen CMAPs), im EMG bestätigte sich ein neurogenes Muster. In 2 weiteren Familien wurden Geschwister im Vorschulalter mit bislang misinterpretierten Symptomen einer SMA Typ 3 identifiziert. Ein Kind hatte eine im Kindesalter an SMA Typ 3 erkrankte Tante.

Diese Daten sprechen dafür, auch im Kollektiv mit 4 SMN2 Kopien eine frühe Behandlung zu favorisieren.

\section{P 80 Patientenregister für seltene neuromuskuläre Erkrankungen: Nutzen und Erfordernisse - Gemeinsame Anstrengungen zur Etablierung einer besseren Datenlage}

Autoren Thiele $\mathrm{S}^{1}$, Krause $\mathrm{S}^{1}$, Heidemann $\mathrm{M}^{1}$, Greckl $\mathrm{E}^{1}$, Reilich $\mathrm{P}^{1}$, Walter $\mathrm{MC}^{1}$

Institute 1 Friedrich-Baur-Institut, Neurologische Klinik und Poliklinik, Klinikum der Ludwig-Maximilians-Universität München, München, Deutschland

DOI 10.1055/s-0041-1723865

Das am Friedrich-Baur-Institut geführten Register für seltene neuromuskuläre Erkrankungen sind im Rahmen des Projekts TREAT-NMD, einem europaweiten Netzwerk von Ärzten und Forschern für seltene neuromuskuläre 
Erkrankungen, entstanden, um die Diagnose und Therapie für diese Patienten voranzubringen. Vor Zulassung eines neuen Medikaments haben Patienten nur über eine Studienteilnahme Zugang zu therapeutischen Innovationen. Gerade bei seltenen Erkrankungen mit dringendem Bedarf an neuen, effektiven Therapien ist es für Betroffene daher sehr wichtig, an Studien teilzunehmen zu können. Eines der Hauptziele der Registers ist es, im Sinne einer "Trial Readiness" Patienten gezielt zu informieren, wenn sie für eine klinische Studie in Betracht kämen. Wir halten "unsere" Registerpatienten auch über den neuesten Forschungsstand informiert: Dazu versenden wir regelmäßig einen Patientennewsletter. Zusätzlich konnten wir in internationaler Zusammenarbeit neue Behandlungsleitlinien definieren, die wir an registrierte Patienten weitergeben, damit jeder die heutige bestmögliche Therapie erhält. Darüber hinaus ist es auch möglich, Genotyp-Phänotyp Korrelationen, Fragen zu Erkrankungskosten und -belastung, Lebensqualität oder Fragen zu Schwangerschaft bei neuromuskulären Patienten aus Forschungsgesichtspunkten zu untersuchen.

\section{P 81 Getting ready for clinical trials - globale, multizentrische Studie zur detaillierten Genotyp-Phänotyp -Charakterisierung der SORD- Neuropathie}

Authors Dohrn $\mathrm{MF}^{1,2}$, Cortese $\mathrm{A}^{2,3}$, Rebelo $\mathrm{A}^{2}$, Obermaier $\mathrm{CD}^{4}$, Biskup $\mathrm{S}^{4}$, Consortium $\mathrm{IN}^{5}$, Shy $\mathrm{M}^{6}$, Züchner $\mathrm{SL}^{2}$

Institute 1 Uniklinikum der RWTH Aachen, Neurologie, Aachen,

Deutschland; 2 Dr. John T. Macdonald Foundation, Department of Human

Genetics and John P. Hussman Institute for Human Genomics, University of Miami, Miller School of Medicine, Miami, FL, Vereinigte Staaten; 3 Department of Neuromuscular Disease, UCL Queen Square Institute of Neurology and The National Hospital for Neurology, London, Vereinigtes Königreich; 4 Praxis für Humangenetik Tübingen \& CeGaT GmbH, Tübingen, Deutschland; 5 Rare Diseases Clinical Research Network, lowa City, IA, Vereinigte Staaten; 6 University of lowa, Carver College of Medicine, Neurologie, lowa City, IA, Vereinigte Staaten

DOI 10.1055/s-0041-1723866

Einleitung Das Gen SORD kodiert für das Enzym Sorbitol-Dehydrogenase, das Sorbitol in Fruktose umwandelt. Biallelische loss-of-function Mutationen führen zu einer axonalen, führend motorischen Neuropathie (CMT2 oder dHMN). Zukünftige Therapieansätze stellen Aldosereduktaseinhibitoren dar, antisense Medikamente oder Vektor-basierte Gentherapien.

Methoden Planung einer globalen Natural Course Studie zur Identifizierung betroffener Mutationsträger, zur Erfassung des phänotypischen Spektrums sowie Definition von Biomarkern und klinischen Outcome-Parametern. Kollegen, Patienten und Patientenorganisationen sind eingeladen, uns direkt zu kontaktieren.

Ergebnisse Seit November 2020 werden weltweit Patientinnen und Patienten mit axonaler, führend motorischer Neuropathie und autosomal rezessivem oder sporadischem Auftreten genetisch untersucht. Die häufigste Mutation des SORD-Gens ist die Deletion c.757delG; p.Ala253GInfsTer27, die per Sanger-Sequencing gezielt gescreened werden kann. Alternativ stehen bereits NGS-basierte Panel und Exome mit Anreicherung des SORDGens zur Verfügung. In Positivfällen werden anamnestische, klinische, elektrophysiologische und bildgebende Daten gesammelt. Sorbitol-Serumspiegel werden als Biomarker etabliert. Ziel ist es, weltweit mindestens 150 Fälle zu charakterisieren.

Diskussion Diese Studie dient der Vorbereitung von Phase 2 Clinical Trials (study readiness). Betroffene könnten neben der Diagnosestellung auch von neuen Therapien profitieren.

\section{P 82 Deep phenotyping of CMT1A patients in a longitutdinal prospective multicenter study within the German Charcot-Marie-Tooth Disease network (CMT-NET)}

Authors Krahn $\mathrm{AB}^{1}$, Leha $\mathrm{A}^{2}$, Sonja $\mathrm{F}^{3}$, Prukop $\mathrm{T}^{1,4}$, Bartl $\mathrm{M}^{1,5}$, AkovaÖztürk $E^{6}$, Dräger $B^{6}$, Schlotter-Weigel $B^{7}$, Thiele $S^{7}$, Garcia-Angarita $N^{7}$, Greckl $E^{7}$, Dohrn $M F^{8}$, Burkhard $G^{8}$, Reinecke $L^{1}$, Walter $M C^{7}$, Young $P^{9}$, Sereda $\mathrm{MW}^{10}$

Institute 1 Department of Neurology, University Medical Centre Göttingen, Göttingen, Deutschland; 2 University Medical Centre Goettingen, Göttingen, Deutschland; 3 Asklepios Fachklinikum, Psychiatrie, Tiefenbrunn,

Deutschland; 4 Department of Neurogenetics, Max-Planck-Institute for Experimental Medicine, Göttingen, Deutschland; 5 Grünenthal GmbH, Aachen, Deutschland; 6 University Hospital Muenster, Münster,

Deutschland; 7 Ludwig-Maximilians University Munich, München,

Deutschland; 8 AWTH Aachen University, Aachen, Deutschland; 9 Medical

Park Bad Feilnbach Reithofpark, Bad Feilnbach, Deutschland; 10 Department of Neurology, University Medial Centre Göttingen, Göttingen, Deutschland DOI 10.1055/s-0041-1723867

CMT1A is the most common form of hereditary neuropathy. No treatment is available. 164 CMT1A-patients were recruited over 3 years (Göttingen, Munich, Münster and Aachen). Clinical examinations comprised 16 examination scores. Results (change over two years) CMTNSv2 (16,538 to 16,$904 ; \mathrm{p}=0,0077$; $2,2 \%$ ), CMTES ( 11,415 to 11,$794 ; \mathrm{p}=0,0226 ; 3,1 \%)$ and the CMTNSv2-Rash ( 20,463 to 21,$007 ; p=0,0033 ; 2,7 \%$ change) showed a significant increase. Interestingly, only the moderately affected patients showed a significant increase of the CMTNSv2 (from 14,490 to 15,352; $p=0,0481$ ). The ONLS exhibited a highly significant increase $(p=0,0086)$, as well as the 9-HPT $(p=0,0073)$ and the 10-MWT measuring time $(p=0,0271$ ) and steps (from 15,403 to 15,$485 ; p=0,0267$ ). The FSS showed a significant increase from 4,576 up to $5,103(p=0,0006)$. The other parameters did not detect significant changes The compound muscle action potential (CMAP) exhibited a decrease from 4,385 to $3,259(p<0,0001)$ The CMTNSv2 showed a strong positive correlation with the ONLS $(r=0,71)$, the 9-HPT $(r=0,5)$, the 10-MWT $(r=0,48)$, the Walk12 $(r=0,53)$ and the FSS $(r=0,49)$. Discussion In this first longitudinal multicenter national history study of CMT1A patients in Germany, the CMTNSv2, CMTES and CMTNSv2-Rash CMT examination scores were able to detect a significant progression of disease. It is also the first longitudinal trial that examined multiple non-CMT neuropathy scales in CMT1A patients.

\section{P 83 The wide spectrum of symptoms in female XL- MTM carriers - results of an international questionnaire study}

Authors Braun $\mathrm{F}^{1}$, Reumers $\mathrm{SFl}^{2}$, Bonnemann $\mathrm{C}^{3}$, Böhm J ${ }^{4}$, Kamsteeg EJ $\mathrm{J}^{5}$, Schouten $\mathrm{M}^{6}$, Pennings $\mathrm{M}^{5}$, Erasmus $\mathrm{C}^{2}$, Schara-Schmidt $\mathrm{U}^{1}$, Jungbluth $\mathrm{H}^{7}$, Voermans $\mathrm{NC}^{2}$

Institute 1 Department of Pediatric Neurology, Developmental Neurology and Social Pediatrics, University Hospital Essen, Essen, Deutschland; 2 Department of Neurology, Donders Institute for Brain, Cognition and Behaviour, Radboudumc, Nijmegen, Niederlande; 3 National Institute of Neurological Disorders and Stroke, National Institutes of Health, Neuromuscular and Neurogenetic Disorders of Childhood Section, Bethesda, MD, Vereinigte Staaten; 4 Department of Neurobiology and Genetics, Institut de Génétique et de Biologie Moléculaire et Cellulaire, Illkirch, Frankreich; 5 Department of Human Genetics, Radboud University Medical Center, Nijmegen,

Niederlande; 6 Department of Clinical Genetics, Radboud University Medical Center, Nijmegen, Niederlande; 7 Department of Paediatric Neurology, Neuromuscular Service, Evelina's Children Hospital, Guy's \& St. Thomas' Hospital NHS Foundation Trust, London, Vereinigtes Königreich DOI $10.1055 / \mathrm{s}-0041-1723868$ 
Female carriers of X-linked myotubular myopathy (XL-MTM) are often assumed to be asymptomatic. We characterized the spectrum of symptoms in a nonselected cohort of carriers, including prevalence, genetic features, and associated burden in daily life.

We performed an international, cross-sectional online questionnaire study, using questions on neuromuscular symptoms and medical history, the Checklist Individual Strength (CIS), the Frenchay Activities Index, the SF-12 Health Survey (SF-12) and the McGill Pain Questionnaire. We classified carriers as manifesting or non-manifesting, based on self-reported ambulatory status and muscle weakness.

The prevalence of manifesting carriers in this study population $(n=68)$ was $53 \%$, subdivided into mild (43\%), moderate (7\%) and severe (3\%) phenotypes. Next to muscle weakness, manifesting carriers frequently reported fatigue (69\%), exercise intolerance (47\%), muscle cramps (42\%) and myalgia (42\%). Compared to non-manifesting carriers, they scored higher on overall CIS $(p=0.002)$ and fatigue sub-scale $(p<0.001)$. They also scored lower on the physical component of the SF-12 ( $p<0.001)$.

Prevalence of manifesting female XL-MTM carriers is higher than currently assumed, most having a mild phenotype. There is a variety of symptoms, carriers being affected by fatigue, exercise intolerance, limitations in daily activities, reduced physical quality of life and pain. Our findings provide useful information for health care services and future trials.

\section{P 84 Medication in Amyotrophic Lateral Sclerosis - Results from the ALS Registry Swabia}

Authors Rosenbohm $A^{1}$, Braun $A^{2}$, Peter $R^{2}$, Feike $G^{2}$, Kraft I ${ }^{1}$, Lämmle $N^{1}$, Dobias $\mathrm{I}^{1}$, Rothenbacher $\mathrm{D}^{2}$, Dorst $\mathrm{J}^{1}$, Nagel $\mathrm{G}^{2}$, Ludolph $A C^{1}$

Institute 1 Universität Ulm, Neurologie, Ulm, Deutschland; 2 Universität Ulm, Epidemiologie und Medizinische Biometrie, Ulm, Deutschland

DOI 10.1055/s-0041-1723869

Introduction ALS onset and mortality are associated with metabolic factors and potentially by medication. Associations between medications and ALS (risk and prognosis) were evaluated in a population-based setting.

Methods Data from a case-control study embedded in the ALS Registry Swabia of 394 cases and 790 controls was evaluated. Medication at study entry was analyzed for several medication subgroups. Odds ratios (OR) for risk of ALS by medication were modeled using conditional logistic regression and for mortality with adjusted Cox proportional hazard models.

Results Statins were negatively associated with ALS risk (OR=0.39, $95 \%-\mathrm{Cl}$ 0.25 ; 0.59 ) while proton pump inhibitors (PPI) were positively associated $(\mathrm{OR}=1.51,95 \%-\mathrm{Cl} 1.04 ;$ 2.19).Anticonvulsants, antidepressants, Parkinson medication, and antipsychotics showed robust positive associations with ALS. During a median follow-up of 66.5 months, we found a positive association with mortality for ASS ( $H R=1.38,95 \%-\mathrm{Cl} 1.02 ; 1.87)$ and insulin $(H R=2.67$, $95 \%-\mathrm{Cl} 1.17 ; 6.09)$. Thyroxin was negatively associated with mortality ( $\mathrm{HR}=0.70,95 \%-\mathrm{Cl} 0.50 ; 0.97)$.

Conclusion We found a negative association with ALS for statins, and a positive association for PPI use at study entry. Associations of anticonvulsants, antidepressants, Parkinson medication, and antipsychotics mirror treatment of ALS symptoms or comorbidities. Our data support the assumption of lower lipid levels as protective factor in ALS with decreased statin use. 


\section{Namenverzeichnis / Authors' Index}

Abicht A P 61
Admard J P 46
Akova-Öztürk E P 20, P 82
Al Chalabi A V 4
Al Safatli D P 9
Alhaddad B P 46
Allendörfer J P 59
Amsel D P 54
Annoussamy M P 36
Ardicli D P 63
Arendt C P 62
Arlt A V 41, V 48
Aust E P 28
Axer H P 6, P 75

B

Bachtell N P 70

Bajors L P 54

Balousha G P 46

Balousha O P 46

Barresi R P 45

Bartl M P 76, P 82

Baum P P 11, P 12, P 40

Baumann M P 29

Becker LL V 60

Bekele B V 54

Berg B P 69

Beyer I V 25

Bhola PT P 50

Binz C P 10

Biskup S P 81

Blaschek A P 78, P 79

Boentert M P 30, P 31, V 10, V 26

Böhm J P 83

Böhne L P 12

Bonifacino J S P 63

Bonnemann C P 83

Borell S P 23

Brand T V 42

Brandes G P 10

Brandl U P 9

Braun A P 84

Braun F P 36, P 83

Brigadski T P 24

Brill MS P 6, P 75, V 9

Brunkhorst R P 56

Brunn A P 49, P 61

Büchner B P 31

Burkhard G P 82

Burmester GR P 2

Bury G V 48

Busch A P 69

C

Carstens PO P 21

Casteleyn V P 2

Cengiz D P 4, V 7

Chao K P 45

Chio A V 43

Church K P 41

Cirak S P 49, P 50, P 63

Claßen J P 32

Claudi K P 19

Claus I P 64

Conner E P 70

Consortium IN P 81

Cordts I P 30, P 31, P 35

Cortese A P 81

Czech A V 41
D

Dabrowska-Schlepp P P 69

Dafotakis M V 36

Daimagüler H P 49, P 50, P 63

Day JW P 70

DCarvalho M V 11

de Vries H P 9

Deckert M P 49

Della Marina A V 41, V 48

Depienne C V 41

Deschauer M P 30, P 31, P 46

Dettmeyer R P 5

DFrancescantonio S P 71

Diecke S P 73

Diofano F P 49

Dittmayer C P 2, V 38

Dobias I P 84

Dohrn MF P 15, P 56, P 77, P 81, P 82, V 36

Donandt T V 33

Dordevic M P 24, P 8

Dorst ] P 30, P 31, P 35, P 84, V 44

Dötsch J P 49

Dräger B P 20, P 82

Duff J $P 45$

Dumke C P 77

Dziewas R P 64

$\mathrm{E}$

Ebert AK V 54

Eckenweiler M P 27

Eigler V V 36

Emmer A P 14

England E P 45

Erasmus C P 83

Escobar Fernandez H P 48, P 71, P 73, P 74

Esser P P 32

Falna M P 46

Fangerau $\mathrm{H} \vee 1$

Feike G P 84

Fonteyne LM P 68, V 33

Frahm J P 21

Freier E V 41

Friedrich A P 10

Gangfuß A P 36, V 41, V 48

Garcia-Angarita N P 20, P 82

Gaudlitz M P 11, P 40

Gaur N P 18, P 6, P 75

Gazou A P 46

Gazzerro E P 13

Gerriets T P 59

Gess B P 77

Gezer D P 56

Giesert F P 68

Gläser D V 45

Glaubitz S P 1, P 60

Goebel HH P 2, V 38

Gonzales M V 42

Gotthardt M V 29

Götze R P 1

Greckl E P 20, P 80, P 82

Grehl T P 11, P 40, V 27

Griffin D P 41

Grimm A P 15, V 18

Grimmel M P 46

Grond M V 36

Groß, M P 33
Gross CC V 10

Grosskreutz J P 11, P 18, P 35, P 40, P 6, P 75, V 68

Gschwendtberger T P 10

Guglieri M P 42

Günther K P 35

Günther R P 28, P 30, P 31, P 35, P 40

Gutschmidt K V 28, V 45

Haack T P 46

Haastert-Talini K P 10

Hagenacker T P 30, P 31, P 35, V 64

Hahn A P 19, V 42

Hasseli R P 59, P 7

Hathway J P 44

Heidemann M P 80

Heidsieck EM V 28

Heimann N P 59

Heinrich F P 30, P 31

Hengel H P 46

Hentschel A V 41, V 48

Hermann A P 28, P 30, P 31, P 35, V 8

Hermann W P 7

Herty M V 10

Herze R P 13

Hiebeler M P 39

Hintze S P 47, P 52, P 69

Hirsch I P 76

Hofer S P 21

Hornemann T P 77

Huang Z P 10

Hudson J P 45

Husain RA P 9

!

lammarino M P 41

Inashkina I P 45

Jensen I P 44

Jordan B P 46, V 39

Jungbluth H P 83

Just S P 49

K

Kaindl A V 60

Kalbe C V 33

Kalms N P 7

Kamsteeg EJ P 83

Kao SY P 47

Karaca E P 50

Kasper D P 51

Kassubek J P 16, P 55, V 19

Katona I V 36

Kendzierski T P 14, V 35, V 39, V 40

Keren-Kaplan T P 63

Kernohan KD P 50

Kessler B $\vee 33$

Kettemann D P 11, P 40

Keuper M P 5

Khan N P 42

Kieshauer J V 54

Kirschke J P 46

Kirschner J P 23, P 27, V 56

Klaus B P 24, P 8

Klawonn F V 55

Kleeberg K P 14

Klein O V 63

Klopstock T P 30, P 31

Knoepffler N V 2 
Koс Y P 11, P 40

Kocatürk NM P 49

Koch B P 35

Koch JC P 30, P 31, P 35, P 40

Koenig E P 42

Koester F P 17

Kohl Z P 30, P 31, P 35

Kohlschmidt N V 41, V 48

Kölbel H P 23, P 78, P 79, V 41, V 48

Kornblum C P 26, P 46, V 34, V 53

Kostera-Pruszczyk A P 50

Kraft I P 84

Krahn AB P 76, P 82

Krämer HH P 7

Krause A P 74

Krause S P 37, P 39, P 68, P 80, V 33

Kraya T P 17, P 46, V 39

Kreiß M P 46

Kress W P 53

Krück S P 5

Kühn R P 48

Kummer K P 1, P 25, P 34, P 60

Kupatt C P 68

Kurth I P 77

Labeit B P 38, P 64

Lace B P 45

Lämmle N P 84

Langer T P 27

Leha A P 20, P 82

Lehman K P 41

Lehmann H P 61

Lessmann V P 24

Lewis S P 41

Liebmann-Reidl S P 51

Lingor P P 30, P 31, P 35

Linhoff L P 20

Linker R P 30, P 31

Linse K P 28

Löscher WN P 35

Lotz J P 21

Lowes L P 41

Ludolph AC P 16, P 19, P 35, P 55, P 84, V 19

Lukas K P 58

Lupski JR P 50

M

MacArthur D P 45

Maier A P 11, P 35, V 14

Marg A P 71, P 74, V 54

Maron E P 13

Martakis K P 19

Marten A V 10

Matthes C P 19

Mayer TE P 18

Meadows A P 41

Mechtler T P 51

Mehlhorn C P 9

Mehnert-Theuerkauf A P 32

Meier A P 40

Meinke P P 47, P 52, P 69

Meisel A V 5

Meitinger T P 46

Mendell J P 41

Mensch A P 14, P 17, V 39, V 40

Mercuri E P 42

Metelmann M P 11, P 30, P 31, P 32, P 40

Metzler E P 73

Metzner K P 6, P 75

Meuth SG P 4, V 10, V 7

Meyer S P 25

Meyer T P 11, P 35, P 40, V 58

Micheilis T P 34

Michels S P 35
Miller N P 41

Mohamed M V 19

Montagnese F V 37, V 45

Moretti A P 68

Mozaffar T P 70

Mroczek M P 45

Mueller-York A P 41, P 42

Muhle P P 64

Müller C P 27

Müller CK P 23

Müller HP P 16

Müller N P 24, P 8

Müller P P 24, P 8

Müller T P 17

Müller-Felber W P 78, P 79, V 57

Müller-Ladner U P 59, P 7

Münch C P 11, P 40

Müntefering T P 4, V 7

Muntoni F P 42

Müthel S P 74, V 32

N

Naegel S P 17, V 39

Nagel G P 84

Nägel S P 14, V 35, V 40

Nagels KH P 37

Nanda I P 53, V 51

Nelke C P 22, P 38, P 4, V 7

Neubauer BA P 19

Neuwirth C V 12

Nguyen C V 48

Nikolin S P 56, P 77, V 36

Nikonova E V 31

Nishino I V 37

Norden J P 11, P 40

Nürnberg P P 50, P 63

0

Obermaier CD P 81

Ohlendieck K V 47

Olthoff A P 21

Osmanovic A P 30, P 31

Otto M P 19, V 21

Ouwenbroek A P 77

Özdemir Ö P 50

Pauli S P 25, P 60

Pawlitzki M P 22, P 38

P 64, V 10

Pechmann A P 23, P 27

Penka D P 7

Pennings M P 83

Pergande M P 50

Perlova K P 64

Peseschkian T P 30, P 31

Peter R P 84

Petri S P 10, P 11, P 30, P 31 ,

P 35, V 49

Peuker M P 32

Pfeuffer S V 10

Pickrodt K P 25

Pluta N V 51

Pohlner J P 3

Posey JE P 50

Pozsgai E P 41

Prell T P 18

Preuße C P 2

Prudlo J P 35, V 50

Prukop T P 20, P 76, P 82

Punetha J P 50

$$
\text { R }
$$

Rabenstein M P 61

Rauch A P 50
Rebel M V 36

Rebelo A P 81

Regensburger M P 30, P 31

Reichart R P 9

Reichert S P 68

Reilich P P 39, P 80

Reimann J P 26, V 34

Reinecke L P 20, P 76, P 82

Reumers SF. I P 83

Richert C V 63

Riess O P 46

Rödiger A P 6, P 75

Rodino-Klapac L P 41

Roediger A P 18

Rolfes L P 38, V 10

Rolke R P 77

Roos A P 36, V 41, V 48

Rosenbohm A P 55, P 84, V 19

Rosenfelder S P 23

Rossner MJ P 20

Rost S P 53, V 51

Rothenbacher D P 84

Ruck T P 22, P 38, P 4, P 59, P 64, V 7

Rückoldt J P 10

Rudnik-Schöneborn S P 29

Ruetz A P 67

Rupp S P 5

Rupprich K P 46

Saak A P 66, V 69

Saffari A V 22

Salimi Dafsari H P 49

Sarikidi A P 35

Sauer S P 73

Schaaf A P 69

Schaefer J P 66, V 69

Schänzer A P 5, P 54, P 59, P 7, V 42

Schara-Schmidt U P 23, P 36, P 46, P 78,

P 79, P 83, V 41, V 48, V 62

Schischlevskij P P 30, P 31

Schlotter-Weigel B P 20, P 46, P 82

Schmicker M P 24, P 8

Schmidt G P 5

Schmidt J P 1, P 21, P 25, P 3, P 37, P 60, V 6

Schmidt K P 37

Schmitt D P 36, P 44

Schneider I P 30, P 31, P 46, V 39, V 63

Schneider U P 2

Schöls L P 46

Schöne U P 56

Schorling DC P 23, P 27

Schoser B P 47, P 52, P 58, P 65, P 69, P 70, V 28 ,

V 37, V 45, V 52

Schouten M P 83

Schöwel-Wolf V V 54

Schreiber S P 10, P 24, P 8, V 10

Schreiber-Katz O P 11, P 30, P 31

Schröder R V 36

Schröter C P 12, P 30, P 31, V 24

Schubert I V 7

Schulte-Mecklenbeck A V 10

Schulz JB P 56, P 77

Schütz S P 21

Schwartz O P 78, P 79

Schwarz M P 51

Sehring J P 5, P 54

Seif A P 21

Senft C P 9

Senn KC P 37

Sereda MW P 20, P 76, P 82

Servais L P 36, P 42

Shukla A P 50

Shy M P 81

Sickmann A V 41, V 46, V 48 
Siegert E P 2

Slocomb T P 44

Sobalska-Kwapis M P 45

Sonja F P 20, P 76, P 82

Sperfeld AD P 11

Spittel S P 11, P 35, P 40

Spletter M P 47, V 31

Sprute R P 50

Spuler S P 13, P 48, P 71, P 73,

P 74, V 32, V 54

Stadelmann C P 71

Stavusis ] P 45

Steinbach R P 18, P 35, P 40, P 6, P 75

Stendel C P 30

Stenzel W P 2, V 38

Stoevesandt D P 17, V 39

Stoltenburg C V 60

Stoltenburg-Didinger G P 14, V 35,

V 39, V 40, V 63

Strapagiel D P 45

Straub V P 42, P 45, V 61

Streubel B P 51

Strom T P 46

Stroth I P 39

Sturm M P 46

Sunaga-Franze DY P 73

Suntrup-Krueger S P 64

Thau-Habermann N P 10

Thiele S P 20, P 37, P 39, P 80, P 82

Todorow V P 47

Topaloglu H P 63

Töpf A P 45

Tschernatsch M P 59

Tumani H P 19

Turner M V 20
Uecker M P 21

Urban D V 19

Uruha A P 2

$\mathrm{V}$

Van den Berg L V 59

van der Stam L V 60

van Wickeren N P 24, P 8

Veldink JH V 67

Vereb N V 45

Vielhaber S P 24, P 8, V 10, V 17

Vill K P 78, P 79

Vittore D P 15

Voermans NC P 83

von Landenberg C P 26, V 34

von Moers A V 38, V 51

Vorgerd M P 46

Vozobulova M P 75

W

Walter B P 11, P 40

Walter MC P 20, P 37, P 39, P 68 ,

P 80, P 82, V 30, V 33

Wang $\mathrm{H} \mathrm{P} 74$

Wang S P 42

Warnecke T P 64

Wassner A P 55

Weidenmüller M P 21

Weiß C V 60

Weis J P 46, P 49, P 56, V 36, V 39, V 41

Wenninger S P 58, P 70, V 28

Weydt P P 11, P 40, V 15

Weyen U P 11, P 30, P 31, P 40

Wiegand-Grefe S V 3

Wiendl H V 10

Wiesinger T P 51
Wigge S P 76

Winkler M P 26, V 34

Winter N P 15

Wirner C P 12, V 28

Wisch H P 19

Witte OW P 18

Witzel S P 35

Wolf E P 68, V 33

WolfJ P 30, P 31, P 35, P 40

Wong PFoo C P 70

Wunderlich G P 61

Wurst W P 68

Wurster C P 19

Y

Young P P 20, P 82, V 23

Zaum AK P 53, V 51

Zechel S P 1, P 25, P 60

Zeller D P 30, P 31

Zeng R P 21

Zhogov A P 48

Zieger B P 23

Ziegler A V 22, V 65

Zierz S P 14, P 17, P 46, P 62, V 35 ,

$\vee 39, \vee 40, \vee 63$

Zimmer F V 51

Zschüntzsch J P 25, P 3, P 34

Züchner SL P 81

Zunk L P 54 